\title{
Optimality in Human Motor Performance: Ideal Control of Rapid Aimed Movements
}

\author{
David E. Meyer \\ University of Michigan \\ Sylvan Kornblum \\ University of Michigan
}

\author{
Richard A. Abrams \\ Washington University \\ Charles E. Wright \\ Columbia University
}

\author{
J. E. Keith Smith \\ University of Michigan
}

\begin{abstract}
A stochastic optimized-submovement model is proposed for Fitts' law, the classic logarithmic tradeoff between the duration and spatial precision of rapid aimed movements. According to the model, an aimed movement toward a specified target region involves a primary submovement and an optional secondary corrective submovement. The submovements are assumed to be programmed such that they minimize average total movement time while maintaining a high frequency of target hits. The programming process achieves this minimization by optimally adjusting the average magnitudes and durations of noisy neuromotor force pulses used to generate the submovements. Numerous results from the literature on human motor performance may be explained in these terms. Two new experiments on rapid wrist rotations yield additional support for the stochastic optimizedsubmovement model. Experiment 1 revealed that the mean durations of primary submovements and of secondary submovements, not just average total movement times, conform to a square-root approximation of Fitts' law derived from the model. Also, the spatial endpoints of primary submovements have standard deviations that increase linearly with average primary-submovement velocity, and the average primary-submovement velocity influences the relative frequencies of secondary submovements, as predicted by the model. During Experiment 2, these results were replicated and extended under conditions in which subjects made movements without concurrent visual feedback. This replication suggests that submovement optimization may be a pervasive property of movement production. The present conceptual framework provides insights into principles of motor performance, and it links the study of physical action to research on sensation, perception, and cognition, where psychologists have been concerned for some time about the degree to which mental processes incorporate rational and normative rules.
\end{abstract}

An enduring issue in the study of the human mind concerns the rationality and optimality of the mental processes that guide thought and action. Philosophers and psychologists have long debated about the extent to which people's reasoning and problem solving adhere to the rules of formal logic (e.g., see Braine, 1978; Holland, Holyoak, Nisbett, \& Thagard, 1986; Osherson, 1975). Similarly, there has been much interest in how well decision making and choice behavior adhere to the normative rules

Portions of this research were first presented at a meeting of the Psychonomic Society (Abrams, Kornblum, Meyer, \& Wright, 1983). Funding was provided by National Institute of Mental Health Grant R01 MH37145 to the University of Michigan, Sylvan Kornblum and David E. Meyer, principal investigators. Richard A. Abrams participated under a traineeship from the National Institutes of Health (Grant T32 EY07022) and a fellowship from the Horace Rackham School of Graduate Studies at the University of Michigan.

We thank S. Keele, D. Rosenbaum, and R. Schmidt for helpful comments. Carol Huff and Peggy Kwun provided superb technical assistance.

Correspondence concerning this article should be addressed to David E. Meyer, Human Performance Center, Department of Psychology, University of Michigan, 330 Packard Road, Ann Arbor, Michigan 48104. of mathematical probability theory and statistical decision theory (e.g., see Edwards, 1961; Edwards, Lindman, \& Savage, 1963; Holland et al., 1986; Kahneman \& Tversky, 1973; Nisbett \& Ross, 1980; Tversky \& Kahneman, 1974, 1981). It is generally agreed now that without explicit training, people do not always behave exactly as formal logic, probability theory, or decision theory would prescribe. Nevertheless, their behavior may approximate the ideal, subject to limitations in memory capacity and information-processing resources (Simon, 1957).

The present article extends this concern with rationality and optimality to the domain of human motor performance. We focus especially on the production of rapid aimed movements in which a part of the body (e.g., finger, arm, leg, head, eye, tongue) must be shifted quickly and accurately from some initial position to another desired terminal position. Such movements merit careful scrutiny because they constitute the basic building blocks of numerous everyday activities (e.g., pointing, reaching, touching, grasping, walking, looking, talking) and because they contribute to many complex physical skills. As for higher mental processes, our goal is to demonstrate how rapid aimed movements may be understood on the basis of ideal operating characteristics that are conditioned by inherent limitations in system capacities and resources. Moreover, through this demonstra- 


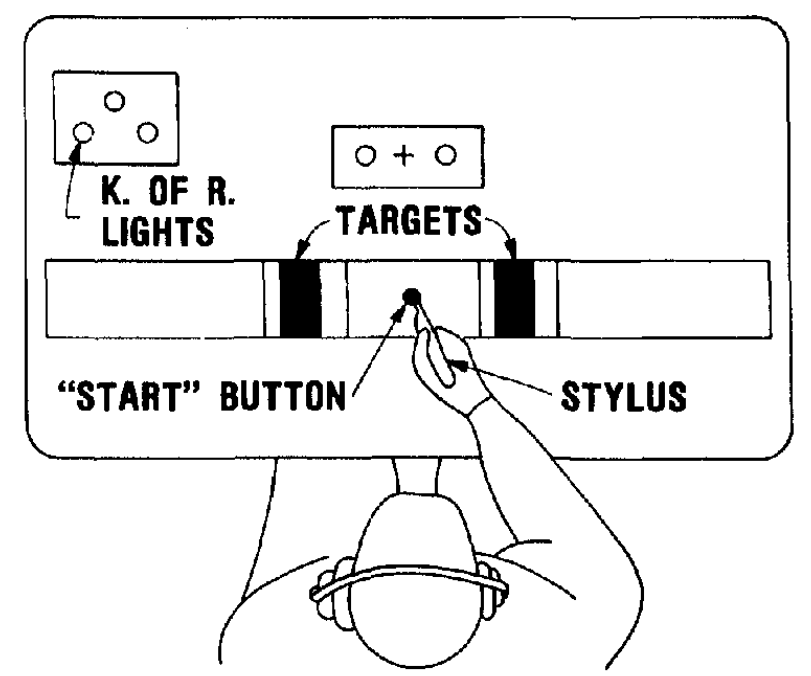

Figure 1. The stylus-tapping paradigm in which Fitts' law was first demonstrated for rapid spatially constrained movements. (From "Information Capacity of Discrete Motor Responses" by P. M. Fitts and J. R. Peterson, Journal of Experimental Psychology, 1964, 67, 103-112. Copyright 1964 by the American Psychological Association. Reprinted with permission of publisher.)

tion, we hope to strengthen the ties between cognitive psychology and motor performance.

\section{Logarithmic Speed-Accuracy Trade-off}

The starting point of our exposition is a classic article by Fitts (1954) on human motor performance. In Fitts' (1954) work, a logarithmic trade-off was discovered between the duration and spatial precision of rapid aimed limb movements. The paradigm for this discovery appears in Figure 1. Fitts had subjects move a hand-held stylus back and forth between two target regions. The subjects were supposed to hit the targets with high frequency (e.g., making approximately $95 \%$ of their movements end inside the targets), while minimizing their average (mean) movement times. ${ }^{1}$ Across different experimental conditions, the width $(W)$ of the target regions and the distance $(D)$ between the target centers were varied systematically. The average movement time $(T)$ closely approximated an equation of the form

$$
T=A+B \log _{2}(2 D / W),
$$

where $A$ and $B$ are positive constants, and $\log _{2}(2 D / W)$ is a measure of spatial precision scaled in terms of information bits. Fitts (1954) referred to $\log _{2}(2 D / W)$ as the index of difficulty (ID) of an aimed movement. ${ }^{2}$

Equation 1 has become known as Fitts' law. Numerous studies have shown it to hold for a variety of movements produced under different environmental conditions. The logarithmic speed-accuracy trade-off applies not only to stylus tapping but also to wrist flexion and rotation (Crossman \& Goodeve, 1963/ 1983; Jagacinski, Repperger, Moran, Ward, \& Glass, 1980), arm extention (B. A. Kerr \& Langolf, 1977), and finger manipulation (Langolf, Chaffin, \& Foulke, 1976). Similar results regarding Fitts' law have been obtained in laboratory settings (Annett, Golby, \& Kay, 1958; Fitts \& Peterson, 1964) and real- world situations (e.g., under water; R. Kerr, 1973). Both normal subjects and abnormal individuals such as mental retardates (Wade, Newell, \& Wallace, 1978) and patients with Parkinson's disease (Flowers, 1976) exhibit the same general form of tradeoff function. In light of the broad generality of these results, Fitts' law appears to reflect a fundamental property of human motor performance, and consequently, researchers in areas such as human-factors engineering (Bailey \& Presgrave, 1958; Barnes, 1963) and neuropsychology (Flowers, 1976) have been strongly influenced by Fitts' (1954) original report.

\section{Controversies Over Fitts' Law}

Yet studies regarding Fitts' law have not proceeded entirely without controversy. Some investigators have proposed different logarithmic speed-accuracy trade-off functions to characterize rapid aimed movements (e.g., Crossman \& Goodeve, 1963/1983; Sheridan, 1979; Welford, 1968), and others have suggested that either power or linear functions are more appropriate under certain circumstances (e.g., Howarth, Beggs, \& Bowden, 1971; Kvalseth, 1980; Meyer, Smith, \& Wright, 1982; Schmidt, Zelaznik, \& Frank, 1978; Schmidt, Zelaznik, Hawkins, Frank, \& Quinn, 1979). The exact form of the obtained trade-off may depend on specific task demands. For example, Wright and Meyer (1983) have drawn a distinction between tasks involving spatially constrained movements and tasks involving temporally constrained movements (cf. Fitts, 1954). Spatially constrained movement tasks are ones in which subjects must terminate their movements within a specified target region while attempting to minimize their average movement times, as required by Fitts' stylus-tapping task. Temporally constrained movement tasks are ones in which subjects must produce movements having precisely specified durations while terminating the movements as close as possible to a discrete target point, not a region (e.g., trying to hit a target point with a movement that lasts exactly $200 \mathrm{~ms}$ ). A linear trade-off function has been found to fit better than a logarithmic function for temporally constrained movements (Schmidt et al., 1978, 1979; Wright, 1983a, 1983b; Wright \& Meyer, 1983; Zelaznik, Shapiro, \& McColsky, 1981), whereas a logarithmic function (Equation 1) fits better for spatially constrained movements. By varying the amount of emphasis placed on spatial versus temporal precision, subjects may be induced to shift from exhibiting one form of trade-off function to exhibiting the other (Zelaznik, Mone, McCabe, \& Thaman, in press). Such flexibility raises fundamental theoretical issues concerning the relation between the alternative trade-offs (cf. Hancock \& Newell, 1985).

\footnotetext{
${ }^{1}$ Following conventional terminology (Keele, 1968), we use the term movement time to designate the duration of the time interval from when a movement begins overtly to when it ends. This term should not be confused with the terms reaction time and movement latency, which designate the duration of the time interval between the onset of a response signal and the beginning of an overt movement.

${ }^{2}$ The index of difficulty represents the number of bits of information required to designate a particular region having width $W$ within a set of $n$ such contiguous regions that evenly partition the interval $\{0,2 D]$. For example, if $D=16$ and $W=4$, then $n=8$ and $I D=3$ (cf. Shannon \& Weaver, 1949).
} 
Controversy has also arisen over what type of model best explains Fitts' law for situations in which the logarithmic speedaccuracy trade-off holds. The theoretical debate can be understood in terms of a proposal made by Woodworth (1899). He suggested that an aimed movement may consist of a programmed, ballistic, initial-impulse phase followed by a currentcontrol phase in which sensory feedback is used to correct unintended errors after the movement has begun (cf. Poulton, 1974; Welford, 1968). Following this suggestion, several investigators have attributed the logarithmic speed-accuracy trade-off (Fitts' law) to the nature of the current-control phase (e.g., Crossman \& Goodeve, 1963/1983; Keele, 1968; Schmidt et al., 1979), whereas others have attributed it to the nature of the initialimpulse phase (e.g., Jagacinski et al., 1980; Langolf et al., 1976; Meyer et al., 1982). Some data support the latter viewpoint over the former, but details of the initial-impulse and current-control phases still need to be explicated definitively.

\section{A New Approach}

The remainder of this article further considers the empirical and theoretical bases of Fitts' law, relating them more closely to the linear speed-accuracy trade-off and other aspects of human motor performance. First, we summarize one major account, the deterministic iterative-corrections model (Crossman \& Goodeve, 1963/1983; Keele, 1968), that has been formulated to rationalize Fitts' law for rapid spatially constrained movements. Several significant deficiencies of this model will be noted.

Next, we introduce an alternative account, the stochastic optimized-submovement model, that shares some features with the deterministic iterative-corrections model but goes beyond it to handle a considerably broader range of data. Explicit connections will be drawn between the stochastic optimized-submovement model and previous work (Meyer et al., 1982; Schmidt et al., 1978, 1979; Wright, 1983a, 1983b; Wright \& Meyer, 1983) on the random variability of noisy neuromotor force pulses used to generate aimed movements. We hypothesize that the production of rapid spatially constrained movements may be characterized in terms of an ideal compromise between the durations of component submovements made under the influence of such noise. It will be shown that this hypothesis explains several previously puzzling results in the motorperformance literature.

Then we report the results of two new experiments conducted to test additional predictions derived from the stochastic optimized-submovement model. These predictions deal with the exact form of the speed-accuracy trade-off for spatially constrained movements and with other kinematic properties observed under various visual-feedback conditions. We will show that a power (viz., square-root) function based on the model may offer as good an approximation to the movement-time data as does a logarithmic function (Equation 1) and that certain characteristics of submovements implied by the model hold regardless of whether or not subjects receive visual feedback during their movements.

Together, our results and model establish a unified conceptual framework from which to pursue future research regarding human motor performance and rapid aimed movements. The notion of stochastic optimized submovements may also lead to connections with research on the optimality of sensation, perception, and cognition.

\section{Deterministic Iterative-Corrections Model}

For about 25 years, the best known explanation of Fitts' law has been the deterministic iterative-corrections model, which was formulated originally by Crossman and Goodeve (1963/ 1983) and elaborated subsequently by Keele (1968) and other investigators (e.g., Meyer et al., 1982). This model assumes that an overall movement from an initial home position to a target region includes a series of discrete submovements made on the basis of sensory feedback. Each submovement supposedly has a well-defined beginning and end, takes a constant time increment $(t)$ to complete, and travels a constant proportion $\left(p_{\mathrm{d}}\right)$ of the remaining distance to the center of the target. ${ }^{3}$ The model is "deterministic" in the sense that it incorporates no random variability due to neuromotor noise or other stochastic factors; the extents and durations of component submovements are assumed to have fixed values across movement trials involving the same combination of target distance and width.

These assumptions are illustrated in Figure 2. Here we have shown an idealized plot of movement velocity versus distance for a series of three hypothetical submovements (solid curves) that start from a home position (distance $=0$ ) and proceed to a target region (distance $=D$, width $=W$ ). According to the deterministic iterative-corrections model, the first, second, and third submovements travel distances of $p_{\mathrm{d}} D, p_{\mathrm{d}} D\left(1-p_{\mathrm{d}}\right)$, and $p_{\mathrm{d}} D\left(1-p_{\mathrm{d}}\right)^{2}$, respectively. Termination of the submovement series occurs as soon as a submovement ends inside the target region (i.e., when the remaining distance to the center of the target is less than $W / 2){ }^{4}$

Either visual or kinesthetic feedback could, in principle, be used to guide the submovements of the deterministic iterativecorrections model (Crossman \& Goodeve, 1963/1983). However, there is considerable evidence that subjects do not produce corrective submovements very effectively on the basis of kinesthetic feedback (Abrams, Kornblum, Meyer, \& Wright, 1983; Carlton, 1981a, 1981b; Prablanc, Echallier, Komilis, \& Jeannerod, 1979; Vince, 1948; Wallace \& Newell, 1983; Woodworth, 1899). So it seems likely that visual feedback may pro-

\footnotetext{
${ }^{3}$ Operationally, a submovement is defined as a change in the position of a body part during a time interval such that (a) at the beginning of the interval, the velocity of the body part crosses a near-zero threshold or certain marked reversals occur in its acceleration, (b) one of these types of events occurs again at the end of the interval, (c) the absolute value of the velocity throughout the interval exceeds a specified criterion, and (d) the total distance traveled from the beginning to the end of the interval exceeds a specified criterion. The criteria for this definition may be selected to discriminate voluntary submovements from passive involuntary activity (e.g., tremor). For a more complete treatment of such matters, see Experiment 1.

${ }^{4}$ If more than three submovements are needed to reach the target, then the submovement series would continue onward in the same fashion; for example, the $n$th submovement would travel a distance of $p_{\mathrm{d}} D\left(1-p_{\mathrm{d}}\right)^{n-1}$. In this expression, the term $D\left(1-p_{\mathrm{d}}\right)^{n-1}$ represents the amount of distance left between the end of the $n-1$ st submovement and the target center. The term $p_{\mathrm{d}}$ is the proportion of this distance that the $n$th submovement would travel.
} 


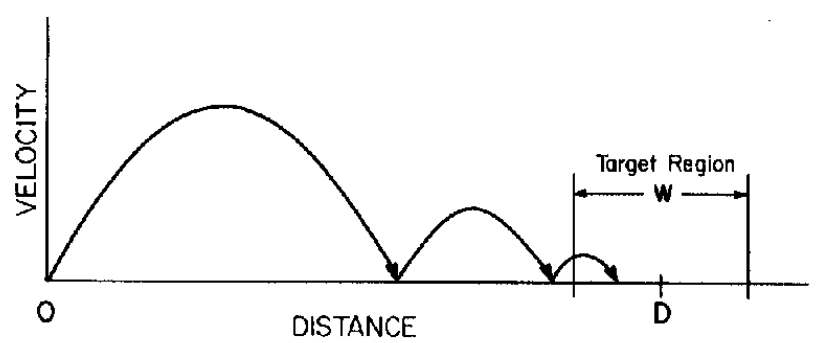

Figure 2. Outline of assumptions for the deterministic iterative-corrections model (Crossman \& Goodeve, 1963/1983; Keele, 1968). (The horizontal axis represents movement distance, and the vertical axis represents movement velocity. The solid curves correspond to successive hypothetical submovements between an initial home position [distance $=$ 0 ] and a target region [bounded by vertical lines] whose center is $D$ units from the home position and whose width is $W$ units.)

vide the main information for making necessary corrections, as assumed under Keele's (1968) version of the model.

\section{Account of Fitts' Law}

Using the deterministic iterative-corrections model, it is possible to account quantitatively for Fitts' law. The account stems directly from the fact that for a specified target region, the number of submovements expected under the model increases in an approximately logarithmic fashion as a function of $D / W$ (Crossman \& Goodeve, 1963/1983; Keele, 1968). ${ }^{5}$ If each submovement takes a constant increment of time, as the model assumes, then the total time taken by the series of submovements would thus be an approximately logarithmic function of $D / W$, yielding Equation 1. In essence, this account attributes Fitts' law to the nature of the current-control phase of an aimed movement.

The deterministic iterative-corrections model is also consistent with some other observations regarding rapid spatially constrained movements. Crossman and Goodeve (1963/1983) found periodic fluctuations in the velocity profiles of wrist rotations to specified target regions, suggesting the occurrence of multiple submovements. Multiple submovements likewise appeared at least occasionally during studies by Carlton (1979, 1980), Langolf et al. (1976, Experiment 1), and Jagacinski et al. (1980). This is what one would expect if feedback-guided iterative corrections mediate the logarithmic speed-accuracy trade-off (Fitts' law). ${ }^{6}$

\section{Limitations of the Model}

Nevertheless, despite the attractive features of the deterministic iterative-corrections model, there are some compelling reasons to question its ultimate viability. Although the model provides a neat quantitative account of Fitts' law, it cannot deal with other observed characteristics of rapid aimed movements, such as the duration of primary (initial) submovements, the spatial variability of submovement endpoints, the relative frequency of higher order (e.g., secondary) corrective submovements, the relative frequency of errors (target misses), and the effects of feedback deprivation. These characteristics, which lie at the heart of the logarithmic speed-accuracy trade-off, are important for any theory of motor performance to explain.

Primary-submovement durations. We will first consider the durations of primary submovements (i.e., ones that initiate a submovement series toward a target region). In studies of spatially constrained movements, a few investigators have reported that target distance or width did not affect primary-submovement durations, even though the average total movement times obeyed Fitts' law (e.g., Annett et al., 1958; Langolf et al., 1976, Experiment 1). This is consistent with the deterministic iterative-corrections model, which assumes constant submovement durations.

Such constancy may, however, be limited to relatively unusual circumstances. For example, Langolf et al. (1976, Experiment 1) found primary-submovement durations to be unaffected by target width only when subjects made extremely small finger movements while receiving visual feedback through a microscope. Under more typical circumstances, investigators have observed marked effects of both target distance and width on the duration of primary submovements (e.g., Jagacinski et al., 1980; Langolf et al., 1976, Experiment 2). As the distance of the target increases or the target width decreases, primary-submovement durations usually increase. This contradicts the deterministic iterative-corrections model.

Submovement-endpoint variability. Another obvious problem for the deterministic iterative-corrections model concerns the inherent spatial variability of submovement endpoints. The model assumes that each submovement always travels a constant proportion of the remaining distance to the center of the target. This assumption plays a major role in the model's account of Fitts' law (see Footnote 5), but it is clearly wrong. Primary submovements as well as subsequent higher order corrective submovements do not travel constant proportional distances. Considerable variability occurs in primary-submovement endpoints even when the target distance and width are fixed (Jagacinski et al., 1980; Langolf et al., 1976). Such variability, which subjects cannot completely eliminate, has been studied mainly in experiments on temporally constrained movements (e.g., Schmidt et al., 1978, 1979; Wright, 1983a, 1983b; Wright \& Meyer, 1983; Zelaznik et al., 1981), while remaining relatively ignored in experiments on spatially constrained movements (cf. Welford, 1968). Yet it could have an important bearing on the source of the logarithmic speed-accuracy trade-off (Schmidt et al., 1978).

\footnotetext{
${ }^{5}$ More specifically, suppose that $n$ is the expected number of submovements. Then $n$ must be the smallest integer such that $\sum_{\mathrm{i}=1} p_{\mathrm{d}} D(1-$ $\left.p_{\mathrm{d}}\right)^{i-1}>D-W / 2$. Consequently, $n=1+\left\lfloor\log _{2}(2 D / W) / \log _{2}\left(1-p_{\mathrm{d}}\right)^{-1}\right\rfloor$, where the floor symbols $(\mathrm{L}\rfloor)$ denote the greatest integer less than or equal to the enclosed ratio of the two logarithms (Keele, 1968).

${ }^{6}$ On the basis of reports by Vince (1948) and by Keele and Posner (1968), Keele (1968) estimated that each submovement travels about $93 \%$ of the remaining distance to the center of the target (i.e., $p_{\mathrm{d}}=.93$ ) and that the duration of each submovement equals about $200 \mathrm{~ms}$. These estimates allowed him to predict the slope of the logarithmic trade-off function (i.e., the constant $B$ in Equation 1) with good accuracy. His predictions, like some available data (e.g., Fitts, 1954), placed this slope at about $70 \mathrm{~ms}$ per unit of $I D$ (i.e., $\log _{2}(2 D / W)$ ) for aimed stylus-tapping movements.
} 
Relative frequencies of secondary submovements. Because of its failure to deal with submovement-endpoint variability, the deterministic iterative-corrections model also has trouble explaining the observed relative frequencies of secondary submovements, which sometimes follow primary submovements. For any ratio of target distance to width, the model predicts that secondary submovements should always or never occur, depending on the value of $D / W$ and the assumed constant proportion $\left(p_{\mathrm{d}}\right)$ of distance traveled by the primary submovements. There should always be a secondary submovement if $D / W \geq$ $.5\left(1-p_{\mathrm{d}}\right)^{-1}$, because then the primary submovement would never be long enough to end inside the target region. There should never be a secondary submovement if $D / W<.5(1-$ $\left.p_{\mathrm{d}}\right)^{-1}$, because then the primary submovement would always be long enough to end inside the target region. This prediction is violated by the finding that secondary submovements occur probabilistically with relative frequencies whose magnitudes increase gradually from zero to one as a function of $D / W$ (e.g., Carlton, 1979, 1980; Jagacinski et al., 1980).

In some cases, investigators have also examined the trajectories of spatially constrained movements without finding much, if any, evidence of multiple submovements across various target distances and widths. For example, the results of Langolf et al. (1976, Experiment 2) and Jagacinski et al. (1980) revealed numerous movement trajectories that were essentially continuous and did not contain multiple submovements, but the associated movement times still approximated a logarithmic speed-accuracy trade-off. The deterministic iterative-corrections model cannot accommodate this latter outcome, because the model's account of Fitts' law rests entirely on variations in the number of submovements expected as a function of $D / W$ (Footnote 5 ; cf. Legge \& Barber, 1976). ${ }^{7}$

Movement errors. The deterministic iterative-corrections model likewise fails to account for errors (target misses) that occur in the production of rapid spatially constrained movements. Because of inherent random variability, a series of submovements may not always end inside a specified target region. Instead, the error rate tends to increase as the target distance increases or width decreases (Fitts, 1954; Fitts \& Peterson, 1964; Wallace \& Newell, 1983). On the other hand, the deterministic iterative-corrections model assumes that subjects always continue making corrective submovements until they have reached the target. This assumption is what allows the model to yield a logarithmic trade-off function. Consequently, if the model were correct, virtually no errors should occur during motor performance, contrary to available data.

Effects of feedback deprivation. Finally, it is important to consider the effects of feedback deprivation on rapid spatially constrained movements. In some experiments on Fitts' law, visual feedback has been eliminated during the progress of movements toward specified target regions, and performance has been compared with results obtained under conditions of full feedback. Investigators have found that visual-feedback deprivation reduces movement accuracy (i.e., target hits) significantly. They have reported, however, that average movement times still vary logarithmically with $D / W$ (Abrams et al., 1983; Prablanc et al., 1979; Wallace \& Newell, 1983). Fitts' law may also hold for movements whose durations are sufficiently short to preclude visual feedback from being used to correct them. As Schmidt et al. (1979) have pointed out, such results raise additional doubts about the rationale for the deterministic iterative-corrections model, which depends heavily on the availability and use of visual feedback (Keele, 1968).

\section{Stochastic Optimized-Submovement Model}

Given the preceding empirical and theoretical considerations, we have developed an alternative account for the production of rapid spatially constrained movements. This account involves a stochastic optimized-submovement model that stems from work on impulse variability in temporally constrained movements (Meyer et al., 1982; Schmidt et al., 1978, 1979; Wright, 1983a, 1983b; Wright \& Meyer, 1983). Our approach modifies some assumptions of the classical deterministic iterative-corrections model (Crossman \& Goodeve, 1963/1983; Keele, 1968) and replaces other assumptions with more plausible ones. A key feature of the present conceptual framework is that it represents the movement-production process as an ideal compromise between the durations of primary and secondary submovements. The form of the compromise depends on assumptions about neuromotor noise and the spatial variability of submovement endpoints. Using these assumptions and the hypothesis that submovement durations are appropriately optimized to cope with the noise, we can reconcile a number of phenomena not handled by earlier accounts. Moreover, the present framework builds a solid bridge between Fitts' law and the linear speed-accuracy trade-off observed in studies of temporally constrained movements.

\section{Basic Assumptions}

An outline of the stochastic optimized-submovement model appears in Figure 3. The figure shows hypothetical trajectories (velocity vs. distance) for three trials on which rapid aimed movements are made from an initial home position to a specified target region. Each of the trajectories includes a primary submovement (solid curves), and two of them also include secondary corrective submovements (dashed curves). Regarding this representative situation, the model incorporates six basic assumptions. The assumptions concern (a) the existence of noise in the neuromotor system, (b) the number of submovements produced to reach the target region, (c) the distributions of submovement endpoints in space, (d) the effect of neuromotor noise on the variances of these distributions, (e) the minimization of movement times with respect to the noise, and (f) the processing of various types of information for movement preparation and execution.

Neuromotor noise. Under the stochastic optimized-submove-

\footnotetext{
${ }^{7}$ According to Vince (1948) and Carlton (1979), a primary submovement typically travels $90 \%$ or more of the distance from its starting location to the center of the target. Thus, if each subsequent corrective submovement travels an equally large proportion (i.e., $p_{\mathrm{d}} \geq .9$ ) of whatever distance remains at the end of the immediately preceding submovement, then the target region should always be reached within two submovements except when $D / W$ is extremely large (e.g., $D / W>64$, and $\left.I D=\log _{2}(2 D / W)>7\right)$. Under the deterministic iterative-corrections model, this would allow no freedom for $D / W$ to affect movement times logarithmically within the range of target distances and widths (i.e., $1 \leq$ $D / W \leq 32 ; 1 \leq I D \leq 6$ ) typically included in experiments on Fitts' law.
} 


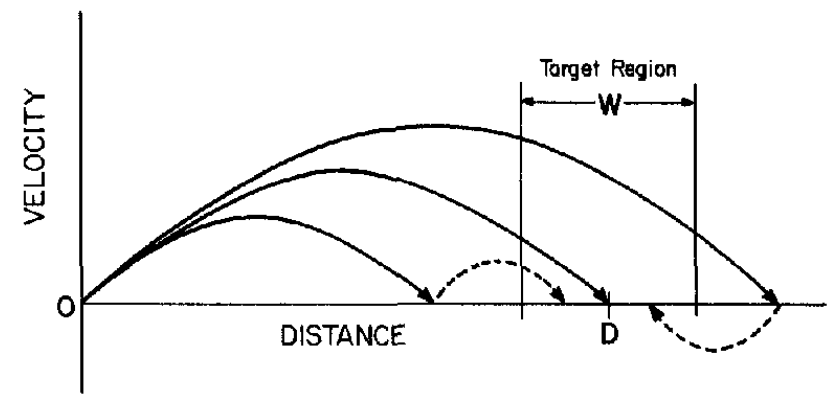

Figure 3. Outline of assumptions for the stochastic optimized-submovement model. (The horizontal axis represents movement distance and the vertical axis represents movement velocity. The solid and dashed curves correspond respectively to hypothetical primary and secondary submovements for three trials on which there are movements between an initial home position [distance $=0$ ] and a target region [bounded by vertical lines] whose center is $D$ units from the home position and whose width is $W$ units.)

ment model, noise is assumed to exist in the neuromotor system whenever a subject produces rapid aimed movements. Evidence of such noise comes from the studies of temporally constrained movements reviewed earlier (Meyer et al., 1982; Schmidt et al., 1978, 1979; Wright, 1983a, 1983b; Wright \& Meyer, 1983; Zelaznik et al., 1981, in press). Given a constant target distance and goal duration for each of several movements, subjects do not (and apparently cannot) produce exactly the same movement repeatedly across a series of trials. Instead, the movement distances and movement times deviate randomly about their intended objectives. Randomness likewise appears in other aspects of the movement trajectories (Meyer et al., 1982; Wright, 1983a, 1983b) and in bursts of electromyographic (EMG) activity associated with them (Wallace, 1981).

Fitts (1954) previously entertained the possibility that rapid aimed movements are affected by stochastic noise in neuromotor channels. As his original report stated, the "force, direction, and amplitude [of movements] are continuous variables . . . limited only by the amount of statistical variability, or noise, that is characteristic of repeated efforts to produce the same response" (Fitts, 1954, p. 381). However, following the prevalent theoretical approach of his era, he chose to interpret the effects of this noise in terms of mathematical information theory (Miller, 1953; Shannon \& Weaver, 1949), and he did not develop a detailed quantitative account of the mechanisms responsible for random movement variability. The elusive nature of neuromotor noise and the eventual loss of interest in information theory as a framework for modeling human behavior led later investigators to dismiss Fitts' (1954) ideas. Crossman and Goodeve (1963/1983, p. 283) argued that there is an "empirical difficulty in establishing the existence of the postulated 'noise.' . . . Thus, the supposed 'noise' is apparently not present in the effector systems." Their version of the deterministic iterative-corrections model, and Keele's (1968) subsequent elaboration of it, included no formal assumptions or explicit predictions about submovement-endpoint variability and the effects of neuromotor noise on it. In contrast, we will show that such noise may actually play a central role in determining how rapid spatially constrained movements are produced.

The source of the neuromotor noise postulated here is not yet known, and controversies have arisen over its detailed microscopic properties (Carlton \& Newell, 1988; Meyer et al., 1982; Schmidt, Sherwood, Zelaznik, \& Leikind, 1985; Schmidt et al., 1979). Nevertheless, certain plausible scenarios regarding random variability in the motor system do exist. According to one possible view, elementary high-velocity movements are mediated by force pulses with associated amplitude $(f)$ and time $(t)$ parameters that govern the magnitudes and durations of the pulses. When these parameters are assigned mean goal values as part of producing a desired movement, they may fluctuate randomly about their assigned values, yielding stochastic motor performance. More specifically, some theorists have proposed that, analogous to Weber's law in sensory psychophysics (Green \& Swets, 1966; Woodworth, 1938), such fluctuations entail proportional relations of the form $\sigma_{\mathrm{f}}=K_{\mathrm{f}} F$ and $\sigma_{\mathrm{t}}=K_{\mathrm{t}} T$, where $F$ and $T$ are mean values selected for the amplitude and time parameters, respectively, $\sigma_{\mathrm{f}}$ and $\sigma_{\mathrm{t}}$ are the corresponding standard deviations of these parameters, and $K_{\mathrm{f}}$ and $K_{\mathrm{t}}$ are positive constants (Meyer et al., 1982; Schmidt et al., 1978, 1979). Although some results leave this proposal open to question (Schmidt et al., 1985; Zelaznik \& Schmidt, 1983; Zelaznik, Schmidt, \& Gielen, 1986), other data support it substantially (Schmidt et al., 1978, 1979; Wright, 1983a, 1983b).

For current purposes, the stochastic optimized-submovement model does not require a complete description of the underlying mechanisms that cause neuromotor noise. We simply claim that the noise exists and that it leads to systematic variability in the endpoints of component submovements executed during the production of spatially constrained movements. Following this claim, some additional assumptions are outlined below.

Number of submovements. We assume that a rapid spatially constrained movement toward a target region includes either one or two component submovements, regardless of the target distance and width. The primary (initial) submovement is assumed to be programmed to end at the center of the target, as illustrated by one of the solid curves that start from the home position in Figure 3. If the primary submovement ends within the target region, then no secondary submovement follows it. However, the endpoint of a primary submovement may miss the target because of perturbations caused by neuromotor noise, as two of the other solid curves in Figure 3 illustrate. If a miss occurs, then a secondary submovement, prepared through visual or other (e.g., kinesthetic) feedback, is assumed to correct the error, as the dashed curves in Figure 3 illustrate.

Our motivation for proposing a model with only two submovements is partly didactic. We want to demonstrate that many aspects of the speed-accuracy trade-off associated with spatially constrained movements can be explained by hypothesizing a relatively small number of submovements. This demonstration may provide useful insights into the source of the logarithmic trade-off function (Fitts' law) and create an instructive contrast with alternative views, such as those embodied in the deterministic iterative-corrections model.

Empirical justification also exists for assuming only a primary and secondary submovement. The two-submovement assumption is supported by some results of Langolf et al. (1976) and Carlton (1979). They found a large preponderance of trials with just one or two submovements in subjects' overall movements toward a target region. The absence of additional higher 
order (c.g., tertiary) submovements seems reasonable given that a primary submovement may typically travel over $90 \%$ of the distance from the home position to the center of the target (Carlton, 1979; Vince, 1948). With this amount of travel and with the usual distance-width combinations selected for spatially constrained movement tasks, a combination of two submovements would almost always suffice to reach the target region successfully (Footnote 7). Also, when three or more submovements do occur (e.g., Crossman \& Goodeve, 1963/1983; Jaga* cinski et al., 1980), the present theoretical approach may still be appropriate in that our model can assimilate within its secondary submovements the contributions from all higher order submovements. ${ }^{8}$

Normal distribution of submovement endpoints. Because of neuromotor noise, the endpoints of the primary and secondary submovements are assumed to have normal (Gaussian) distributions around the center of the target region. This assumption is consistent with data obtained by Fitts (1954) for stylus tapping. In at least some of his studies, undershoots and overshoots of the target region were about equally frequent, corresponding to a centrally located dispersion of submovement endpoints (e.g., Fitts, 1954, Experiment 1, 1-oz stylus condition; Fitts \& Peterson, 1964). Following these results, other investigators have used an assumption of normality to infer the standard deviations of movement endpoints from observed proportions of movements that finished outside specified target regions (Crossman \& Goodeve, 1963/1983; Wallace \& Newell, 1983; Welford, 1968). ${ }^{9}$

Standard deviation of submovement endpoints. The distribution of primary-submovement endpoints is assumed to have a standard deviation, $S_{1}$, that increases proportionally with the average velocity, $V_{1}$, of the primary submovements, as expressed in the following equation:

$$
S_{1}=K V_{1}=K \frac{D_{1}}{T_{1}},
$$

where $D_{1}$ is the mean distance traveled by the primary submovements, $T_{1}$ is their mean duration, and $K$ is a positive constant. This assumption stems directly from the conceptual framework developed previously to characterize temporally constrained movements in terms of impulse-variability mechanisms (Meyer et al., 1982; Schmidt et al., 1978, 1979). Equation 2 would hold, for example, if there is a strong positive correlation between the average velocity of the primary submovements and the amplitude of the neuromotor noise associated with producing them.

Neuromotor noise is also assumed to affect the standard deviation of secondary-submovement endpoints. In particular, suppose that a primary submovement misses the target region on a trial and ends at a point $\Delta$ units from the center of the target $(|\Delta| \geq W / 2)$. Then according to the stochastic optimized-submovement model, there will be a subsequent secondary submovement programmed to travel a mean distance $\Delta$ in a mean time $T_{2 \Delta}$, and the submovement's actual endpoint in space will come from a normal distribution whose standard deviation, $S_{2}$, satisfies Equation 3:

$$
S_{2}=K \frac{\Delta}{T_{2 \Delta}}
$$

where $K$ is the same constant as in Equation 2. Because errors (target misses) are discouraged in spatially constrained movement tasks, $S_{2}$ must be sufficiently small that a large proportion (e.g., 95\%) of the secondary submovements end inside the target region. The desired $S_{2}$ is achieved by adjusting the mean duration $\left(T_{2 \Delta}\right)$ of the secondary submovements to have a value that depends on $\Delta$, on the target width, and on the required accuracy level (see Minimization of movement times).

Equations 2 and 3 are consistent with several studies that have revealed linear speed-accuracy trade-offs in temporally constrained movement tasks. For example, Schmidt et al. (1979) had subjects make discrete stylus-tapping movements from an initial home position toward a target point. Each movement was supposed to take a specified amount of time (either 140,170 , or $200 \mathrm{~ms}$ ) and to come as close as possible to the target point, given this temporal constraint. The standard deviations of movement endpoints around the target increased linearly with the average velocity of the movements. This finding has been replicated for stylus tapping by Zelaznik et al. (1981, in press) and extended to wrist rotations by Wright and Meyer (1983; also see Wright, 1983a, 1983b). ${ }^{10}$

Minimization of movement times. Under the stochastic optimized-submovement model, the primary and secondary submovements toward a target region are assumed to have average velocities programmed to minimize the average total movement time $(T)$, conditional on submovement-endpoint variability caused by neuromotor noise. Time minimization is achieved through an ideal compromise between the mean duration of the primary submovements $\left(T_{1}\right)$ and the mean duration

\footnotetext{
${ }^{8}$ The occurrence of higher order corrective submovements may depend on the extent to which a spatially constrained movement task involves extremely difficult targets (Carlton, 1980) and requires virtually errorless performance (Jagacinski, Repperger, Moran, Ward, \& Glass, 1980). In studies of disc and pin transfer, Fitts (1954) and Annett, Golby, and Kay (1958) had subjects continue moving until they were always properly aligned with a target region. Similarly, Jagacinski et al. (1980) had subjects perform wrist movements that required a cursor to be superimposed continuously on a displayed target region for at least $360 \mathrm{~ms}$. Such stringent accuracy requirements could induce additional finer and finer corrections until sufficient spatial accuracy is achieved.

${ }^{9}$ However, it should be stressed that many of the predictions made by the stochastic optimized-submovement model do not depend heavily on the present normality assumption. For example, average total movement time $(T)$ should exhibit the same form of speed-accuracy tradeoff (viz. a quasi square-root function of $D / W$ ) regardless of whether the endpoint distributions are normal or uniform. Combined with the model's other assumptions, any well-behaved symmetric unimodal endpoint distribution would lead to essentially the same predictions as a normal distribution does.

${ }^{10} \mathrm{~A}$ few studies of temporally constrained movements have suggested relations other than a linear speed-accuracy trade-off between the standard deviation of movement endpoints and the average movement velocity (e.g., Hancock \& Newell, 1985; Schmidt, Sherwood, Zelaznik, \& Leikind, 1985). Under some circumstances, spatial endpoint variability may appear to increase with the mean movement distance but be independent of movement time. Also, the constant $K$ in Equations 2 and 3 may differ for primary and secondary submovements, given that they are intended to achieve markedly different degrees of spatial precision. Such subtleties would require modifications of these equations and could lead to a somewhat different account of spatially constrained movements than outlined here.
} 
of the secondary corrective submovements $\left(T_{2}\right)$, as specified more fully later (see Quantitative Predictions).

Such a compromise is necessary for several reasons. Making primary submovements that have very high velocities would increase the frequency and magnitude with which the target region is missed and secondary submovements are required, because the standard deviation $\left(S_{1}\right)$ of the primary-submovement endpoints increases as the primary-submovement velocities increase (Equation 2). The increased secondary-submovement frequency and magnitude would yield a net increase of the average total movement time, even though the associated primary submovements have short durations. On the other hand, making primary submovements that have very low velocities would decrease the frequency and magnitude of the secondary submovements, because the primary-submovement endpoints would then tend to be closer to the target, but the net result would again be to increase the average total movement time as a result of the long primary-submovement durations. Thus, to minimize the average total movement time (i.e., $T=T_{1}+T_{2}$ ), the primary submovements should not be too fast or too slow, and correspondingly, the secondary submovements should not be too frequent or too infrequent. The values of $T_{1}$ and $T_{2}$ that yield the minimum $T$ depend on the ratio of the target distance to the target width. In general, large $D / W$ ratios require the durations of both the primary and secondary submovements to be relatively long even with submovement optimization (see Appendix).

The assumed minimization of average total movement times satisfies the task demands usually imposed on spatially constrained movements. Under circumstances like those of Fitts' (1954) stylus-tapping task, subjects are instructed to move as fast as possible without terminating their movements outside the target region on more than a small proportion of trials. Experimenters may encourage good performance through knowledge of results and appropriate monetary rewards (e.g., Fitts \& Radford, 1966). The stochastic optimized-submovement model provides an explicit statement about how these instructions should be followed.

Issues regarding the optimality of motor performance have also arisen in other related contexts. The production of aimed movements requires selecting parameters for movement trajectories along a number of related physical dimensions, including ones associated with space, time, force, and so on. An ideal actor may seek to minimize various quantities involving these parameters, such as the total distance traveled to reach some spatial position, the energy expended during movement, and the stress exerted on a moving body part. Theorists who study natural (e.g., human) and artificial (e.g., robot) movement systems have analyzed how this kind of minimization can be accomplished when movements are produced through noise-free deterministic mechanisms (e.g., Hogan, 1984; Hollerbach, 1982; Nelson, 1983). There has been some work in mathematical control theory on optimal behavior for manual tracking by nondeterministic (i.e., stochastic) mechanisms where noise plays a role (e.g., Kleinman, Baron, \& Levison, 1970). However, these efforts have not focused specifically on the speed-accuracy trade-offs exhibited by rapid spatially constrained movements nor have they treated such movements in terms of entirely realistic assumptions about the effects of neuromotor noise. This latter topic is the province of the stochastic optimized-submovement model.

Preparatory processing of information for movement production. Of course, movement production under the stochasticoptimized submovement model requires certain information to be processed ahead of time. For primary submovements, we assume that this information includes the values of the target distance and width, which are taken into account by the preparation process before initiating a primary submovement. For secondary submovements, further information is presumably taken into account as the primary submovement progresses from start to finish. A secondary submovement may be prepared on the basis of the target's location and visual or kinesthetic feedback regarding dynamic characteristics (e.g., velocity and acceleration) of the primary submovement. The process that prepares secondary submovements may also use "feedforward" (efference copy) from the primary submovements (von Holst, 1954). ${ }^{.1}$

We assume that feedback and feedforward are processed "on the fly" during movement production, so that essentially no delay ensues between the end of a primary submovement and the beginning of a subsequent secondary submovement. As indicated already, the stochastic optimized-submovement model simply sums the durations of the primary and secondary submovements in order to represent the total movement time on a trial. The model does not allow for "dead time" (i.e., intermediate pauses during which movement velocity is zero over an extended time interval) after a primary submovement has begun. This constraint simplifies computation of the mean submovement durations that minimize the average total movement time (see Appendix), and it is consistent with most of the movement trajectories found in the experiments reported here.

\section{Quantitative Predictions}

Using the stochastic optimized-submovement model, we have derived a number of quantitative predictions about rapid spatially constrained movements. The predictions concern the average total movement time, mean durations of primary and secondary submovements, spatial variability (standard deviation) of primary-submovement endpoints, relative frequency of secondary submovements, and relative frequency of errors (i.e., target misses). For each of these dependent variables, a prediction can be made as a function of target distance and width.

To facilitate our presentation, we will outline the model's predictions in two complementary forms. First, in the main text, several of the predictions are introduced as simplified approximations involving relatively nontechnical notation. Second, in the accompanying footnotes and Appendix, the predictions are stated more precisely with technically exact notation. Table 1 summarizes both the approximate and exact forms of the pre-

\footnotetext{
"This would involve comparing a set of intended motor commands against copies of the actual motor commands sent to the effectors for producing a desired primary submovement. If the intended and actual commands differ from each other, then their difference could provide a way of deciding what correction(s) to make during subsequent secondary submovements. The efference-copy correction mechanism might be entirely central in nature, not requiring any peripheral feedback (von Holst, 1954).
} 
dictions. Also included in Table 1 are illustrative numerical values derived from these predictions for representative target distances and widths. Complete derivations of the model's predictions appear in the Appendix.

Average total movement time. The stochastic optimized-submovement model predicts that the average total movement time is closely approximated by

$$
T=A+B \sqrt{D / W}
$$

where $A$ and $B$ are nonnegative constants. ${ }^{12}$ This equation, which involves a square-root function of $D / W$, has some interesting properties. According to it, $T$ should vary in a negatively accelerated fashion with $D / W$, even though the standard deviations of the primary-submovement and secondary-submovement endpoints are governed by the same linear trade-off function (i.e., Equations 2 and 3) as temporally constrained movements are. Also, because $\sqrt{D / W}$ increases monotonically at a decreasing rate as $D / W$ increases, Equation 4 exhibits a shape similar to $\log _{2}(2 D / W)$, mimicking Fitts' law. The degree of similarity is illustrated in Table 1, where we have fit Equation 1 (Fitts' law) to illustrative numerical values derived from the stochastic optimized-submovement model. Here it can be seen that the square-root and logarithmic trade-offs come fairly close to each other $(r=.97)$ except for small and large values of $D / W$, where the greater curvature of the logarithmic trade-off yields moderately large deviations. ${ }^{13}$

The square-root trade-off function predicted by the stochastic optimized-submovement model is also consistent with previous results in the literature. Several investigators have reported data for which a power function with an exponent of about onehalf describes the speed-accuracy trade-off in spatially constrained movements better than a logarithmic function does (Kvalseth, 1980). Observed deviations of empirical data from Fitts' law have tended to reveal a slight upward curvature when average total movement times $(T)$ are plotted versus $\log _{2}(2 D /$ $W)$, as expected from Equation $4 .{ }^{14}$

There are some circumstances in which Equation 4 might not apply, and $T$ might instead be a linear function of $D / W$ for spatially constrained movements. This would happen if subjects restricted their movement attempts to lone primary submovements, omitting secondary and higher order corrective submovements. Using a one-submovement strategy, they could perform a spatially constrained movement task by programming the primary submovements to have a mean duration $T_{1}$ such that $T_{1}=T=2 K z_{\mathrm{c} 2}(D / W)$, where $c_{2}$ is the desired frequency of correct movement trials (i.e., target hits), and $z_{\mathrm{e} 2}$ is a unit-normal deviate such that a proportion $c_{2}$ of the area under a standardized normal probability-density function falls inside the interval $\left(-z_{\mathrm{c} 2}, z_{\mathrm{c} 2}\right)$. Then performance would mimic the results typically observed for temporally constrained movements (e.g., Schmidt et al., 1979; Wright \& Meyer, 1983). Under a broad range of conditions, however, the one-submovement strategy would not be as optimal as a two-submovement strategy with respect to movement-time minimization. When $D / W>4 /$ $z_{\mathrm{c} 2} \sqrt{2 \pi}$, two (i.e., a primary and optional secondary) submovements of the sort incorporated in the stochastic optimized-submovement model can yield an average total movement time $(T)$ that is shorter than would be possible with one primary submovement whose accuracy achieves the same desired level as the two-submovement strategy does (see Appendix). ${ }^{15}$
Mean primary-submovement duration. For primary submovements, the stochastic optimized-submovement model predicts that the mean duration is approximated by

$$
T_{1}=A_{1}+B_{1} \sqrt{D / W}
$$

where $A_{1}$ and $B_{1}$ are positive constants $\left(A_{1}<A\right.$, and $\left.B_{1}<B\right)$. The primary-submovement durations should therefore mimic Fitts' law in the same fashion as do the average total movement times. ${ }^{16}$

Equation 5 agrees well with the findings of some previous investigators. As mentioned earlier, Langolf et al. (1976, Experiment 2) found that primary submovements in stylus tapping had durations whose magnitudes depended on both the target distance and width; farther and narrower targets induced primary submovements whose durations were longer. A similar result occurred in the experiment by Jagacinski et al. (1976). This is expected under the stochastic optimized-submovement

\footnotetext{
${ }^{12}$ More precisely, the average total movement time is given by $T=$ $2 K(2 \theta \sqrt{D / W}-\sqrt{W / D}) / \theta \sqrt{\theta-(W / D)}$. Here $K$ is the multiplicative constant from Equations 2 and 3 for the standard deviations of primarysubmovement and secondary-submovement endpoints. $\theta$ is a parameter whose value converges to $\sqrt{2 \pi} / z_{\mathrm{c} 2}$ as $D / W$ grows large. The constant $z_{\mathrm{c} 2}$ is a unit-normal deviate ( $z$-score) such that a proportion $c_{2}$ of the area under a standardized normal probability-density function with zero mean and unit variance falls inside the interval $\left(-z_{c 2}, z_{c 2}\right)$. The proportion $c_{2}$ corresponds to the probability that a secondary submovement ends correctly inside the target region, given that a secondary submovement is produced. As we show in the Appendix, Equation 4 becomes an especially close approximation as $D / W$ grows large (i.e., as more spatial precision is required in a movement), because the quantity $W / D$ approaches zero, leading to $A=0$ and $B=4 K\left(z_{c 2} / \sqrt{2 \pi}\right)^{1 / 2}$ in Equation 4 . The closeness of the approximation can be judged from Table 1 .

${ }^{13}$ Mathematically, $\log _{2}(D / W)$ is equivalent to the limiting case of a power function of $D / W$ whose positive exponent approaches zero. For power functions of the form $(D / W)^{x}$, where $0<x<1$, the degree of curvature is greater than that of a linear function (i.e., one where $x=$ 1) but less than that of the corresponding logarithmic function.

${ }^{14}$ A square-root function comes closer than a logarithmic function to some of Fitts' (1954, Table 1, p. 385) own data. In his 1-oz stylus condition, $r^{2}=.974$ for Equation 4, whereas $r^{2}=.966$ for Equation 1 . In his 1-lb stylus condition, $r^{2}=.972$ for Equation 4, whereas $r^{2}=.960$ for Equation 1. This is what would be expected under the stochastic optimized-submovement model.

${ }^{15}$ These optimality considerations explain some results obtained by investigators who have reported violations of Fitts' law for very near or wide target regions (e.g., Klapp, 1975). If the preceding inequality does not hold (i.e., $D / W<4 / z_{\mathrm{c} 2} \sqrt{2 \pi}$ ), then the one-submovement strategy may be optimal, and subjects may adopt it in preference to a strategy involving two or more submovements, thereby preempting a quasi-logarithmic trade-off function as embodied in Equation 4 . This possibility would arise, for example, if $D / W$ were less than .8 and subjects wanted to make correct movements with a relative frequency of .95 , because then $D / W<4 / z_{\mathrm{c} 2} \sqrt{2 \pi}=.81$.

${ }^{16}$ More precisely, the mean primary-submovement duration is given by $T_{1}=2 K \sqrt{D / W} / \sqrt{\theta-(W / D)}$. Here $K$ is the same constant as in Equations 2 and 3 for the standard deviations of primary-submovement and secondary-submovement endpoints, and $\theta$ is a positive parameter discussed elsewhere (Appendix and Footnote 12). As $D / W$ grows large, the quantity $W / D$ approaches zero, and the denominator of $T_{1}$ approaches $\sqrt{\theta}$, making $T_{1}$ be an essentially pure square-root function of $D / W$, just like the average total movement time is (cf. Equation 4 ). The closeness of the approximation can be judged in Table 1 .
} 
Table 1

Summary of Exact and Approximate Quantitative Predictions Made by the Stochastic Optimized-Submovement Model

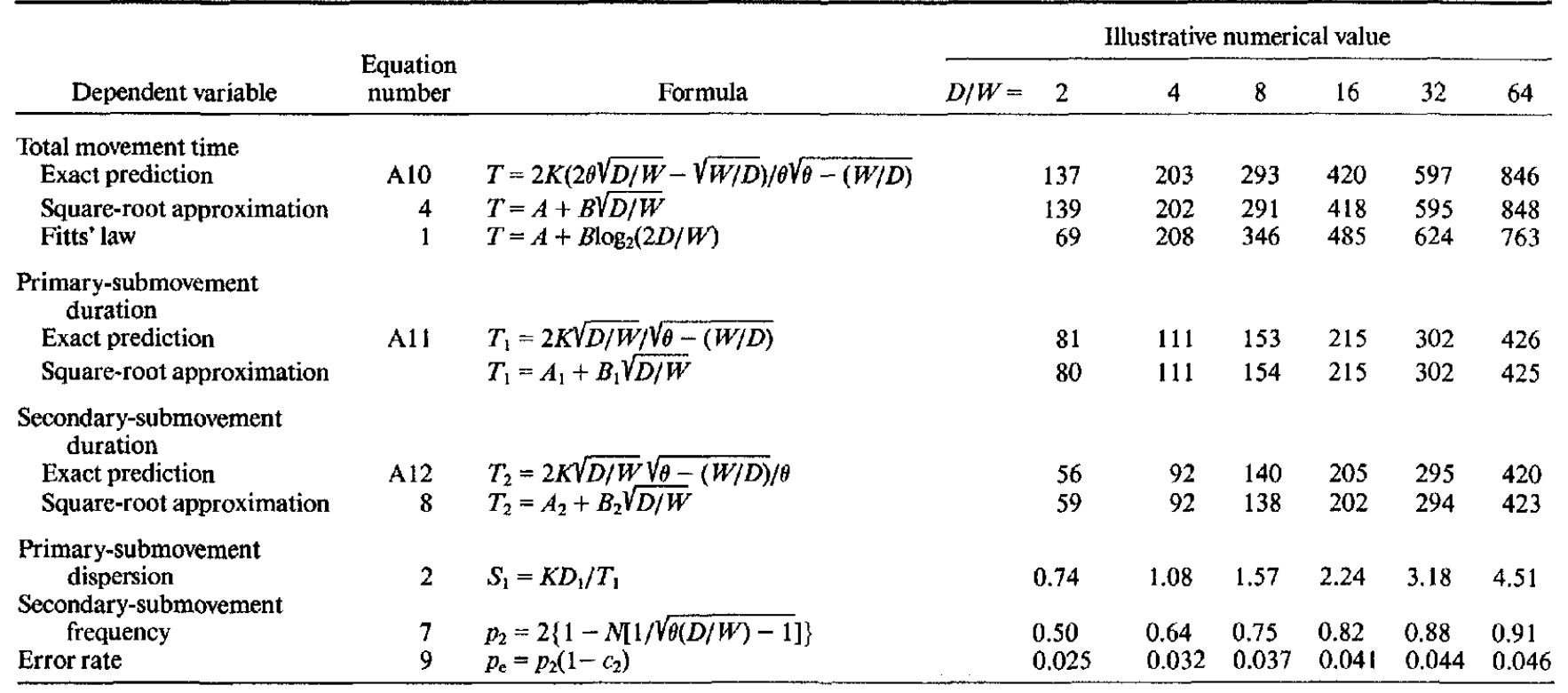

Note. In the above formulas, $D$ is target distance, $W$ is target width, $K$ is the slope of the linear speed-accuracy trade-off (Equations 2 and 3 ), and $c_{2}$ is the probability that a secondary submovement ends inside the target region. The values derived from the exact predictions of the model assume $K=30 \mathrm{~ms}, c_{2}=.95$, and $W=1$. The parameter $\theta$, whose value depends on $D$ and $W$, may be calculated in terms of Equations $A 8$ and $A 9$ (Appendix). In the square-root approximations to the model, values were obtained by selecting the intercept and slope parameters $A, B, A_{1}, B, A$, and $B_{2}$ to maximize the goodness of fit with the exact predictions. This yielded $A=-13, B=108$, and $r=.99$ for Equation 4 versus $\mathrm{Al} 10 ; A_{1}=6$, $B_{1}=52$, and $r=.99$ for Equation 5 versus $A 11 ; A_{2}=-19, B_{2}=55$, and $r=.99$ for Equation 8 versus A12. The parameters $A$ and $B$ in Equation 1 (Fitts' law) were selected to maximize the goodness of fit with the values derived from Equation A10. This yielded $A=-209, B=138$, and $r=$ 97.

model. In essence, wider targets provide more room for the endpoints of successful primary submovements, thereby allowing the primary submovements to have higher average velocities without excessively increasing the risk of missing the target region because of amplified neuromotor noise. So when given the goal of minimizing the average total movement time, subjects should decrease the durations of their primary submovements whenever the width of a target at a fixed distance is increased. Nearer targets would likewise allow the primary submovements to have shorter durations without increasing the dispersions of their endpoints too greatly (Equation 2). Evidence for Equation 5 can be viewed as supporting a combination of the model's assumptions about the effects of neuromotor noise on primarysubmovement endpoints and about the minimization of average total movement times.

Standard deviation of primary-submovement endpoints. Complementing its predictions about movement times, the stochastic optimized-submovement model makes a prediction about the standard deviation of primary-submovement endpoints in space. This prediction follows directly from the model's assumptions regarding the effects of neuromotor noise. The endpoints of the primary submovements should have a standard deviation $\left(S_{1}\right)$ that increases linearly with the average primary-submovement velocity $\left(D_{1} / T_{1}\right)$, satisfying Equation 2 . As described already, such linearity has been observed by several investigators for temporally constrained movements (Schmidt et al., 1978, 1979; Wright, 1983a, 1983b; Wright \& Meyer, 1983; Zelaznik et al., 1981, in press). However, no analo- gous observation has been made yet regarding spatially constrained movements.

Relative frequency of secondary submovements. On the basis of variability in the primary-submovement endpoints, an exact prediction can also be made about the relative frequency of secondary submovements as a function of target distance and width. Let $c_{1}$ denote the probability that a primary submovement ends inside the target region, and let $p_{2}$ denote the relative frequency of secondary submovements. Also, let $N[1 /$ $\sqrt{\theta(D / W)-1}$ denote the probability that a random variable with a standard normal distribution (i.e., one having zero mean and unit variance) is less than $1 / \sqrt{\theta(D / W)}-1$, where $\theta$ is a parameter discussed elsewhere (see Appendix, Footnote 12 and Footnote 16). Then according to the stochastic optimized-submovement model,

$$
\begin{gathered}
c_{1}=2 N[1 / \sqrt{\theta(D / W)-1}]-1, \\
p_{2}=1-c_{1}=2\{1-N[1 / \sqrt{\theta(D / W)-1}]\} .
\end{gathered}
$$

From Equations 6 and 7, we see that the predicted relative frequency of secondary submovements increases continuously as $D / W$ increases. This follows because $p_{2}$ increases as $c_{1}$ decreases (Equation 7), and $c_{1}$ decreases as $D / W$ increases (Equation 6). In effect, the use of more secondary submovements with increasing $D / W$ allows the average total movement time to remain relatively short despite the requirement of greater spatial movement precision. To achieve such precision through primary submovements alone would require excessively long pri- 
mary-submovement durations, given the effects of neuromotor noise on the variability of primary-submovement endpoints (Equation 2).

Mean secondary-submovement duration. Because the average total movement time $(T)$ is expressed as a sum of the mean primary-submovement duration $\left(T_{1}\right)$ and the mean secondarysubmovement duration $\left(T_{2}\right)$, we may subtract Equation 5 from Equation 4 to obtain a complementary expression for the mean secondary-submovement duration; that is,

$$
T_{2}=T-T_{1}=A_{2}+B_{2} \sqrt{D / W},
$$

where $A_{2}$ and $B_{2}$ are positive constants such that $A_{2}=A-A_{1}$ and $B_{2}=B-B_{1}$, respectively. ${ }^{17} T_{2}$, like $T_{1}$, should therefore increase in a negatively accelerated fashion with $D / W$. This prediction is supported by the results of some investigators who have found that target distance and width influence the duration of each submovement in a submovement series (e.g., Jagacinski et al., 1980). ${ }^{18}$

Error rates. Combining its assumptions about submovement durations and standard deviations of submovement endpoints, the stochastic optimized-submovement model also makes predictions regarding error rates (i.e., relative frequencies of trials on which a subject finishes without having hit the target region). Because there are supposed to be at most two submovements per trial, an error will occur if, and only if, the primary and secondary submovements both end outside the target region. We denote the probabilities associated with these events as 1 $c_{1}$ and $1-c_{2}$, respectively, where $c_{1}$ and $c_{2}$ are the corresponding probabilities that the primary and secondary submovements end inside the target region. Equation 7 characterizes $1-c_{1}$ as a function of $D / W$, and $1-c_{2}$ is assumed to be constant. Consequently, the mean error rate, $p_{\mathrm{e}}$, should satisfy Equation 9:

$$
\begin{aligned}
& p_{\mathrm{e}}=\left(1-c_{1}\right)\left(1-c_{2}\right) \\
&=2\left(1-c_{2}\right)\{1-N[1 / \sqrt{\theta(D / W)-1}]\}
\end{aligned}
$$

where $N[1 / \sqrt{\theta(D / W)-1}]$ has been defined previously (see $R e l$ ative frequency of secondary submovements; Equations 6 and 7 ).

Equation 9 implies that the error rate $\left(p_{\mathrm{e}}\right)$ increases monotonically with $D / W$. The reason for this is simple. Under the stochastic optimized-submovement model, there are only two chances for hitting the target region on each trial. One of these involves the primary submovements, and the other involves the secondary submovements. As $D / W$ grows large, the probability that a secondary submovement misses the target (i.e., $1-c_{2}$ ) is assumed to stay the same, but the probability that a primary submovement misses the target (i.e., $1-c_{1}$ ) increases (Equation 7). So the overall chance of a correct outcome decreases, and the error rate increases. The rate of increase in $p_{c}$ with $D / W$ will be less than the rate of increase in misses by the primary submovements because of the contribution from subsequent correct secondary submovements.

No detailed tests of predictions about error rates have appeared in past literature on rapid spatially constrained movements. Fitts (1954) did, however, find that target misses increased with $D / W$ for stylus tapping. A similar finding has also been reported by Wallace and Newell (1983) and by some other
Table 2

Comparisons of Predictions Made by the Stochastic Optimized-Submovement Model and the Deterministic Iterative-Corrections Model

\begin{tabular}{cll}
\hline & \multicolumn{2}{c}{ Predicted effects of increasing $D / W$} \\
\cline { 2 - 3 } $\begin{array}{c}\text { Dependent } \\
\text { variable }\end{array}$ & $\begin{array}{l}\text { Stochastic optimized- } \\
\text { submovement model }\end{array}$ & $\begin{array}{c}\text { Deterministic iterative- } \\
\text { corrections model }\end{array}$ \\
\hline$T$ & Square-root increase & Logarithmic increase \\
$T_{1}$ & Square-root increase & None \\
$T_{2}$ & Square-root increase & None \\
$S_{1}$ & Increase & None \\
$p_{2}$ & Continuous increase & All-or-none increase \\
$p_{\mathrm{e}}$ & Increase & None \\
\hline
\end{tabular}

Note. $T=$ average total movement time; $T_{1}=$ mean primary-submovement duration; $T_{2}=$ mean secondary-submovement duration; $S_{1}=$ standard deviation of primary-submovement endpoints; $p_{2}=$ relative frequency of secondary submovements; $p_{\mathrm{e}}=$ error rate; $D=$ distance; and $W=$ width.

investigators (e.g., Fitts \& Peterson, 1964). These results are qualitatively consistent with the stochastic optimized-submovement model. Still, it remains to be determined whether the effects of target distance and width on mean error rates support the model quantitatively.

\section{Summary of Predictions}

In summary, Table 2 compares various predictions made by the stochastic optimized-submovement model and the deterministic iterative-corrections model. Six key dependent variables are covered, including the average total movement time $(T)$, mean primary-submovement duration $\left(T_{1}\right)$, standard deviation of primary-submovement endpoints $\left(S_{1}\right)$, relative frequency of secondary submovements $\left(p_{2}\right)$, mean secondary-submovement duration $\left(T_{2}\right)$, and error rate $\left(p_{\mathrm{e}}\right)$. For each variable, the models differ with respect to the expected effects of increasing $D / W$. In most cases, these differences are considerable, and

\footnotetext{
${ }^{17}$ Bear in mind that by definition, $T_{2}$ includes a contribution from each movement trial, regardless of whether a secondary submovement actually occurs there. For trials on which a primary submovement but no secondary submovement takes place, the observed secondary-submovement duration is set equal to zero as part of calculating $T_{2}$. To calculate the mean secondary-submovement duration for only those trials on which secondary submovements do take place, one would divide $T_{2}$ by $p_{2}$, the relative frequency of secondary submovements.

${ }^{18}$ More precisely, it can be shown that under the stochastic optimized-submovement model, $T_{2}=2 K \sqrt{D / W} \sqrt{\theta-(W / D)} / \theta$, where $K$ and $\theta$ are parameters discussed elsewhere (see Equations 2 and 3, Footnote 12 , Footnote 16 , and Appendix). For moderate $D / W$, the latter expression is less than $T_{1}$, the mean primary-submovement duration (cf. Footnote 16). As $D / W$ grows large, however, both $T_{1}$ and $T_{2}$ would approach the value $2 K \sqrt{D / W} / \sqrt{\theta}$, so nearly equal amounts of time would be devoted on the average to the primary and secondary submovements for difficult targets. This convergence implies that as $D / W$ grows large, the stochastic optimized-submovement model tends in some respects to mimic the deterministic iterative-corrections model (Crossman \& Goodeve, 1963/1983; Keele, 1968), which assumes that all submovements take exactly the same time increment.
} 
the deterministic iterative-corrections model fails to handle previously reported data, whereas the stochastic optimized-submovement model is at least qualitatively consistent with them.

\section{Overview of Experiments}

To obtain further tests of the stochastic optimized-submovement model, we conducted two experiments on rapid spatially constrained movements with and without concurrent visual feedback. Each experiment involved a wrist-rotation task. This task required subjects to turn a handle quickly and accurately from an initial home position to a specified target region. Figure 4, which comes from a prior study by Wright and Meyer (1983), illustrates the type of physical set-up adopted here for presenting stimulus displays and recording individual movements.

\section{Rationale for Studying Wrist Rotations}

We chose to study wrist rotations for several reasons. (a) As mentioned earlier, wrist rotations exhibit a linear speed-accuracy trade-off in temporally constrained movements (Wright, 1983a, 1983b; Wright \& Meyer, 1983) but obey Fitts' law in spatially constrained movement tasks (Crossman \& Goodeve, 1963/1983; Jagacinski et al., 1980). This makes the wrist-rotation task an appropriate medium for testing the predictions of the stochastic optimized-submovement model. (b) Unlike stylus tapping and other multijointed movements, wrist rotations have only one degree of spatial freedom, simplifying analyses of movement kinematics. (c) Wrist rotations involve relatively high torque and low inertia, allowing the actions of muscles to be observed without a great deal of ancillary "filtering" by passive mechanical characteristics of the bones and joints (Partridge, 1967). (d) Wrist rotations may be stopped entirely by friction and antagonist muscle activity, thus precluding contamination from physical impact with the target region, which might distort records of movements in the way that stylus tapping does (cf. Schmidt et al., 1979, Figure 1).

\section{Stimulus Displays}

Information that subjects received from the stimulus displays while performing the wrist-rotation task in each experiment was controlled precisely. Representations of the home position, target region, and movement were displayed schematically on a video screen (CRT). Subjects were not allowed to view their hands directly while producing the required movements. This helped dissociate visual feedback from kinesthetic feedback, and it made kinesthetic feedback less useful for secondary corrective submovements, because kinesthesis could not be evaluated directly with respect to the spatial positions of the displayed movements and target regions. Consequently, apparent similarities between the subjects' movements under the different feedback conditions of Experiments 1 and 2 may be attributed mainly to the nature of primary submovements prepared before movement initiation (Woodworth, 1899).

\section{Movement Parsing}

Another important feature of the present experiments is an algorithm for parsing aimed movements into component submovements. We evolved the parsing algorithm by modifying

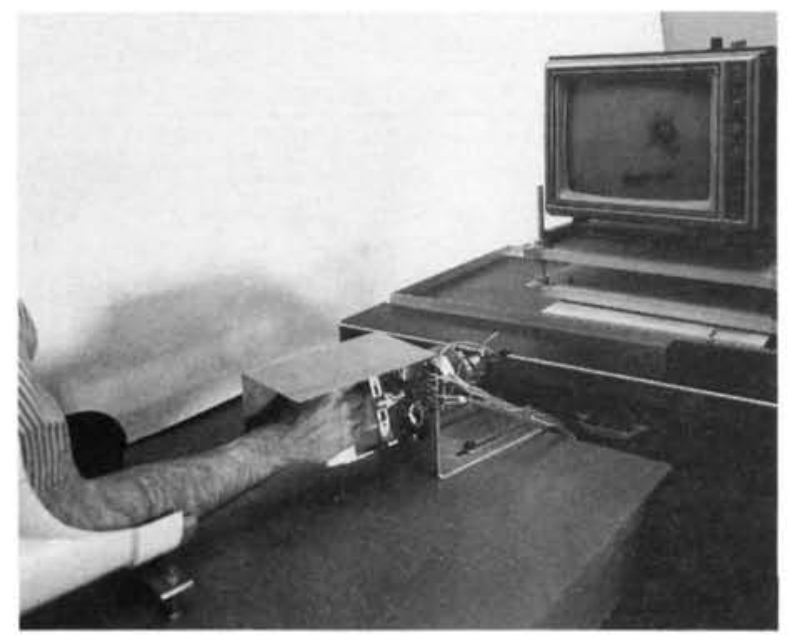

Figure 4. Physical setup for a wrist-rotation task similar to the one used in Experiments 1 and 2. (From "Conditions for a linear speed-accuracy trade-off in aimed movements" by C. E. Wright \& D. E. Meyer, Quarterly Journal of Experimental Psychology. 1983, 35A, 279-296. Reprinted with permission of author and publisher.)

and extending analytical techniques that previous investigators have applied to detect corrective submovements (e.g., Carlton, 1980; Jagacinski et al., 1980; Langolf et al., 1976; Wright, $1983 a, 1983 b$ ). Our approach entails a systematic evaluation of the velocity and acceleration records derived from each movement trajectory. In this evaluation, submovements are defined on the basis of certain criterion events, for example, crossings of well-defined velocity thresholds. These criteria are chosen to take account of known differences between the dynamics of voluntary movements (Meyer et al., 1982) and passive residual activity due to the springlike characteristics of muscles (Bizzi, Dev, Morasso, \& Polit, 1978; Fel'dman, 1966; Sakitt, 1980) and physiological tremor (Stein \& Lee, 1981). A detailed description of the movement-parsing algorithm used here appears in the method section of Experiment 1.

\section{Experiment 1}

The first experiment investigated subjects' production of spatially constrained movements made with full visual feedback. This allowed us to test the stochastic optimized-submovement model's predictions about primary and secondary submovements when the secondary submovements were reasonably accurate.

\section{Method}

Subjects. Four right-handed University of Michigan students served as paid subjects. Each subject was tested individually. The subjects came from a pool of volunteers at the Human Performance Center. They had no apparent visual or motor defects. We paid them $\$ 4$ per hour plus bonuses based on good performance.

Apparatus. Each subject sat in a sound-attenuation booth, with the right arm resting on a horizontal wooden platform at about waist height. The upper part of the arm was placed next to the body, and the forearm was parallel to the floor. Using the right hand, the subject grasped a light wooden handle that fit comfortably in the palm. The handle rotated 
Table 3

Combinations of Target Distance $(D)$ and Width $(W)$ in Degrees of Wrist Rotation Used for Experiments 1 and 2

\begin{tabular}{llrrr}
\hline$W$ & $D$ & $D / W$ & $\sqrt{D / W}$ & $\log _{2}(2 D / W)$ \\
\hline \multirow{2}{*}{1.61} & 15.81 & 9.85 & 3.14 & 4.30 \\
& 25.00 & 15.57 & 3.95 & 4.96 \\
2.54 & 10.00 & 3.94 & 1.98 & 2.98 \\
& 15.81 & 6.23 & 2.50 & 3.64 \\
& 25.00 & 9.85 & 3.14 & 4.30 \\
& 39.52 & 15.57 & 3.95 & 4.96 \\
4.01 & 10.00 & 2.49 & 1.58 & 2.32 \\
& 15.81 & 3.94 & 1.98 & 2.98 \\
& 25.00 & 6.23 & 2.50 & 3.64 \\
& 39.52 & 9.85 & 3.14 & 4.30 \\
6.34 & 15.81 & 2.49 & 1.58 & 2.32 \\
& 25.00 & 3.94 & 1.98 & 2.98 \\
\hline
\end{tabular}

freely along the axis of the forearm with low inertia and friction. A shield hid the hand and handle from the subject's view. Attached to the handle was an angular-position transducer (Brush Instruments Model 33-04) that converted the handle's position to a voltage. The voltage was digitized by an A-to-D converter at a rate of $1000 \mathrm{~Hz}$ with a resolution of $\pm .05^{\circ}$ of handle arc. Visual displays for the movements and feedback appeared on a CRT screen (DEC Model VR17, with P4 phosphor). The screen was located $43 \mathrm{~cm}$ in front of the subject. Warning tones and response tones were presented over a loudspeaker located $1 \mathrm{~m}$ behind the subject's head. A digital computer (DEC PDP-1 1/60) controlled the sequence of events and collection of data.

Design. Each subject participated in six 50-min sessions over a 2week period. The first two sessions constituted a practice period. Data from them were not included in the analyses that follow. The subsequent four test sessions provided the reported results.

There were 12 blocks of trials per session. Each block included 4 warm-up trials followed by 12 test trials. All trials on which a subject started moving too soon (i.e., before a designated response tone) or failed to complete the movement within $1 \mathrm{~s}$ after starting it were discarded and rerun at the end of the block.

The experiment involved 12 different target conditions, corresponding to various combinations of target distance and width, as shown in Table 3. Four levels of distance and four levels of width were included. The target distances ranged from $10.00^{\circ}$ to $39.52^{\circ}$ of wrist rotation relative to the initial home position of the handle. The target widths ranged from $1.61^{\circ}$ to $6.34^{\circ}$ of wrist rotation.

The distance-width combinations for the 12 targets were arranged to form a semiorthogonal factorial design (Winer, 1962). This yielded five levels of $D / W$, ranging from 2.49 to 15.57 . Over these five levels, the value of $\sqrt{D / W}$ ranged from 1.58 to 3.95 . The associated ID values ranged from 2.32 bits to 4.96 bits, typical of ones chosen by previous investigators (e.g., Crossman \& Goodeve, 1963/1983; Fitts, 1954). ${ }^{19}$

One of the 12 target conditions was assigned to each trial block. This assignment changed from block to block so that over the course of a session, a subject performed equally often with each distance-width combination. The order of the targets was counterbalanced across sessions and subjects, using a Greco-Latin square design.

Procedure. Each trial started with an initial visual display that included a fixation dot on the left of the CRT screen, a target region to the right of the fixation dot, and a movable cursor. The fixation dot indicated the home position for the required movement. The target region was defined by two vertical lines whose distance from each other represented the width of the target. The target distance corresponded to the distance from the fixation dot to the midpoint between these lines. The cursor was a small triangle whose top corner indicated the current position of the handle. Clockwise and counterclockwise rotations of the handle produced respective rightward and leftward horizontal movements of the cursor across the screen. The horizontal position of the cursor was a linear function of the angular position of the handle. Each degree of wrist rotation moved the cursor $0.29^{\circ}$ of visual angle on the display screen. Conversely, each degree of visual angle on the display screen was equivalent to $3.45^{\circ}$ of handle rotation. Thus, when the fixation dot, target region, and cursor were visible, the subject had complete information regarding the positions of both the handle and the target.

When the initial visual display for a trial appeared, the subject turned the handle to align the top corner of the cursor with the fixation dot. This required turning the right wrist counterclockwise $10^{\circ}$ from vertical. After the cursor had been aligned with the fixation dot for $800 \mathrm{~ms}$, the fixation dot changed to a plus sign. Then a 600 -ms foreperiod ensued. The foreperiod was followed by four 50 -ms tones, each one separated from the others by an interval of $260 \mathrm{~ms}$. The first three tones had a $400-\mathrm{Hz}$ frequency and served as warning signals. The fourth tone, whose frequency was $1000 \mathrm{~Hz}$, served as a response signal to initiate the required movement. At the onset of the response signal, the subject had to rotate the handle and move the cursor quickly from the home position to the target region. All of the required movements were clockwise rotations of the right wrist. The cursor remained visible throughout each trial, providing visual feedback about the progress of the movement.

We instructed the subject to reach the target region quickly and accurately once a movement had begun. If the movement did not start within $400 \mathrm{~ms}$ after the response signal, then a message ("Started Too Late") was presented, and the trial was repeated. However, subjects were not pressured to minimize their movement latency (i.e., the time between the response signal and movement onset). There was no penalty for late starts; only the duration between the start and stop of a movement (i.e., the movement time) was supposed to be minimized. Subjects were rewarded for short movement durations, provided that their movements ended inside the target region. Thus, it was advantageous for them to be both fast and accurate once a movement began.

Various types of information were presented after each trial, depending on the subject's performance. These included a display showing the fixation dot, target region, and final position of the cursor at the moment when the movement nominally ended, together with the number of points earned on the trial. The points were an inverse linear function of the movement time. For each movement that ended inside the target region, the subject earned 80 points minus 1 point per each $10 \mathrm{~ms}$ in the total movement time. For any movement that ended outside the target region, the subject earned no points. The information display lasted $1 \mathrm{~s}$ and gave the subject complete knowledge of results about the spatial accuracy and duration of the movement. Following this display, a blank interval of $1 \mathrm{~s}$ occurred before the next trial.

At the end of a trial block, the subject saw a summary display with the total number of points earned during the block and the number of trials on which a movement did not end inside the target region. This information was discussed with the subject. Subjects were continually encouraged to decrease their movement times and to stop accurately inside the target. Bonus payments depended directly on the total number of points accumulated over a session. The points were converted to money at a rate of $1 \$$ per 10 points.

${ }^{19}$ For each $I D$ value, either two or three different distances and widths were represented among the 12 target conditions. We accomplished this by (a) selecting target distances such that each one was a factor of 1.581 greater than its immediate predecessor and (b) selecting target widths such that each one was also a factor of 1.581 greater than its immediate predecessor. As a result, the effects of target distance and width may be assessed separately to determine whether they are independent and compensatory, which Fitts's law implies they should be (Sheridan, 1979; Welford, 1968). 
Movement parsing. In parsing the subjects' movements to isolate primary and secondary submovements, we first smoothed and differentiated the records of handle position as a function of time on each trial. The smoothing process involved three steps: (a) Each movement record was passed through a digital filter with a $30-\mathrm{Hz}$ low-pass cutoff, which removed any spurious electrical noise (e.g., 60-cycle hum) in the transduced signal. (b) The filtered records of position-by-time were passed twice through a $30-\mathrm{Hz}$ low-pass differentiating filter, yielding derived records of velocity and acceleration versus time, uncontaminated by noise from the differentiation process. (c) The filtered position, velocity, and acceleration records from the first two steps were each smoothed further by passing them through a final, more stringent, low-pass filter. This filter had a pass band that ranged from 0 to $7 \mathrm{~Hz}$, a skirt that ranged from $7 \mathrm{~Hz}$ to $9 \mathrm{~Hz}$, and a stop band that ranged from $9 \mathrm{~Hz}$ to infinity. It helped attenuate frequency components associated with physiological tremor (Stein \& Lee, 1981) and passive springlike oscillatory characteristics of the muscles (Bizzi et al., 1978). ${ }^{20}$

Next we applied our movement-parsing algorithm to locate the start of the primary submovement on each trial, using the velocity records derived from the preceding three substeps. The primary-submovement start was defined to be at the first moment in time after the response signal when the angular velocity of the handle exceeded $4^{\circ}$ per second and remained above that level continuously for at least $20 \mathrm{~ms}$. These criteria were selected so that the onsets of voluntary wrist rotations, but not involuntary tremor, would be detected as primary-submovement starts. The position, velocity, and acceleration of the handle at the identified starts of the primary submovements were saved for further analyses.

Having found the start of the primary submovement on a trial, we searched the corresponding velocity record to find the first relative maximum (i.e., peak in velocity) thereafter. This maximum provided an intermediate point from which we proceeded to identify the end of the primary submovement. The primary-submovement end was defined to be at the first subsequent moment such that either (a) the velocity crossed zero, changing from positive to negative, (b) the acceleration crossed zero, changing from negative to positive, or (c) a relative minimum occurred in the absolute value of the acceleration while the signed acceleration was negative. These three criteria may be interpreted as reflecting alternative types of transitions from one voluntary submovement to the next (Carlton, 1980; Jagacinski et al., 1980). ${ }^{21}$ The pasition, velocity, and acceleration of the handle at the identified ends of the primary submovements were saved for further analyses. Also, the duration of each primary submovement was defined to be the amount of time from the start to the end of the primary submovement.

Then we checked for evidence of a secondary submovement after the end of each primary submovement. This involved finding the earliest subsequent moment such that the angular velocity of the handle fell within a range of $\pm 12^{\circ}$ per second and remained there continuously for at least $160 \mathrm{~ms}$, satisfying a "stop-and-hold" criterion (Wright, 1983a, 1983b). The end of the overall movement was defined tentatively to be at the moment when the latter stop-and-hold criterion (i.e., absolute value of velocity $\leq 12^{\circ}$ per second) first became satisfied. Given the latter definition, a search was done over the interval obtained between the end of the primary submovement and the tentative end of the overall movement. If this interval had a duration of at least $60 \mathrm{~ms}$, if the movement velocity exceeded an absolute value of $4^{\circ}$ per second at some moment during the interval, and if a net distance of $1^{\circ}$ or more in angular handle position was traversed during the interval, then it was deemed to contain a secondary submovement; otherwise, it was not. These criteria helped ensure that the putative secondary submovements stemmed from voluntary activity rather than physiological tremor or passive damped oscillations at the ends of the primary submovements. When the criteria were satisfied, we defined the duration of the secondary submovement to be the amount of time between the end of the primary submovement and the moment at which the overall movement ended. The spatial extent of the secondary submovement corresponded to the net distance traveled during that interval. If one or more of the secondary-submovement criteria were not met, then the overall movement was defined to include only the primary submovement. ${ }^{22}$

An example of the position, velocity, and acceleration records obtained with our movement-parsing algorithm appears in Figure 5. These records are from a representative movement composed of both a primary and secondary submovement for a target region whose distance and width equaled $15.8^{\circ}$ and $6.3^{\circ}$, respectively. The movement beginning (primary-submovement start), end of the primary submovement, and end of the secondary submovement (i.e., overall movement end) are marked at the time points where the parsing algorithm identified them to have occurred.

\section{Results}

Some results of Experiment 1 are summarized in Table 4. For each of the 12 target conditions, this table lists obtained

\footnotetext{
${ }^{20}$ The filters used in steps (a), (b), and (c) were, respectively, the NER, NERD, and MAXFLAT digital filters of Kaiser and Reed (1977). The NER and NERD filters produce outputs with no temporal phase shift and nearly equal ripple in the frequency domain. The MAXFLAT filter produces an output with a maximally flat frequency response and no phase shift. By using these filters, we obtained accurate movement representations in the time and frequency domains below the selected filter cutoffs.

${ }^{21}$ Following the first relative maximum in the velocity of the primary submovement, the acceleration was by definition negative and reflected the amount of decelerative force being applied to the moving handle during that time interval. Our first parsing criterion (positive-to-negative velocity zero-crossing) corresponds to a reversal in the direction of movement, going from a forward (clockwise) to backward (counterclockwise) direction. Our second parsing criterion (negative-to-positive acceleration zero-crossing) corresponds to a relative minimum in velocity while the velocity is still positive, indicating a speedup in the forward direction after a prior slowing down. For a primary-submovement end to qualify under the second criterion, the velocity at this minimum had to be at least $5 \%$ s less than the immediately prior relative maximum in velocity; otherwise the relative minimum in velocity was ignored, and the search to find the primary-submovement end proceeded onward. This additional requirement helped ensure that artifacts (e.g., physiological tremor) would not trigger an inappropriate detection of submovement ends. Furthermore, our third parsing criterion (relative minimum in absolute value of negative acceleration) corresponds to a positive-to-negative zero crossing in "jerk" (i.e., the derivative of acceleration vs. time), which also represents a salient dynamic characteristic (Nelson, 1983). It indicates a relatively abrupt increase of braking (i.e., slowing down) in the forward direction. For a primary-submovement end to qualify under the third criterion, the absolute value of acceleration at this moment had to be at least $100^{\circ} / \mathrm{s}^{2}$ less than the immediately prior relative maximum in the absolute value of negative acceleration. Again, this additional requirement helped ensure that artifacts would not trigger the detection of submovement ends.

${ }^{22}$ After the ends of the primary and secondary submovements had been tentatively identified, some further technical adjustments were made in them. Each secondary-submovement end was repositioned to be at the next later moment in time where either a positive-to-negative or negative-to-positive acceleration zero-crossing occurred. Also, for the primary submovements that ended with a positive-to-negative velocity zero-crossing and were not followed by a secondary submovement, we repositioned their ends to be at the next later moment where a positiveto-negative acceleration zero-crossing occurred. These adjustments yielded submovement ends that corresponded to the resting position around which a spring-loaded object would move cyclically as it undergoes a passive damped oscillation.
} 


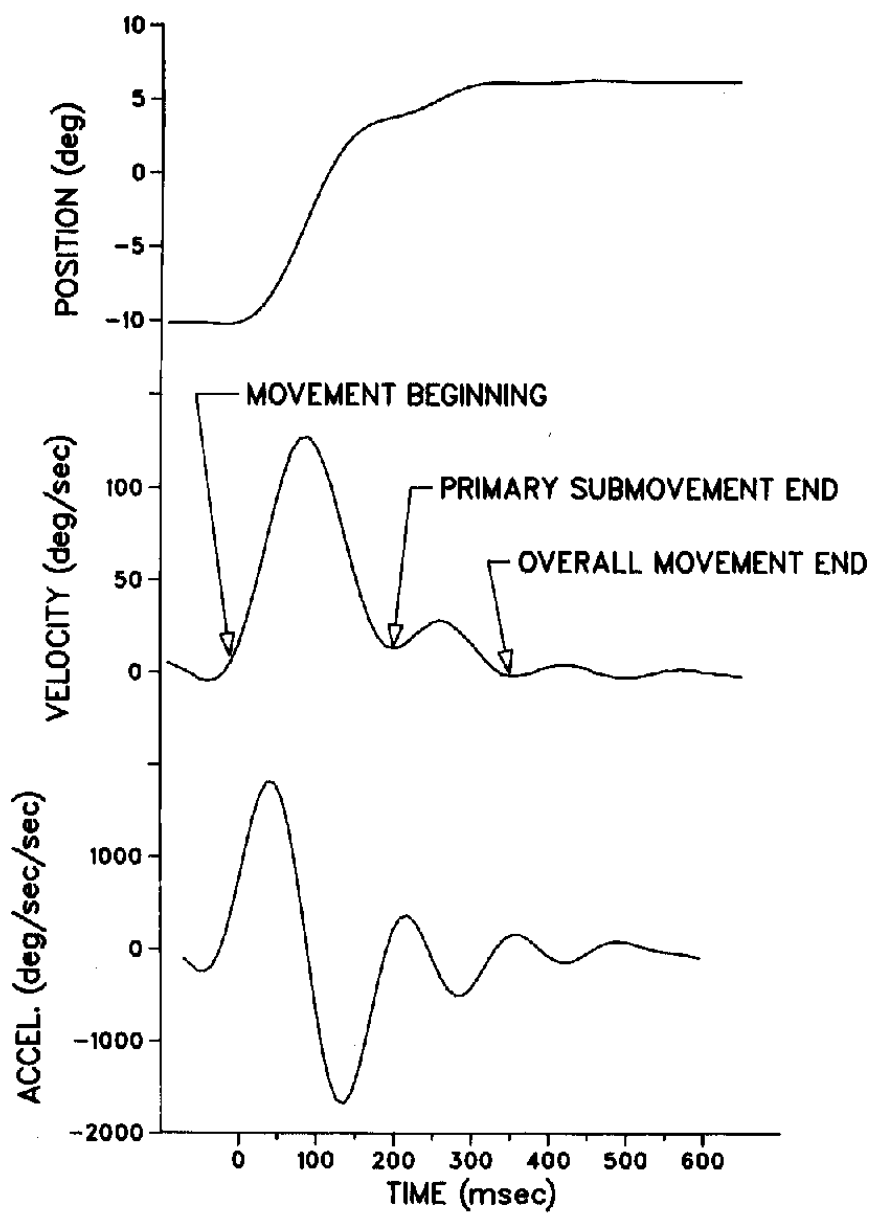

Figure 5. Example of position, velocity, and acceleration records from a representative movement in Experiment 1. (The movement was to a target with distance $D=15.8^{\circ}$ and width $W=6.3^{\circ}$. Arrows indicate the beginning of the movement [i.e., primary-submovement start], end of the primary submovement, and end of the secondary submovement [i.e., overall movement end] as identified by the present movement-parsing algorithm.)

values of six dependent variables: average total movement time $(T)$, mean primary-submovement duration $\left(T_{1}\right)$, mean secondary-submovement duration $\left(T_{2}\right)$, standard deviation of primary-submovement endpoints $\left(S_{1}\right)$, relative frequency of secondary submovements $\left(p_{2}\right)$, and error rate $\left(p_{\mathrm{e}}\right)$. These values were calculated by averaging the data across individual subjects and test sessions.

Noncompensatory distance-width effects. In evaluating the results, we first performed an analysis of variance (ANOVA) on the average total movement times. The purpose of this analysis was to test whether target distance and width had proportionally compensatory effects, as implied by Fitts' law (Equation 1). If such compensation holds, then $T$ should vary directly with $D /$ $W$, and neither $D$ nor $W$ should have any residual effects on $T$ beyond their contributions to the effect of $D / W$. An initial test for compensatory distance-width effects seems appropriate in light of concerns raised by previous investigators (e.g., Sheridan, 1979; Welford, 1968) who have questioned whether $D / W$ fully captures the effects of target distance and width on movement times.
The factors included in the test were the ratio of target distance to width $(D / W)$, target width $(W)$ within each level of $D /$ $W$, and subjects. Two of these factors $(D / W$ and $W)$ were treated as fixed effects and the other (subjects) as a random effect. Replicating previous reports (Keele, 1968, 1981), we found that $D /$ $W$ had a highly significant effect on the average total movement time, $F(4,33)=73.2, p<.001$; as $D / W$ increased, so did $T$. However, variations of target width within the fixed levels of $D /$ $W$ also had significant effects, $F(7,33)=2.72, p<.05$. As $W$ (and $D$ ) increased while $D / W$ remained constant, $T$ tended to increase as well. The latter result means, in essence, that a given multiplicative increase in $W$ did not completely compensate for a corresponding multiplicative increase in $D$; rather, the effect due to increasing $D$ was greater than the effect due to increasing $W$.

Subjective target width. Such noncompensatory distancewidth effects on movement times have also been noted in a few earlier studies (e.g., Sheridan, 1979; Welford, 1968). According to one interpretation, the lack of strict compensation indicates that subjects do not treat the width of the target as being exactly what the experimenter specifies objectively. For example, subjects may act as if relatively narrow targets are wider than they really are and relatively wide targets are narrower than they really are. This would compress the range of subjective target widths compared with the range of objective widths, making changes in objective target width have less effect than they might otherwise. Some investigators have therefore suggested that dependent variables like the average total movement time should be analyzed in terms of subjective rather than objective target width (Welford, 1968). ${ }^{23}$

This suggestion seems reasonable in light of other data from Experiment 1. For example, consider Figure 6. Here we have plotted the standard deviations of secondary-submovement endpoints $\left(S_{2}\right)$ versus objective target width $(W) . S_{2}$ increased significantly as a function of $W, F(3,9)=8.25, p<.01$. However, the best fitting regression line for the data $\left(S_{2}=.336+\right.$ $.128 W ; r=.99)$ had a large positive intercept, rather than exhibiting a directly proportional relation. The lack of direct proportionality implies that subjects did not fully heed the systematic variations in objective target width. The endpoints of secondary submovements toward wide targets were not as dispersed as they could have been without a big sacrifice in accuracy, and the endpoints of secondary submovements toward narrow targets were more dispersed than they should have been relative to those for the wide targets.

Consequently, in further analyses of the movement times and other results from Experiment 1, we incorporated estimates of the subjective width $\left(W_{3}\right)$ for each of the 12 target conditions. ${ }^{24}$

\footnotetext{
${ }^{23}$ The subjective target width has also been called the "effective target width" (Welford, 1968). However, this latter term may have alternative meanings, depending on whether it is used in the context of spatially constrained or temporally constrained movement tasks (Schmidt, Zelaznik, Hawkins, Frank, \& Quinn, 1979). For present purposes, we therefore prefer the term subjective target width.

${ }^{24} \mathrm{We}$ did not make a corresponding distinction between the objective and subjective target distances $\left(D\right.$ vs. $D_{\mathrm{s}}$ ) that subjects used in producing their movements. Such a distinction might be appropriate if subjects systematically aim too short or too long relative to the target center, yielding consistent undershoots or overshoots (constant errors). How-
} 
Table 4

Results of Experiment 1

\begin{tabular}{|c|c|c|c|c|c|c|c|c|}
\hline \multirow[b]{2}{*}{$\begin{array}{c}\text { Width } \\
\text { (degree) }\end{array}$} & \multirow[b]{2}{*}{$\begin{array}{l}\text { Distance } \\
\text { (degree) }\end{array}$} & \multirow{2}{*}{$\begin{array}{l}\text { Subjective } \\
\text { width } \\
\text { (degree) }\end{array}$} & \multicolumn{6}{|c|}{ Dependent variable } \\
\hline & & & $\begin{array}{c}T \\
\text { (milliseconds) }\end{array}$ & $\begin{array}{c}T_{1} \\
\text { (milliseconds) }\end{array}$ & $\begin{array}{c}T_{2} \\
\text { (milliseconds) }\end{array}$ & $\begin{array}{c}S_{1} \\
\text { (degree) }\end{array}$ & $p_{2}(\%)$ & $p_{c}(\%)$ \\
\hline 1.61 & $\begin{array}{l}15.81 \\
25.00\end{array}$ & $\begin{array}{l}2.35 \\
2.01\end{array}$ & $\begin{array}{l}529 \\
583\end{array}$ & $\begin{array}{l}268 \\
309\end{array}$ & $\begin{array}{l}261 \\
274\end{array}$ & $\begin{array}{l}2.46 \\
3.60\end{array}$ & $\begin{array}{l}86.1 \\
91.1\end{array}$ & $\begin{array}{l}17.5 \\
15.6\end{array}$ \\
\hline 2.54 & $\begin{array}{l}10.00 \\
15.81 \\
25.00 \\
39.52\end{array}$ & $\begin{array}{l}3.35 \\
3.02 \\
3.02 \\
3.02\end{array}$ & $\begin{array}{l}338 \\
441 \\
505 \\
583\end{array}$ & $\begin{array}{l}236 \\
277 \\
305 \\
308\end{array}$ & $\begin{array}{l}102 \\
164 \\
200 \\
275\end{array}$ & $\begin{array}{l}1.33 \\
2.36 \\
2.78 \\
5.08\end{array}$ & $\begin{array}{l}49.6 \\
71.9 \\
85.1 \\
93.7\end{array}$ & $\begin{array}{l}15.0 \\
10.4 \\
11.2 \\
12.7\end{array}$ \\
\hline 4.01 & $\begin{array}{l}10.00 \\
15.81 \\
25.00 \\
39.52\end{array}$ & $\begin{array}{l}3.35 \\
4.02 \\
3.35 \\
3.69\end{array}$ & $\begin{array}{l}300 \\
386 \\
449 \\
532\end{array}$ & $\begin{array}{l}225 \\
248 \\
281 \\
316\end{array}$ & $\begin{array}{r}75 \\
138 \\
168 \\
216\end{array}$ & $\begin{array}{l}1.13 \\
2.06 \\
2.49 \\
4.51\end{array}$ & $\begin{array}{l}44.7 \\
69.3 \\
77.1 \\
88.7\end{array}$ & $\begin{array}{l}2.3 \\
5.8 \\
3.5 \\
6.0\end{array}$ \\
\hline 6.34 & $\begin{array}{l}15.81 \\
25.00\end{array}$ & $\begin{array}{l}5.70 \\
5.36\end{array}$ & $\begin{array}{l}321 \\
395\end{array}$ & $\begin{array}{l}229 \\
258\end{array}$ & $\begin{array}{r}92 \\
137\end{array}$ & $\begin{array}{l}2.04 \\
2.76\end{array}$ & $\begin{array}{l}53.0 \\
77.1\end{array}$ & $\begin{array}{l}3.8 \\
2.9\end{array}$ \\
\hline
\end{tabular}

Note. $T=$ average total movement time; $T_{1}=$ mean primary-submovement duration; $T_{2}=$ mean secondary-submovement duration; $S_{1}=$ standard deviation of primary-submovement endpoints; $p_{2}=$ relative frequency of secondary submovements; and $p_{\mathrm{e}}=$ error rate.

The subjective width estimates appear in Table 4. They were derived from an estimation procedure designed to test the predictions of the stochastic optimized-submovement model against observed error rates and frequencies of secondary submovements. The estimated $W_{\mathrm{s}}$ values maximized the model's goodness-of-fit to these data. That the model also happens to fit the associated movement times reasonably well is a fortunate by-product, not a guaranteed outcome, of the estimation procedure. $^{25}$

Average total movement times. Figure 7 shows the average total movement times $(T)$ versus the square root of the target distance divided by the subjective target width for the 12 target conditions. The closed circles in the figure represent the actual data, and the solid regression line represents the square-root function that best fits them $\left(T=103+137 \sqrt{D / W_{s}}\right)$. The fit is reasonably good $(r=.96)$, accounting for almost $92 \%$ of the variance in the average total movement times. This outcome is consistent with the stochastic optimized-submovement model (Equation 4). ${ }^{26}$

Mean primary-submovement durations. Figure 7 (open circles and dashed line) also shows the mean durations of primary submovements. These were not constant across target conditions, contrary to the deterministic iterative-corrections model (cf. Keele, 1968). Instead, they increased significantly with the difficulty of the target, $F(11,33)=8.07, p<.001$. When we regressed the observed values of $T_{1}$ against $\sqrt{D / W_{s}}$, a good fit emerged $\left(T_{1}=160+45 \sqrt{D / W_{s}} ; r=.95\right)$. Again the results are consistent with the stochastic optimized-submovement model (Equation 5).

Standard deviations of primary-submovement endpoints. The stochastic optimized-submovement model is likewise supported by other features of the primary submovements. Accord-

ever, the constant errors observed in the movement endpoints during Experiment 1 were sufficiently small that we disregarded them for present purposes. Incorporating estimates of the subjective target distance would not change our conclusions dramatically. ing to the model (Equation 2), primary submovements should have endpoints whose standard deviations $\left(S_{1}\right)$ in space are directly proportional to the average velocity of the primary submovements (i.e., $D_{1} / T_{1}$, where $D_{1}$ is the mean distance traveled by the primary submovements). We tested this assumption by computing $S_{1}$ and the average primary-submovement velocity for each of the 12 target conditions. These results appear in Figure 8 .

The standard deviations of the primary-submovement endpoints (Figure 8, closed circles) increased significantly with the average velocity of the primary submovements, $F(11,33)=$ $10.9, p<.001$. A linear regression (Figure 8, solid line) accounted for about $66 \%$ of the variance in the data $\left(S_{1}=.02+\right.$ $\left.42\left[D_{1} / T_{1}\right] ; r=.82\right)$. The pattern is similar to those found in studies of temporally constrained movements toward discrete target points (e.g., Schmidt et al., 1978, 1979; Wright, 1983a, 1983b; Wright \& Meyer, 1983; Zelaznik et al., 1981), where endpoint deviations have increased linearly with the average

\footnotetext{
${ }^{25}$ Alternatively, Welford (1968) has suggested that effective target width may be estimated from the standard deviation of the endpoints for all movements made to a specified target region. However, as Figure 6 indicates, we have focused just on the endpoints of the secondary submovements to estimate the subjective target width $\left(W_{\mathrm{s}}\right)$. Movements that included a primary submovement without a secondary submovement are excluded from consideration here. This is because under the stochastic optimized-submovement model, the primary-submovement endpoints would not have standard deviations $\left(S_{1}\right)$ proportional to either the objective or subjective target width. Their standard deviations depend on other factors outlined elsewhere (e.g., Equation 2 and Appendix), even if the secondary-submovement endpoints have standard deviations $\left(S_{2}\right)$ proportional to the subjective target width.

${ }^{26}$ We also regressed the average total movement times $(T)$ against $\log _{2}\left(2 D / W_{\mathrm{s}}\right)$. This provided a direct test of Fitts' law (Equation 1), yielding $T=21+120 \log _{2}\left(2 D / W_{s}\right)$. The regression accounted for about $94 \%$ of the variance in the data. A good fit is to be expected, given that square-root and logarithmic functions of $D / W_{s}$ are both monotonically increasing with negative second derivatives (Footnote 13).
} 


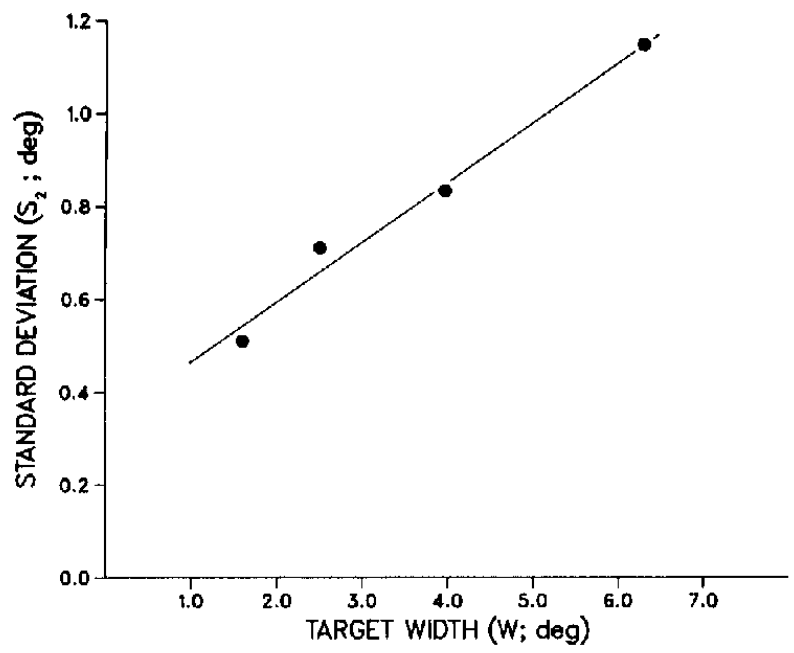

Figure 6. Average standard deviations of secondary-submovement endpoints $\left(S_{2}\right)$ versus objective target width $(W)$ in Experiment 1 . (The closed circles are the data, and the solid line is the best fitting linear regression function.)

movement velocity. This accords well with the stochastic optimized-submovement model, in which the greater forces needed to generate higher velocity movements are supposedly accompanied by amplified neuromotor noise.

Relative frequencies of secondary submovements. The relative frequencies of secondary submovements $\left(p_{2}\right)$ increased with $D / W_{\mathrm{s}}, F(11,33)=11.0, p<.001$. These data support the stochastic optimized-submovement model qualitatively, and they provide an additional basis for testing the model quantitatively. In particular, we have used them as part of fitting Equation 7 from the model. This involved an iterative search for estimates of the parameter $\theta$ that would minimize discrepancies between the predicted and observed values of $p_{2} .{ }^{27}$

The goodness of fit achieved by the search may be assessed in Figure 9, where observed $p_{2}$ values from each target condition

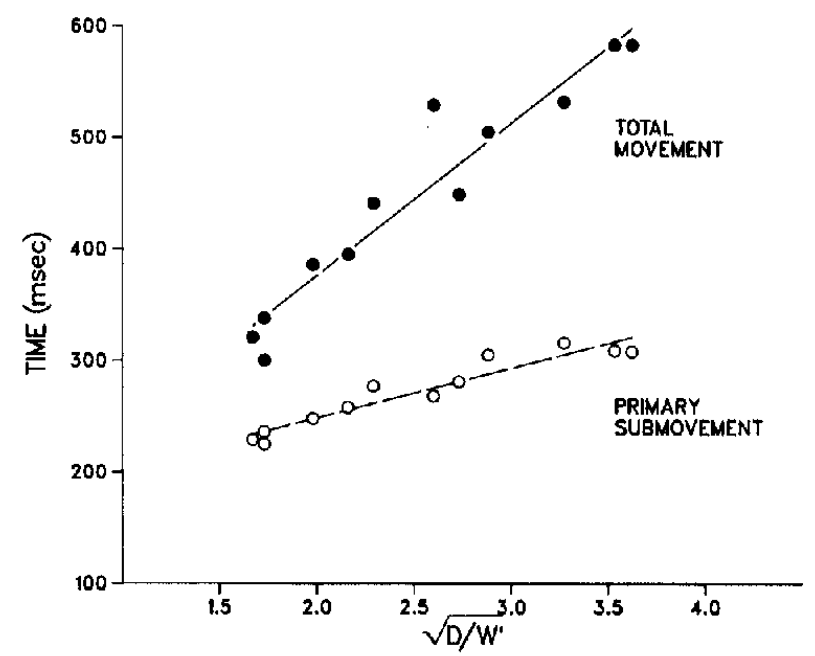

Figure 7. Average total movement times ( $T$; closed circles) and primarysubmovement durations $\left(T_{1}\right.$; open circles) versus $\sqrt{D / W_{\mathrm{s}}}$, for each target of Experiment 1 , where $D=$ objective target distance and $W_{\mathrm{s}}=$ subjective target width. (The solid and dashed lines are best fitting regression functions.)

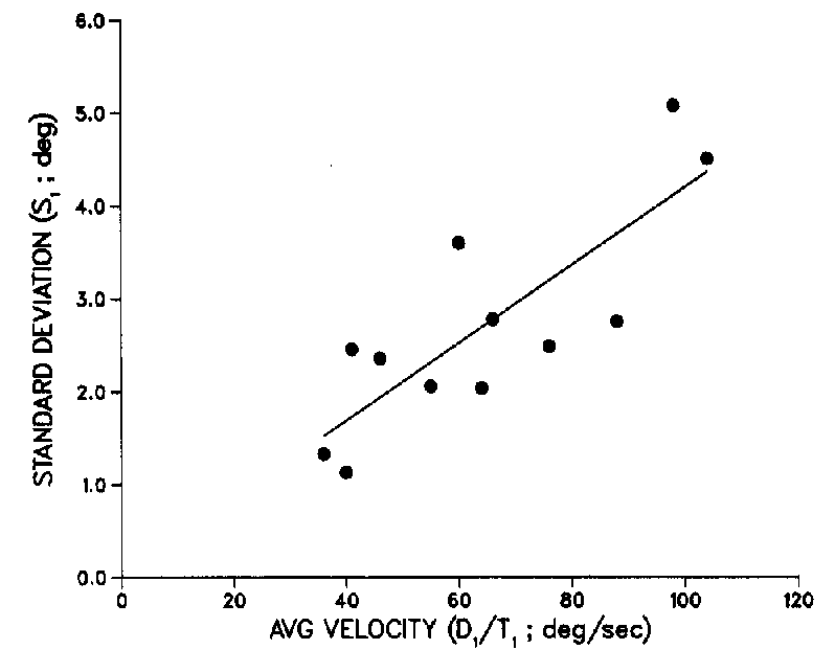

Figure 8. Standard deviations of primary-submovement endpoints $\left(S_{1}\right)$ versus the average velocity of the primary submovements $\left(D_{1} / T_{1}\right)$ for each target of Experiment 1. (The closed circles are the data, and the solid line is the best fitting linear regression function.)

are compared with predicted $p_{2}$ values from the model. The predicted values tended to overestimate the observed values for easy targets (i.e., ones with relatively small $D / W_{\mathrm{s}}$ ) and to underestimate them for difficult targets (i.e., ones with relatively large $\left.D / W_{3}\right)$, as indicated by the closed circles versus dashed line, $\chi^{2}(12)=86.0, p<.01$. Nevertheless, the stochastic optimizedsubmovement model still provides a reasonable qualitative account. A majority of the corresponding observed and predicted values of $p_{2}$ differed by less than $10 \%$ in absolute magnitude, and the correlation between them was highly positive $(r=.96$, $p<.01$ ). We will say more later about what the fairly small, but statistically significant, deviations might imply (see General Discussion).

Mean secondary-submovement durations. The mean durations of secondary submovements increased as target difficulty increased $\left(T_{2}=-56+92 \sqrt{D / W_{\mathrm{s}}} ; r=.91\right)$. Following Equation 8 , this trend represents a systematic change of the differences between the average total movement times and the mean primary-submovement durations (i.e., $T_{2}=T-T_{1}$ ). Also, in accord with the stochastic optimized-submovement model, $T_{2}$ was consistently less than $T_{1}$, and the difference between $T_{2}$ and $T_{1}$ tended to decrease as target difficulty increased (cf. Footnote 18$){ }^{28}$

${ }^{27}$ During this search, we estimated the subjective target width $\left(W_{8}\right)$ and the parameter $c_{2}$, which represents the probability that the secondary submovements ended inside the subjective target regions. The estimation procedure was guided by trying to maximize the goodness of fit of the stochastic optimized-submovement model to both the secondarysubmovement frequencies and the error rates. For the data in Figures 8 and $9, c_{2}$ was estimated to equal 0.91 .

${ }^{28}$ This pattern of support for the model is not merely a consequence of how we defined $T_{2}$ algebraically. In principle, $T_{1}, T_{2}$, and $T$ could all increase with $\sqrt{D / W_{s}}$, and $T_{2}$ could always have a positive value, but at the same time, $T_{2}$ could be greater than $T_{1}$, and $T_{1}-T_{2}$ could remain constant rather than decreasing with $\sqrt{D / W_{\mathrm{s}}}$. For example, suppose that $T_{2}=T_{1}+A_{2}$ for all target conditions, where $T_{1}$ is a square-root function of $D / W_{\mathrm{s}}$ and $A_{2}$ is a positive constant. Then the model would be violated. 
Error rates. Finally, Figure 10 shows observed and predicted error rates obtained for each combination of target distance and width in Experiment 1. Here the term error refers to any submovement series that, as determined by our movement-parsing algorithm, failed to end inside the objective target region. The observed error rates increased reliably as target difficulty increased, $F(11,33)=4.09, p<.01$. We calculated the predicted error rates $\left(p_{\mathrm{e}}\right)$ from Equation 9 of the stochastic optimizedsubmovement model, using the same parameter values as for the relative frequencies of secondary submovements $\left(p_{2}\right)$.

Supporting the model, the predicted and observed error rates came very close to each other (Figure 10, closed circles vs. dashed line). There were no statistically significant deviations, $\chi^{2}(12)<12.0, p>.5$. This happened despite the small but significant deviations found between the predicted and observed relative frequencies of secondary submovements (Figure 9).

\section{Discussion}

Taken as a whole, the results from Experiment 1 are encouraging for the stochastic optimized-submovement model. We found that the average total times $(T)$, mean primary-submovement durations $\left(T_{1}\right)$, standard deviations of primary-submovement endpoints $\left(S_{1}\right)$, relative frequencies of secondary submovements $\left(p_{2}\right)$, mean secondary-submovement durations $\left(T_{2}\right)$, and error rates $\left(p_{\mathrm{e}}\right)$ all increased with target difficulty $(D /$ $W_{\mathrm{s}}$ ) as the model predicts. In most cases, the quantitative as well as qualitative fit was good. The evidence against the classical deterministic iterative-corrections model (Crossman \& Goodeve, 1963/1983; Keele, 1968), which cannot deal with these results, is quite strong.

The success of the stochastic optimized-submovement model may be attributed to how subjects cope with noise in the neuromotor system. It appears that this noise produces inherent movement variability for which subjects compensate by adopting a nearly ideal compromise between the durations of pri-

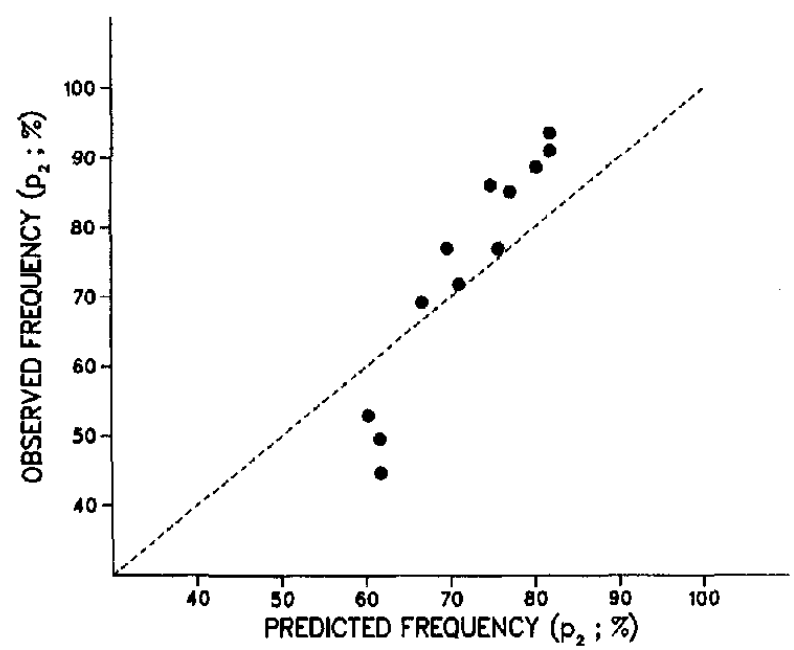

Figure 9. Relative frequencies of secondary submovements $\left(p_{2}\right)$ in Experiment 1 . (The closed circles represent pairs of observed and predicted values for each target, and the dashed diagonal line represents the ideal function that would apply if the predictions of the stochastic optimized-submovement model fit the observations perfectly.)

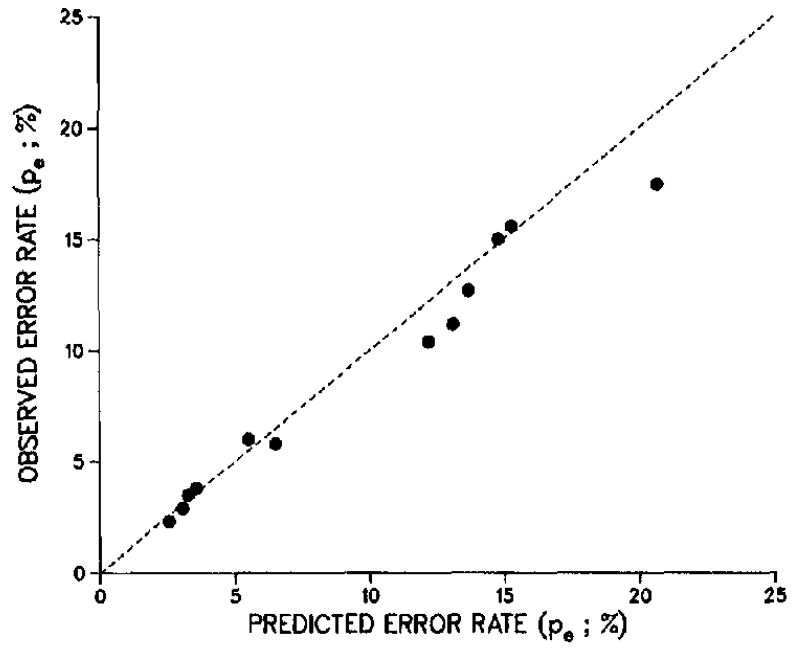

Figure 10. Observed versus predicted error rates (closed circles) for each target of Experiment 1. (The dashed diagonal line represents the ideal function that would apply if the predictions of the stochastic optimizedsubmovement model fit the observations perfectly.)

mary and secondary submovements. In Experiment 2, we further demonstrate how hypotheses concerning the optimality of human motor performance can yield a better understanding of rapid spatially constrained movements and the speed-accuracy trade-offs associated with them.

\section{Experiment 2}

The purpose of Experiment 2 was to test the stochastic optimized-submovement model with respect to spatially constrained movements made in the absence of concurrent visual feedback. According to the model, eliminating such feedback should increase average total movement times or overall error rates (target misses). This follows from the model's assumptions about the nature and purpose of secondary submovements. Secondary submovements are assumed to overcome deviations caused by neuromotor noise in producing the primary submovements, and secondary submovements are assumed to be prepared "on the fly" as the primary submovements progress from start to finish. It is therefore important for the process that produces the secondary submovements to have accurate feedback or feedforward regarding the progress of the primary submovements. If this information is withheld, then the secondary submovements should necessarily suffer in some way.

Confronted by a situation involving visual-feedback deprivation, subjects might adopt either of two strategies. One possibility would entail making primary submovements more slowly and accurately than when full feedback is available, so that the primary submovements still usually end inside the target region. With this strategy, subjects could avoid the need for secondary submovements, thereby precluding the increased error rate that would otherwise accrue as a result of feedback deprivation. However, there would also be an associated cost. The average total movement times would increase above the normal level possible with full feedback, because relatively fast and inaccurate primary submovements must be combined with accu- 
rate secondary submovements to achieve this level while maintaining a set high frequency of target hits.

Another possibility would entail using the two-submovement strategy of the stochastic optimized-submovement model despite an absence of visual feedback about the progress of the primary submovements. With this strategy, subjects could prepare and execute their primary submovements in the usual fashion (Equation 5), supplementing them by attempted secondary corrective submovements, even though visual feedback is absent or degraded. A two-submovement strategy might still be reasonable, for example, if kinesthetic feedback provides a modicum of information about the progress of the primary submovements. In this case, the average total movement times would not necessarily be increased above their normal minimum level, because the durations of the primary submovements would not be increased. However, if the eliminated feedback is truly useful, then visual-feedback deprivation should increase the overall error rate significantly, because the secondary submovements would have to proceed on the basis of relatively degraded information. Indeed, there is already considerable evidence that eliminating visual feedback disrupts the accuracy of rapid spatially constrained movements and amplifies the effects of target difficulty $(D / W)$ on error rates but does not markedly increase average total movement times (Abrams et al., 1983; Wallace \& Newell, 1983). This suggests that when visual feedback is withdrawn, subjects may adhere to the two-submovement strategy instead of adopting a one-submovement strategy and producing their primary submovements more slowly to maintain a low error rate.

Assuming a two-submovement strategy under conditions of feedback deprivation, the stochastic optimized-submovement model makes several related predictions. The mean durations of primary submovements $\left(T_{1}\right)$, as well as the average total movement times $(T)$, should still be a quasi square-root function of $D / W$, satisfying Equations 4 and 5. Moreover, the endpoints of the primary submovements should still have standard deviations $\left(S_{1}\right)$ that increase linearly with the average primarysubmovement velocity $\left(D_{1} / T_{1}\right)$, satisfying Equation 2, and the frequency of target misses by the primary submovements (i.e., $1-c_{1}$ ) should still increase monotonically with $D / W$ (Equation 7). We would not expect feedback deprivation to change the properties of neuromotor noise, because the noise is not perceptual in nature. These expectations may be checked by supplementing the method of Experiment 1 with a manipulation of the visual feedback that subjects receive while performing rapid spatially constrained movements.

\section{Method}

Subjects. Four right-handed University of Michigan students served individually as paid subjects. The subjects had not been in Experiment 1 , but they came from the same population as before. They received wages of $\$ 4$ per hour plus a bonus based on good performance.

Apparatus and movement parsing. The apparatus and algorithm used to parse the movements were the same as in Experiment 1.

Design. The design was similar to that of Experiment 1. Each subject participated for six sessions of 12 blocks each. There were 16 trials per block, including 4 warm-up trials followed by 12 test trials. One combination of target width and distance was assigned to each block. Across blocks, the targets came from the same set of 12 distance-width combinations as before (Table 3 ). The first two sessions provided practice, and the last four served as test sessions. Only data from the test sessions are reported.

Within each block, we included two levels of visual feedback. One of these involved a visible-cursor condition during which subjects could see the display cursor throughout their entire movements. The other involved an invisible-cursor condition during which the cursor was removed from the display screen as soon as the movements started. These conditions alternated systematically back and forth from one trial to the next, so subjects always knew which condition would occur on the next trial. An alternating sequence was used to avoid possible artifacts associated with visual-feedback deprivation (Zelaznik, Hawkins, \& Kisselburgh, 1983). ${ }^{29}$

Procedure. At the beginning of each trial, before using the handle to align the cursor with the fixation dot, the subject verified which of the two cursor conditions would occur on that trial. This involved rotating the handle from side to side so as to pass the cursor over the region of the display screen between the fixation dot and target region. During the preliminary handle rotation, the cursor moved horizontally across the screen as the handle rotated, but the cursor was visible only in the areas where it would be seen during the subsequent test movement (e.g., before trials of the invisible-cursor condition, the cursor could be seen only when it was no more than $1^{\circ}$ to the right of the fixation dot). After verifying the cursor condition, the subject proceeded by aligning the cursor with the fixation dot, as described earlier (Experiment 1).

Successful alignment of the cursor with the fixation dot was followed by the response signal, which cued the subject's movement to the target region. During this movement, the visual feedback for the subject depended on the cursor condition. In the visible-cursor condition, the cursor always remained on the screen and moved continuously as the handle rotated. Thus, the subject had complete visual feedback regarding the relative position of the handle with respect to the target. In the invisible-cursor condition, the cursor disappeared from view as soon as the handle moved $.3^{\circ}$ of clockwise wrist arc from the initial home position toward the target, and the cursor did not reappear until after the movement ended. Because subjects could not see the cursor or the handle, they had no visual feedback for making on-line corrective submovements.

Other aspects of the procedure were similar to those in Experiment 1. The display of the fixation dot and target region remained visible throughout each trial regardless of the cursor condition. Also, at the end of each trial, subjects always received a visual display of the cursor and target, showing them where their movements ultimately stopped relative to the target. Thus, they had the same final knowledge of results under both cursor conditions.

Data analysis. The data from Experiment 2 were analyzed in the same way as those from Experiment 1. In what follows, we first consider subjects' performance under the visible-cursor condition, which provided full visual feedback throughout each trial. Next their performance is considered under the invisible-cursor condition, in which movements were made without concurrent visual feedback.

\section{Results: Visible-Cursor Condition}

Some results obtained under the visible-cursor condition of Experiment 2 are summarized in Table 5. Paralleling our previ-

\footnotetext{
${ }^{29}$ We wanted subjects to be prepared for the feedback and to take full advantage of it when the cursor was visible. On the other hand, we did not want performance to deteriorate too much when visual feedback was unavailable, as might happen if the invisible-cursor condition were run separately from the visible-cursor condition. The alternating trial sequence permitted a reasonable compromise in achieving these disparate goals. For example, it helped distribute any general practice effects evenly across the two cursor conditions.
} 
Table 5

Results of Experiment 2: Visible-Cursor Condition

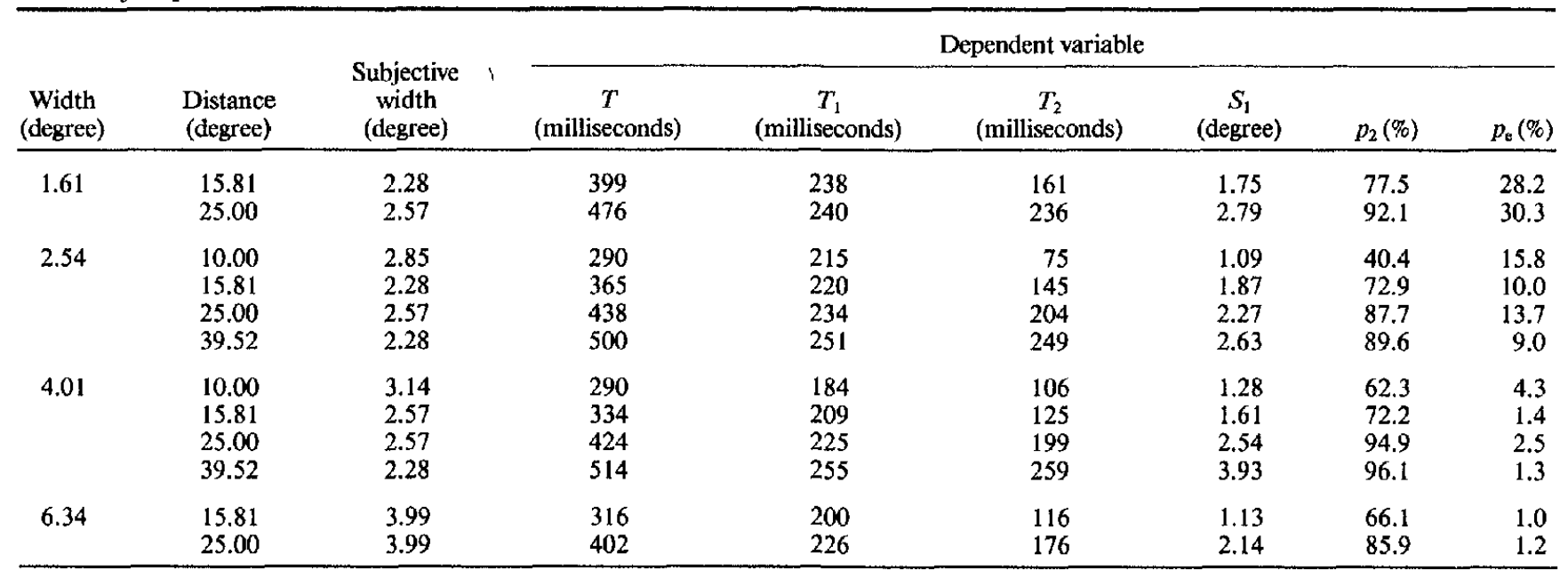

Note. $T=$ average total movement time; $T_{1}=$ mean primary-submovement duration; $T_{2}=$ mean secondary-submovement duration; $S_{1}=$ standard deviation of primary-submovement endpoints; $p_{2}=$ relative frequency of secondary submovements; and $p_{e}=$ error rate.

ous analyses (cf. Results, Experiment 1), this table outlines several relevant dependent variables as a function of target distance and width. In each case, there is some additional support for the stochastic optimized-submovement model.

Noncompensatory distance-width effects. We again began by testing whether the objective target distance and width had proportionally compensatory effects on the average total movement times $(T)$ when there was full visual feedback. This involved another ANOVA whose factors included the different levels of $D / W$, the levels of $W$ within each $D / W$ level, and subjects. The $D / W$ ratio affected $T$ significantly, $F(4,33)=45.9, p<$ .001 , replicating Experiment 1 . As before, there were also significant effects of target width within the $D / W$ levels, $F(7$, 33) $=7.86, p<.001$. Movement times tended to increase as $W$ (and $D$ ) increased while $D / W$ remained constant. A given proportional change in $W$ did not completely compensate for the effect of a corresponding proportional change in $D$. Following our previous theoretical interpretation, the lack of compensatory distance-width effects may be attributed to systematic differences between the subjective and objective target widths.

Subjective target width. The contribution of subjective target width to movement performance is illustrated further in Figure 11. Here we have plotted standard deviations of the secondarysubmovement endpoints as a function of the objective target width for the visible-cursor condition (Figure 11, closed circles), taking the same approach as used earlier (cf. Figure 6). A positive linear relation held between these two variables (Figure 11 , solid line; $S_{2}=.52+.056 W ; r=.97$ ), but the relation was not a directly proportional one. $S_{2}$ was relatively large for narrow targets and relatively small for wide targets compared with what would have happened given direct proportionality. As in Experiment 1, it appears that subjects treated the narrow targets as being wider than they really were and the wide targets as being narrower than they really were. Consequently, in further analyses of the data, we used estimates of subjective target width $\left(W_{s}\right)$ rather than objective width for assessing movement performance and testing the predictions of the stochastic optimized- submovement model. The $W_{\mathrm{s}}$ estimates appear in Table 5 and were obtained in the same manner as described earlier (see $R e$ sults, Experiment 1).

Average total movement times. Our analyses revealed that when the cursor was visible, the average total movement times had a strong positive relation with target difficulty $(T=131+$ $\left.95 \sqrt{D / W_{s}} ; r=.95\right)$. This relation accounted for over $90 \%$ of the variance in the data, consistent with Equation 4 of the stochastic optimized-submovement model. ${ }^{30}$ The pattern of results, which appears in Figure 12 (closed circles and solid line), was analogous to the one obtained in Experiment 1 (cf. Figure 7).

Mean primary-submovement durations. The mean durations of primary submovements under the visible-cursor condition (Figure 12, open circles) increased significantly as the target difficulty increased, $F(11,33)=3.01, p<.01$, supporting the stochastic optimized-submovement model further. A squareroot trade-off function conformed reasonably well to these data $\left(T_{1}=160+23 \sqrt{D / W_{s}} ; r=.88\right)$. The pattern of results substantiates our hypothesis that adjustments of primary-submovement durations contribute to subjects' strategy for coping with the effects of neuromotor noise and for minimizing the average total movement times. Contrary to the deterministic iterativecorrections model (Crossman \& Goodeve, 1963/1983; Keele, 1968), the primary submovements did not have constant mean durations.

Standard deviations of primary-submovement endpoints. As Figure 13 indicates, the stochastic optimized-submovement model is likewise supported by the standard deviations of the primary-submovement endpoints $\left(S_{1}\right)$ observed in the visiblecursor condition. We again found that these deviations increased significantly with the average velocity of the primary

\footnotetext{
${ }^{30}$ The importance of using the subjective rather than objective target width as a predictor variable may be appreciated by regressing $T$ against $\sqrt{D / W}$ in Table 5. This latter regression yields $r^{2}=.76$, which is considerably less than the amount of variance for which $\sqrt{D / W_{8}}$ can account.
} 


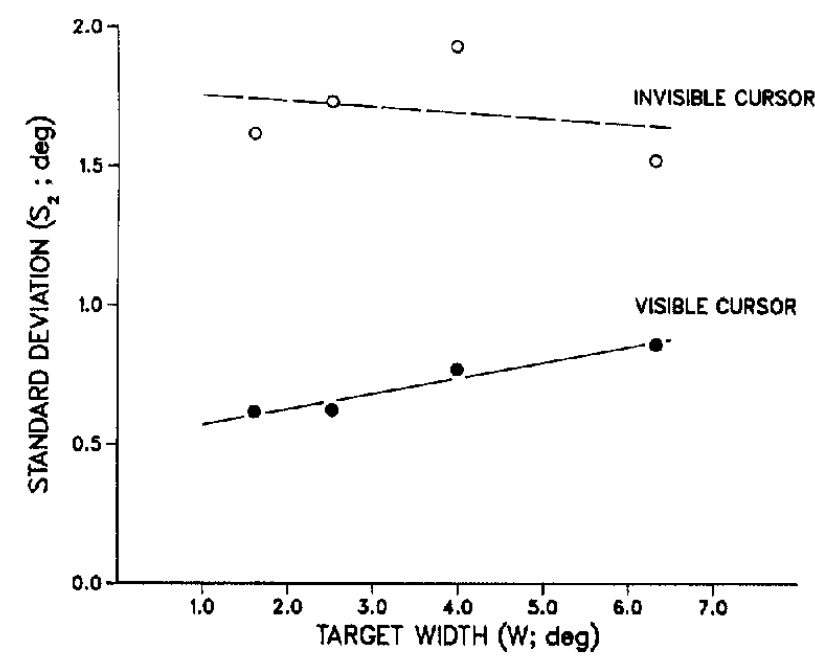

Figure 11. Average standard deviations of secondary-submovement endpoints $\left(S_{2}\right)$ versus objective target width $(W)$ in Experiment 2. (The closed circles and solid line are respectively the data and best-fitting regression function for the visible-cursor condition. The open circles and dashed line are respectively the data and best-fitting regression function for the invisible-cursor condition.)

submovements, $F(11,33)=3.76, p<.01$, and they were roughly linear with the average velocity $\left(S_{1}=.17+22\left[D_{1} / T_{1}\right]\right.$; $r=.82$ ). This is consistent with Equation 2, which stems from the model's assumptions about the effects of neuromotor noise on the variability of underlying force pulses.

Relative frequencies of secondary submovements. Because of subjects' attempts to deal with the effects of neuromotor noise, the relative frequencies of secondary submovements $\left(p_{2}\right)$ increased with target difficulty in the visible-cursor condition (Table 5), $F(11,33)=4.70, p<.01$. When we fit the predictions of the stochastic optimized-submovement model (Equation 7) to the data, some statistically significant deviations again emerged, $\chi^{2}(12)=72.3, p<.01$. The observed values of $p_{2}$ for easy targets were less than predicted, whereas the observed values of $p_{2}$ for difficult targets were greater than predicted (Figure 14, closed circles vs. solid line). However, there was a strong positive correlation between the observed and predicted values $(r=.88, p<$ .01 ). Most of them differed by less than $10 \%$ in absolute magnitude, and the model accounted well for the data qualitatively.

Mean secondary-submovement durations. Applying Equation 8 , we again calculated the mean durations of secondary submovements $\left(T_{2}\right)$ by subtracting the mean primary-submovement durations $\left(T_{1}\right)$ from the average total movement times $(T)$. This yielded a square-root function of $D / W_{\mathrm{s}}$, further supporting the stochastic optimized-submovement model $\left(T_{2}=\right.$ $\left.-29+71 \sqrt{D / W_{\mathrm{s}}} ; r=.94\right)$. Also, in accord with the model, $T_{2}$ was consistently less than $T_{1}$, and the difference between $T_{2}$ and $T_{1}$ tended to decrease as target difficulty increased (cf. Footnote 28).

Error rates. The stochastic optimized-submovement model provides a good account for error rates in the visible-cursor condition of Experiment 2, just as it did in Experiment 1 (cf. Figure 10). More errors occurred as target difficulty increased, $F(11$, $33)=8.96, p<.001$. When we fit the predictions of the model (Equation 9) to the data, no significant deviations emerged,

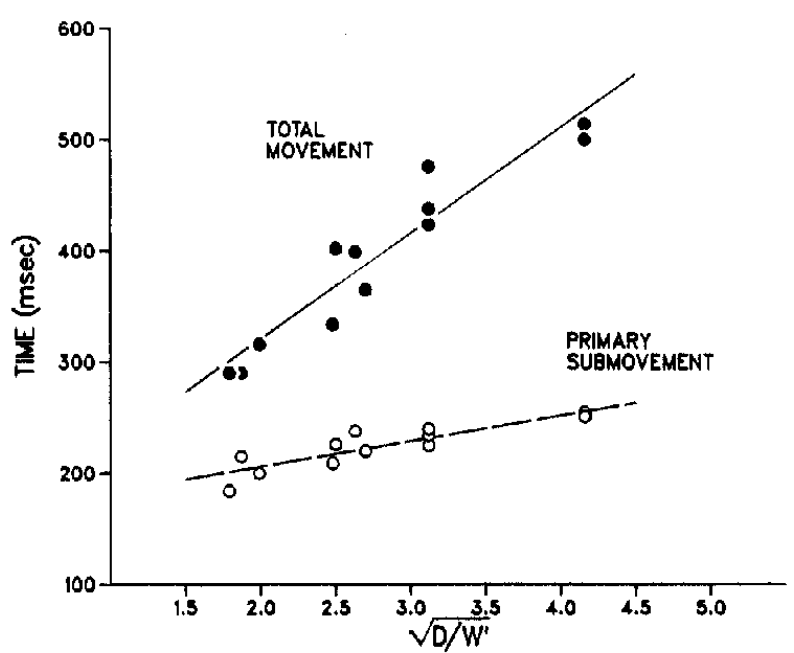

Figure 12. Average total movement times ( $T$; closed circles) and primary-submovement durations ( $T_{1}$; open circles) versus $\sqrt{D / W_{s}}$ for each target of Experiment 2 in the visible-cursor condition. (The solid and dashed lines are best fitting regression functions.)

$\left.\chi^{2}(12)<12.0, p\right\rangle .50$. The goodness of fit is illustrated in Figure 15, where the correspondence between predicted and observed values (closed circles) may be compared against what would have happened if the model fit the data perfectly (dashed diagonal line).

\section{Results: Invisible-Cursor Condition}

Some results obtained under the invisible-cursor condition of Experiment 2 are summarized in Table 6 . We will discuss this table according to the same format as for the visible-cursor con-

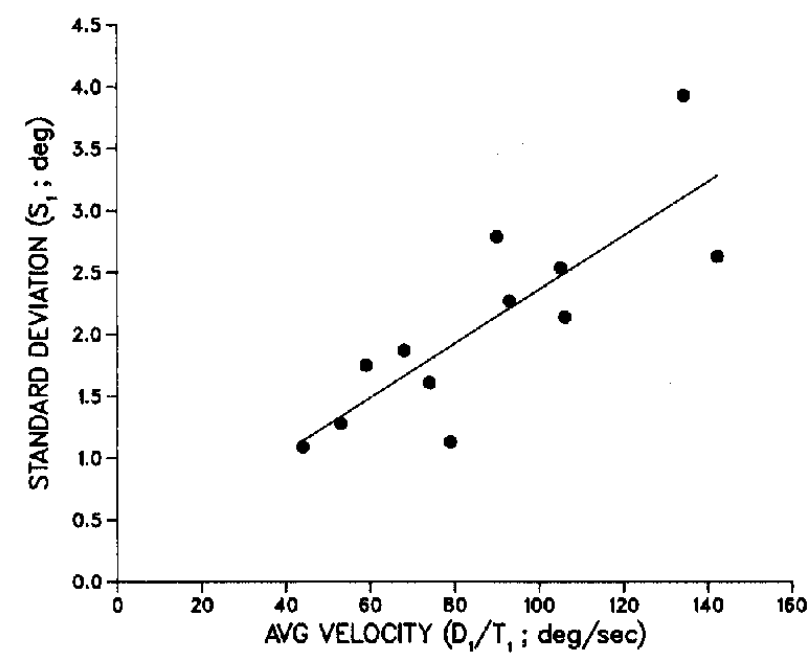

Figure 13. Standard deviations of primary-submovement endpoints $\left(S_{1}\right)$ versus the average velocity of the primary submovements $\left(D_{1} / T_{1}\right)$ for each target of Experiment 2 in the visible-cursor condition. (The closed circles are the data, and the solid line is the best fitting linear regression function.) 


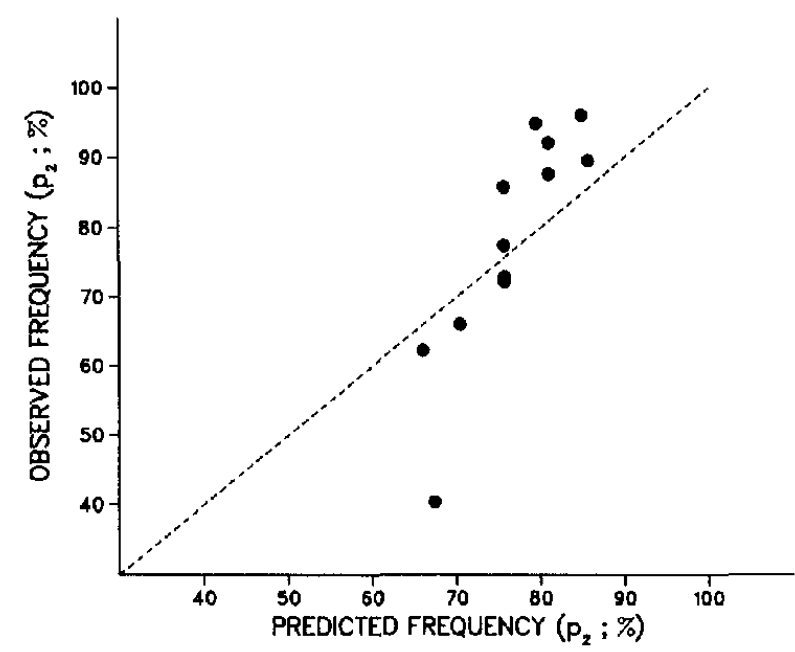

Figure 14. Relative frequencies of secondary submovements $\left(p_{2}\right)$ in the visible-cursor condition of Experiment 2. (The closed circles represent pairs of observed and predicted values for each target, and the dashed diagonal line represents the ideal function that would apply if the predictions of the stochastic optimized-submovement model fit the observations perfectly.)

dition, noting points of similarity and difference owing to the effects of visual-feedback deprivation (cf. Table 5).

Noncompensatory distance-width effects. Like the visiblecursor condition, the invisible-cursor condition yielded noncompensatory effects of objective target distance and width on average total movement times. The $D / W$ ratio affected $T$ significantly when the cursor was invisible, $F(11,33)=12.4, p<$ .001 , paralleling our previous findings. Once again, however, there were also significant effects of $W$ within the individual $D /$ $W$ levels, $F(7,33)=4.38, p<.01$. $T$ tended to increase as $W$ (and $D$ ) increased while $D / W$ remained constant, implying that a given proportional change in $W$ did not completely compensate for the effect of a corresponding proportional change in $D$. This suggests that the subjective target width did not equal the objective width, just as under the visible-cursor condition.

Subjective target width. Because of these noncompensatory distance-width effects, we have analyzed the remaining results from the invisible-cursor condition in terms of subjective rather than objective target widths. The values of $W_{\mathrm{s}}$ used here were the same as those used for the visible-cursor condition (cf. Table 5 ). We did not estimate $W_{s}$ separately when the cursor was invisible, so as outlined subsequently, it is encouraging that the stochastic optimized-submovement model continued to fit most of the data reasonably well.

Average total movement times. For example, consider Figure 16. Here the average total movement times (closed circles) for the invisible-cursor condition have been plotted versus $\sqrt{D / W_{\mathrm{s}}}$. A square-root trade-off function (solid line; $T=204+$ $60 \sqrt{D / W_{\mathrm{s}}} ; r=.93$ ) accounts for close to $90 \%$ of the variance in these data, $F(1,33)=2.84, p<.10$, consistent with Equation 4 of the stochastic optimized-submovement model. This outcome also confirms results of previous investigators who have found that durations of rapid spatially constrained movements approximate Fitts' law even when visual feedback is absent (Prablanc et al., 1979; Wallace \& Newell, 1983).
There were quantitative differences, however, in the average total movement times for the invisible-cursor condition compared with those for the visible-cursor condition (cf. Figure 12, closed circles and solid line). Eliminating the cursor during subjects' movements increased the intercept $(A)$ and decreased the slope $(B)$ of the trade-off between $T$ and $\sqrt{D / W_{\mathrm{s}}}$. We will show that these changes were localized in the secondary submovements and that the primary submovements remained relatively unchanged when visual feedback was absent.

Mean primary-submovement durations. The mean durations of primary submovements under the invisible-cursor condition appear in Figure 16 (open circles and dashed line) as a function of $\sqrt{D / W_{\mathrm{s}}}$. A square-root trade-off fit these data just as well as it did for the mean primary-submovement durations under the visible-cursor condition $\left(T_{1}=158+24 \sqrt{D / W_{s}} ; r=.92\right)$. The slope and intercept of this function each differed by only a few milliseconds from what obtained when visual feedback was present (cf. Figure 12, open circles and dashed line). There is no evidence here that visual-feedback deprivation significantly altered subjects' strategy for preparing and producing the primary submovements.

Standard deviations of primary-submovement endpoints. This result is complemented by the standard deviations of the primary-submovement endpoints under the invisible-cursor condition, which appear in Figure 17. Here $S_{1}$ increased linearly with the average primary-submovement velocity, $F(11$, $33)=5.21, p<.01$, just as it did under the visible-cursor condition. The linear regression function accounted for $84 \%$ of the variance when the cursor was invisible $\left(S_{1}=.03+27\left[D_{1} / T_{1}\right]\right.$; $r=.92$ ), further supporting the stochastic optimized-submovement model's assumptions about the effects of neuromotor noise on endpoint variability (Equation 2). The parameters (slope and intercept) of this function were similar to those obtained when the cursor was visible (cf. Figure 13).

Relative frequencies of secondary submovements. Moreover, despite the absence of concurrent visual feedback, the stochas-

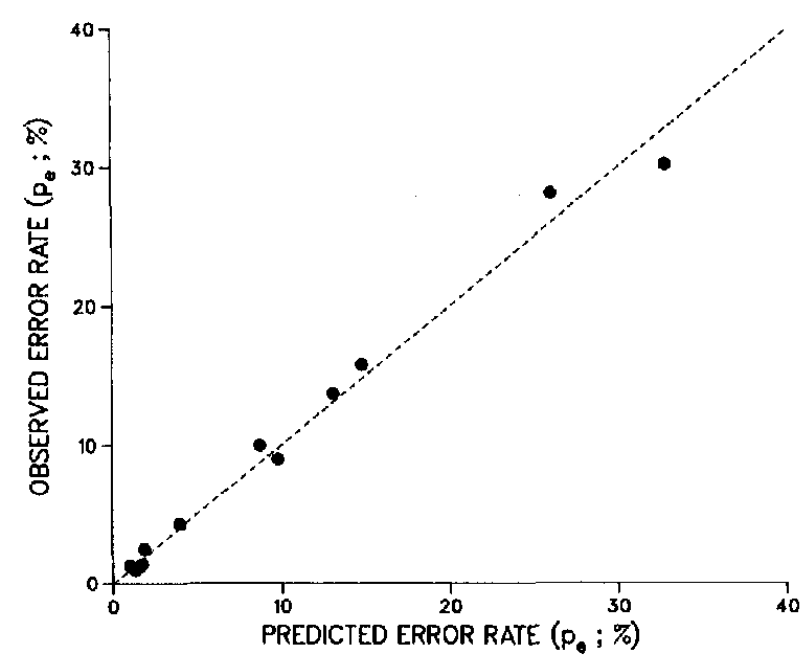

Figure 15. Observed versus predicted error rates (closed circles) for each target in the visible-cursor condition of Experiment 2. (The dashed diagonal line represents the ideal function that would apply if the predictions of the stochastic optimized-submovement model fit the observations perfectly.) 
Table 6

Results of Experiment 2: Invisible-Cursor Condition

\begin{tabular}{|c|c|c|c|c|c|c|c|c|}
\hline $\begin{array}{c}\text { Width } \\
\text { (degree) }\end{array}$ & $\begin{array}{c}\text { Distance } \\
\text { (degree) }\end{array}$ & $\begin{array}{c}\text { Subjective } \\
\text { width } \\
\text { (degree) }\end{array}$ & \multicolumn{6}{|c|}{ Dependent variable } \\
\hline 1.61 & $\begin{array}{l}15.81 \\
25.00\end{array}$ & $\begin{array}{l}2.28 \\
2.57\end{array}$ & $\begin{array}{l}369 \\
400\end{array}$ & $\begin{array}{l}227 \\
249\end{array}$ & $\begin{array}{l}142 \\
151\end{array}$ & $\begin{array}{l}1.72 \\
3.09\end{array}$ & $\begin{array}{l}75.0 \\
79.7\end{array}$ & $\begin{array}{l}50.0 \\
69.7\end{array}$ \\
\hline 2.54 & $\begin{array}{l}10.00 \\
15.81 \\
25.00 \\
39.52\end{array}$ & $\begin{array}{l}2.85 \\
2.28 \\
2.57 \\
2.28\end{array}$ & $\begin{array}{l}297 \\
336 \\
407 \\
432\end{array}$ & $\begin{array}{l}207 \\
218 \\
244 \\
246\end{array}$ & $\begin{array}{r}90 \\
118 \\
163 \\
186\end{array}$ & $\begin{array}{l}1.51 \\
1.92 \\
3.36 \\
3.70\end{array}$ & $\begin{array}{l}48.4 \\
67.6 \\
80.6 \\
89.7\end{array}$ & $\begin{array}{l}43.5 \\
44.1 \\
58.2 \\
46.6\end{array}$ \\
\hline 4.01 & $\begin{array}{l}10.00 \\
15.81 \\
25.00 \\
39.52\end{array}$ & $\begin{array}{l}3.14 \\
2.57 \\
2.57 \\
2.28\end{array}$ & $\begin{array}{l}291 \\
376 \\
382 \\
457\end{array}$ & $\begin{array}{l}195 \\
208 \\
232 \\
263\end{array}$ & $\begin{array}{r}96 \\
168 \\
150 \\
194\end{array}$ & $\begin{array}{l}1.53 \\
1.85 \\
3.35 \\
4.30\end{array}$ & $\begin{array}{l}58.0 \\
82.5 \\
83.6 \\
91.7\end{array}$ & $\begin{array}{l}11.6 \\
29.6 \\
41.8 \\
51.7\end{array}$ \\
\hline 6.34 & $\begin{array}{l}15.81 \\
25.00\end{array}$ & $\begin{array}{l}3.99 \\
3.99\end{array}$ & $\begin{array}{l}330 \\
384\end{array}$ & $\begin{array}{l}205 \\
222\end{array}$ & $\begin{array}{l}125 \\
162\end{array}$ & $\begin{array}{l}1.40 \\
3.14\end{array}$ & $\begin{array}{l}73.2 \\
82.6\end{array}$ & $\begin{array}{r}1.8 \\
23.2\end{array}$ \\
\hline
\end{tabular}

Note. $T=$ average total movement time; $T_{1}=$ mean primary-submovement duration; $T_{2}=$ mean secondary-submovement duration; $S_{1}=$ standard deviation of primary-submovement endpoints; $p_{2}=$ relative frequency of secondary submovements; and $p_{\mathrm{e}}=$ error rate.

tic optimized-submovement model still accounts reasonably well for the relative frequencies of secondary submovements $\left(p_{2}\right)$. As Figure 18 reveals, significantly more secondary submovements occurred in response to difficult targets than in response to easy targets under the invisible-cursor condition, $F(11,33)=2.19, p<.05$, consistent with Equation 7 . The secondary submovements tended to have roughly the same relative frequencies regardless of the cursor condition (i.e., a mean of $24 \%$ under the invisible-cursor condition and a mean of $22 \%$ under the visible-cursor condition). Although quantitative deviations of the data from the model's predictions were statistically significant, $\chi^{2}(12)=34.3, p<.01$, most of the observed and predicted $p_{2}$ values differed by less than $10 \%$ in absolute magni-

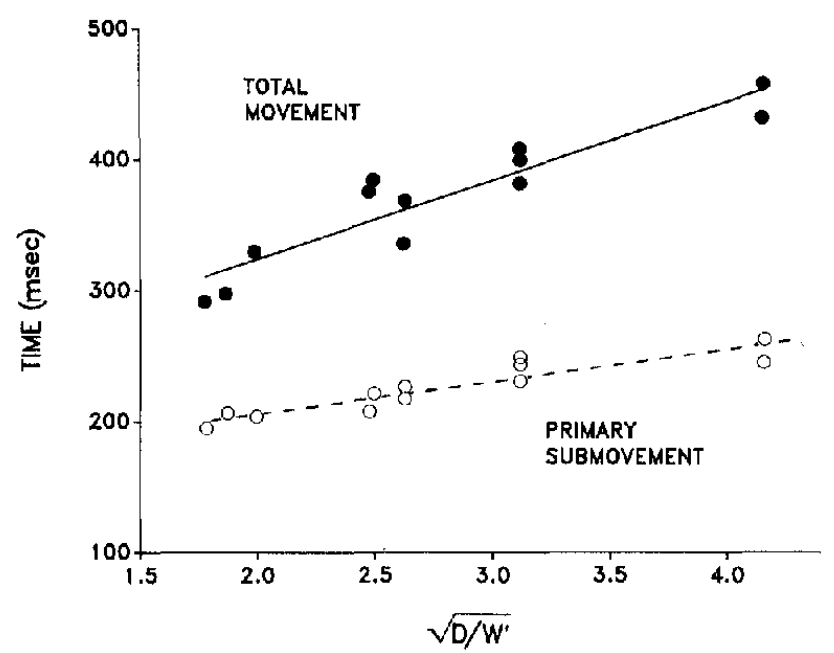

Figure 16. Average total movement times ( $T$; closed circles) and primary-submovement durations $\left(T_{1}\right.$; open circles) versus $\sqrt{D / W_{\mathrm{s}}}$ for each target of Experiment 2 in the invisible-cursor condition. (The solid and dashed lines are best fitting regression functions.) tude when the cursor was invisible (Figure 18, closed circles vs. dashed line).

Mean secondary-submovement durations. Nevertheless, other results of Experiment 2 indicate that visual-feedback deprivation strongly affected some aspects of subjects' secondary submovements. Consider the mean secondary-submovement durations $\left(T_{2}\right)$ from the invisible-cursor condition in Table 6. A square-root trade-off fit these data at least moderately well $(r=.85)$, consistent with the stochastic optimized-submovement model. However, the slope and intercept of the best fitting function were significantly different from those found when the cursor was visible (for the invisible-cursor condition, $T_{2}=46+$

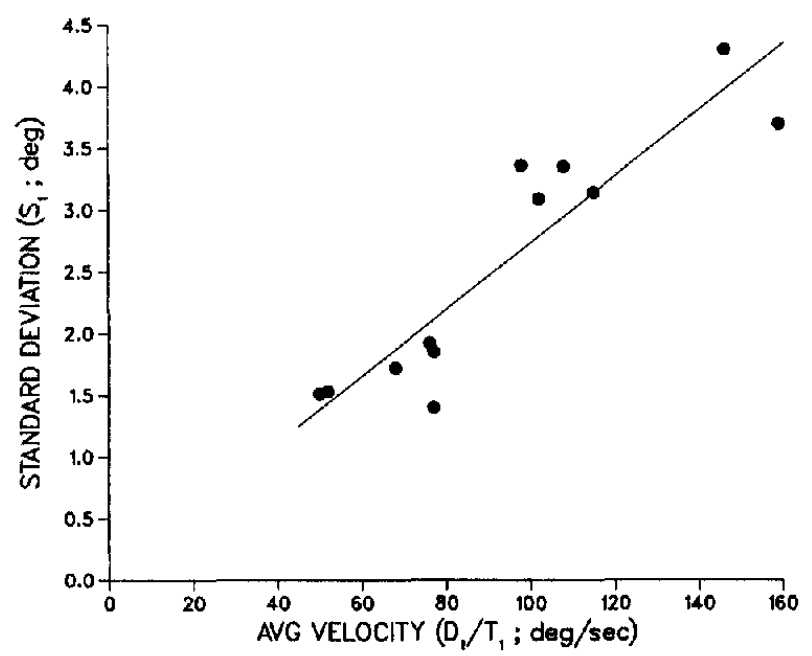

Figure 17. Standard deviations of primary-submovement endpoints $\left(S_{1}\right)$ versus the average velocity of the primary submovements $\left(D_{1} / T_{1}\right)$ for each target of Experiment 2 in the invisible-cursor condition. (The closed circles are the data, and the solid line is the best fitting linear regression function.) 


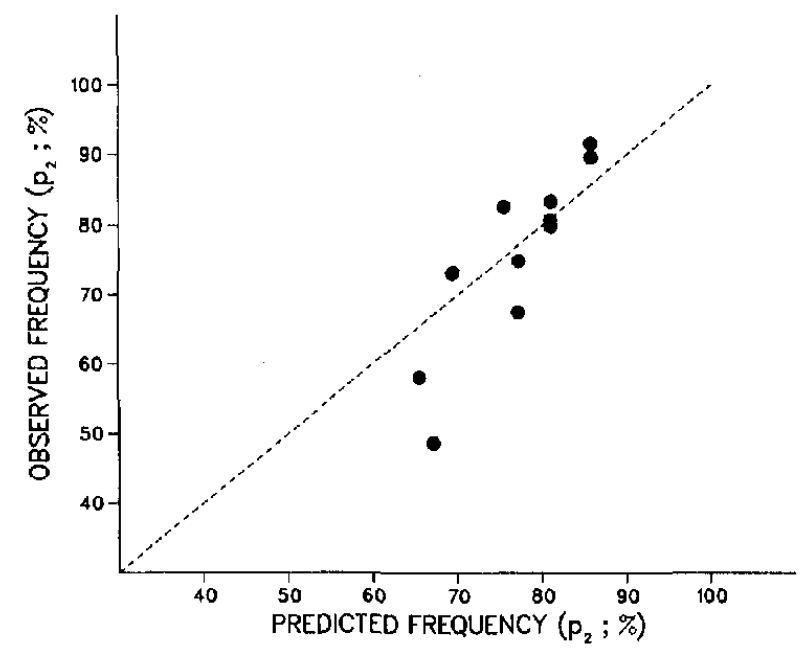

Figure 18. Relative frequencies of secondary submovements $\left(p_{2}\right)$ in the invisible-cursor condition of Experiment 2. (The closed circles represent pairs of observed and predicted values for each target, and the dashed diagonal line represents the ideal function that would apply if the predictions of the stochastic optimized-submovement model fit the observations perfectly.)

$35 \sqrt{D / W_{g}}$; for the visible-cursor condition, $T_{2}=-29+$ $71 \sqrt{D / W_{\mathrm{s}}}$ ). Eliminating the cursor increased the intercept and decreased the slope ( $p<.05$ in each case).

This pattern of results may be summarized in terms of the relative magnitudes for the mean secondary-submovement durations under the two cursor conditions. When target difficulty was low $\left(\sqrt{D / W_{\mathrm{s}}}<2.5\right), T_{2}$ was about $15 \mathrm{~ms}$ greater on the average with the cursor invisible than with the cursor visible (cf. Tables 5 and 6$)$. When target difficulty was high $\left(\sqrt{D / W_{\mathrm{s}}}>2.5\right)$, $T_{2}$ was about $50 \mathrm{~ms}$ less on the average with the cursor invisible than with the cursor visible. Thus, in essence, visual-feedback deprivation attenuated the effect of $\sqrt{D / W_{\mathrm{s}}}$ on $T_{2}$; subjects took less account of target difficulty during secondary submovements when they could not see the cursor.

Standard deviations of secondary-submovement endpoints. Associated with these effects of visual-feedback deprivation, there were marked changes in the standard deviations of secondary-submovement endpoints. $S_{2}$ was significantly greater under the invisible-cursor condition than under the visible-cursor condition $(p<.05)$. This can be seen in Figure 11, which shows $S_{2}$ versus the objective target width when the cursor was invisible (open circles and dashed line) and when it was visible (closed circles and solid line). Subjects' secondary submovements were not as precise without the cursor, and their spatial precision did not vary much as a function of the objective target width (Figure 11 , dashed line; $S_{2}=1.77-.021 \mathrm{~W} ; r=-.24$ ).

We may interpret the effects of visual-feedback deprivation on $S_{2}$ in either of two ways. One possibility is that eliminating the cursor increased the subjective target widths $\left(W_{s}\right)$, leading subjects to disperse their secondary submovements more broadly than when they could see the cursor. However, there is some evidence against this interpretation. When square-root functions were fit to the movement-time data (i.e., $T_{1}, T_{2}$, and $T$ vs. $\left.\sqrt{D / W_{\mathrm{s}}}\right)$ for the invisible-cursor condition, the obtained correlation was much higher if we based the fit on the originally estimated values of $W_{s}$ from the visible-cursor condition (Table 5) than if we based it on new $W_{\mathrm{s}}$ estimates made directly from data in the invisible-cursor condition $(r>.85$ vs. $r<.75)$. Given this outcome, it seems more likely that the values of $W_{\mathrm{s}}$ remained at least roughly the same across the two cursor conditions and that the effects of visual-feedback deprivation on $S_{2}$, $T_{2}$, and related dependent variables stemmed from other sources.

A second, more likely possibility is that visual-feedback deprivation affected secondary submovements by increasing subjects' spatial uncertainty about the endpoints of their primary submovements. If subjects had to guess where the inaccurate primary submovements ended when the cursor was invisible, this may have added extra variance to the secondary submovements, thereby increasing $S_{2}$. It would also account for increases observed in the error rates associated with visual-feedback deprivation.

Error rates. The error rates under the invisible-cursor condition were much higher on the average than those under the visible-cursor condition ( $44 \%$ vs. $10 \%)$. This difference was especially marked for the relatively difficult targets (cf. Tables 5 and 6). Such an outcome would be expected, given that the standard deviations of secondary-submovement endpoints $\left(S_{2}\right)$ were also large and rather insensitive to changes in the objective target width (Figure 11, open circles and dashed line).

To account precisely for the changes in error rates caused by visual-feedback deprivation, we must supplement the stochastic optimized-submovement model with some additional assumptions. These assumptions are necessary because the simplest version of the model does not specify exactly how subjects might make their secondary submovements under the invisiblecursor condition. The following treatment is therefore only tentative, and other assumptions could be tried instead. Nevertheless, the obtained results do illustrate the model's potential for further elaboration.

Our account of the error rates under the invisible-cursor condition assumes that when subjects cannot see the cursor, they are uncertain about the spatial positions of primary-submovement endpoints. According to this view, subjects make guesses about where their inaccurate primary submovements have ended, and on the basis of these guesses, they produce attempted corrective secondary submovements as if the cursor were visible. The contribution of the guessing process to the secondary submovements may be expressed by

$$
\left(S_{2 \mathrm{IVC}}\right)^{2}=\left(S_{2 \mathrm{VC}}\right)^{2}+U^{2},
$$

where $S_{2 \mathrm{IVC}}$ is the standard deviation of secondary-submovement endpoints under the invisible-cursor condition, $S_{2 \mathrm{vC}}$ is the corresponding standard deviation of secondary-submovement endpoints under the visible-cursor condition, and $U$ is the standard deviation of a random variable due to subjects' uncertainty regarding the spatial positions of the primary-submovement endpoints with an invisible cursor. We assume that $U$ is a linear function of $S_{1}$, the standard deviation of primary-submovement endpoints.

Using these additional assumptions, we have fit the stochastic optimized-submovement model to the error rates under the invisible-cursor condition. The fitting procedure incorporated terms for the primary submovements (i.e., $W_{\mathbf{s}}, T_{1}, S_{1}$, etc.) 


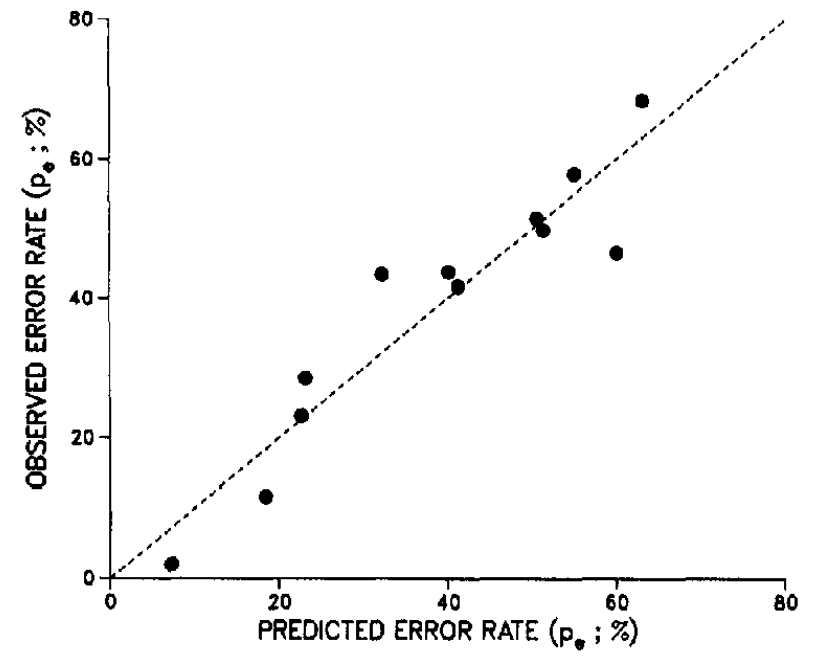

Figure 19. Observed versus predicted error rates (closed circles) for each target in the invisible-cursor condition of Experiment 2. (The dashed diagonal line represents the ideal function that would apply if the predictions of the stochastic optimized-submovement model fit the observations perfectly.)

equal to those found under the visible-cursor condition; only characteristics of the secondary submovements were assumed to change because of feedback deprivation, as outlined earlier. This yielded two types of results: a set of predicted versus observed error rates $\left(p_{e}\right)$ as a function of target condition (Figure 19 ) and an estimated linear relation between $U$ and $S_{1}$ (viz. $\left.U=.31+.77 S_{1}\right)$ that maximized the model's goodness of fit. The goodness of fit was not perfect; statistically significant deviations occurred between the observed and predicted values of $p_{\mathrm{e}}, \chi^{2}(12)=38.0, p<.01$. As Figure 19 shows, however, these values had a high positive correlation $(r=.94)$; most of the apparent deviations were less than $10 \%$ in absolute magnitude. So the model provided a reasonable first approximation when the cursor was invisible as well as when it was visible (cf. Figure 15).

\section{Discussion}

The results from the visible-cursor condition of Experiment 2 replicate those obtained in Experiment 1. Subjects' performance adhered closely to predictions based on the stochastic optimized-submovement model. Average total movement times $(T)$, mean primary-submovement durations $\left(T_{1}\right)$, standard deviations of primary-submovement endpoints $\left(S_{1}\right)$, relative frequencies of secondary submovements $\left(p_{2}\right)$, mean secondarysubmovement durations $\left(T_{2}\right)$, and error rates $\left(p_{\mathrm{e}}\right)$ again all fit qualitatively with the model. Most of these dependent variables also supported it quantitatively. Mixing the invisible-cursor condition with the visible-cursor condition did not markedly change the movement strategy that subjects adopted when visual feedback was available.

The results from the invisible-cursor condition provide new information about how subjects respond to visual-feedback deprivation during rapid spatially constrained movements. In presenting the rationale for Experiment 2, we outlined two possible movement strategies that could be adopted when visual feedback is absent. One strategy would entail producing slower and more accurate primary submovements, thereby eliminating a need for secondary movements while maintaining a low error rate at the expense of increased average total movement times. The other strategy, based on the stochastic optimizedsubmovement model, would entail using the same sort of primary-secondary submovement combinations as when visual feedback is available. This latter alternative has the advantage of maintaining relatively low average total movement times, but it may yield significantly increased error rates when subjects are deprived of visual feedback, assuming that accurate secondary submovements require high-quality input about the outcomes of primary submovements. We now have grounds to determine which of these two strategies was actually used by subjects here.

When subjects could not see the cursor, there were no significant increases in either the mean primary-submovement durations, the mean secondary-submovement durations, or the average total movement times compared with what happened under the visible-cursor condition. Instead, two of these variables (i.e., $T_{2}$ and $T$ ) decreased slightly, whereas the other $\left(T_{1}\right)$ remained about the same, and they were all characterized by square-root trade-off functions of $D / W_{\mathrm{s}}$ (cf. Figures 12 and 16). This rejects the one-submovement strategy and supports the two-submovement strategy as an account of performance for the invisiblecursor condition. It also provides more support for the tenets of the stochastic optimized-submovement model, confirming subjects' strong inclination toward movement-time minimization.

A persistent use of the two-submovement strategy is likewise suggested by other aspects of the results from the invisible-cursor condition. The relative frequencies of secondary submovements did not change much when concurrent visual feedback was absent (cf. Figures 14 and 18), but the error rates increased dramatically (cf. Figures 15 and 19). This accords with the model's assumption that accurate secondary submovements depend on reliable feedback regarding the outcomes of primary submovements. Subjects' desire to maintain low error rates did not outweigh their propensity to minimize average total movement times. Our findings therefore confirm and extend those of previous investigators who have reported that movement times approximate Fitts' law even under conditions of visual-feedback deprivation (Crossman \& Goodeve, 1963/1983; Prablanc et al., 1979; Wallace \& Newell, 1983).

The data from the invisible-cursor condition also help delineate exactly how the mechanisms used to prepare and produce secondary submovements work under the visible-cursor condition. In principle, secondary submovements need not stem from a feedback-based mechanism (Keele, 1981). They could be programmed as part of a submovement series before action is initiated, and they might then follow a predetermined course regardless of the outcomes achieved by primary submovements. Or secondary submovements could reflect the passive mechanical consequences of a mismatch between outputs by a phasic "pulse" mechanism and a tonic "step" mechanism in a mass-spring movement system (Bahill, Clark, \& Stark, 1975). Given the results of Experiment 2, however, these alternatives do not seem to be probable sources of the secondary submovements observed when visual feedback was available. If they had been, then eliminating the cursor should not have increased subjects' error rates, because the mechanisms just described could continue to operate as well even without such feedback. Instead, it seems more likely that the secondary submovements 
under the visible-cursor condition stemmed from active processing of visual feedback.

As suggested by previous investigators (e.g., Crossman \& Goodeve, 1963/1983), production of secondary submovements under the invisible-cursor condition may have been mediated by kinesthetic feedback. Correction mechanisms that use such feedback could account for error rates being greater when the cursor was invisible than when it was visible. We depicted target distance and width abstractly on the display screen relative to the actual position and kinematics of the moving wrist, so the required mapping to proprioceptive and kinesthetic variables was not as straightforward as in stylus tapping or arm pointing, where subjects look directly at their movements (cf. Prablanc et al., 1979; Wallace \& Newell, 1983). This perhaps precluded subjects from having sufficiently precise information about the spatial positions of their primary-submovement endpoints under the invisible-cursor condition, thereby leading to increased standard deviations of secondary-submovement endpoints (Equation 10) and decreased accuracy.

With these possibilities in mind, it is likewise of interest to note that eliminating concurrent visual feedback in Experiment 2 did not markedly change the relation between the standard deviations of the primary-submovement endpoints $\left(S_{1}\right)$ and the average velocities of the primary submovements $\left(D_{1} /\right.$ $T_{\mathrm{l}}$ ). Even though the secondary-submovement endpoints had much greater standard deviations $\left(S_{2}\right)$ under the invisible-cursor condition (Figure 11), the slope and intercept of $S_{1}$ versus $D_{1} / T_{1}$ for this condition were fairly similar to those under the visible-cursor condition (cf. Figures 13 and 17). This result would be expected if, as the stochastic optimized-submovement model assumes, neuromotor noise arises in mechanisms separate from those that mediated the feedback-deprivation effects.

\section{General Discussion}

The success of the stochastic optimized-submovement model strengthens the foundation for a unified theory of rapid aimed movements. As outlined at the beginning of this article, the model's premises are predicated partly on the linear speed-accuracy trade-off found previously during temporally constrained movement tasks (Schmidt et al., 1978, 1979; Wright, 1983a, 1983b; Wright \& Meyer, 1983; Zelaznik et al., 1981, in press). We have argued that the linear trade-off and the stochastic mechanisms underlying it constitute basic psychophysical characteristics of biological motor systems. These characteristics place fundamental limitations on how people may perform other types of movement tasks. Attempts to cope optimally with such limitations may lead to a logarithmic speed-accuracy trade-off (Fitts' law) during the performance of spatially constrained movement tasks. The latter type of task, which imposes a relatively lax temporal constraint, allows subjects to organize a series of optimal submovements that minimize average total movement times despite the random variability caused by neuromotor noise. It is this submovement organization that mediates the logarithmic trade-off as well as other salient features of spatially constrained movements, including distributions of submovement endpoints, relative frequencies of secondary submovements, and so forth. Separate sets of principles are not required to explain the performance of different tasks involving alternative speed-accuracy trade-offs for rapid aimed move- ments (cf. Hancock \& Newell, 1985; Schmidt et al., 1978, 1979).

From the perspective of the stochastic optimized-submovement model, it is also clearer now why Fitts' law has been such an ubiquitous phenomenon in the literature on human motor performance. People presumably prefer to perform well at spatially constrained movement tasks despite individual ideosyncracies and ancillary differences across environmental situations. Their movements are also presumably influenced by neuromotor noise regardless of the particular context in which they take place. These considerations together with the present theoretical framework rationalize previous reports that (a) Fitts' law applies not only to stylus tapping but also to wrist rotations (Crossman \& Goodeve, 1963/1983), microscopic finger manipulations (Langolf et al., 1976), arm extensions (Kerr \& Langolf, 1977), and other physical actions; (b) Fitts' law applies to movements made not only in laboratory experiments but also under water (Kerr, 1973), in industrial settings (Barnes, 1963), and elsewhere; (c) Fitts' law applies not only to normal young adults but also to the elderly (Welford, 1968), patients with Parkinson's disease (Flowers, 1976), and mental retardates (Wade et al., 1978).

\section{Links With Allied Topics}

Sensory and perceptual processes. The stochastic optimized-submovement model bears a close relation to some viewpoints regarding human perception and sensory psychophysics. In psychophysical research, it has been hypothesized that the performance of subjects on signal-detection and stimulus-discrimination tasks approximates the behavior of an ideal observer who must cope with internal noise (Green \& Swets, 1966). Important insights about sensory processes have emerged from attempts to assess the quality of this approximation in terms of receiver operating-characteristic (ROC) curves, measures of perceptual sensitivity $\left(d^{\prime}\right)$, and response bias $(\beta)$. Analogously, our findings demonstrate that useful insights about movement production may be reached by comparing subjects' performance with that of an ideal actor who, like the ideal observer, must cope with internal noise in the face of specified task demands.

Prosthetics and robotics. The theoretical ideas outlined here are also relevant for research on artificial as well as natural motor systems. Early prototypes of mechanical prostheses and robots were designed as relatively rigid noise-free devices, without the inherent flexibility or random variability common to the neuromotor systems of natural organisms. Recent developments on these fronts have, however, witnessed a growing concern over making artificial motor systems more intelligent and lifelike (e.g., Benati, Gaglio, Morasso, Tagliasco, \& Zaccaria, 1980; Hollerbach, 1982). This concern has been stimulated by a desire to achieve devices with greater adaptability. There has also been a growing attempt to identify principles of movement control embodied in natural motor systems that may help make artificial systems more economical and efficient (e.g., Nelson, 1983). As an example of how to implement ideal time-optimal performance under noisy circumstances, perhaps the stochastic optimized-submovement model will contribute in the future to these efforts. It could turn out, for example, that noisiness is a necessary feature of any truly adaptive motor system (Holland 
Table 7

Observed Versus Predicted $T_{2} / T$ Ratios in Experiments 1 and 2 for Each Target Condition

\begin{tabular}{|c|c|c|c|c|c|c|c|}
\hline \multirow[b]{2}{*}{$\begin{array}{c}\text { Width } \\
\text { (degree) }\end{array}$} & \multirow[b]{2}{*}{$\begin{array}{l}\text { Distance } \\
\text { (degree) }\end{array}$} & \multicolumn{3}{|c|}{ Observed ratio $\left(T_{1} / T\right)$} & \multicolumn{3}{|c|}{ Predicted ratio $\left(T_{1} / T\right)$} \\
\hline & & Experiment 1 & $\begin{array}{l}\text { Experiment } 2 \\
(V C)\end{array}$ & $\begin{array}{l}\text { Experiment } 2 \\
(I V C)\end{array}$ & Experiment 1 & $\begin{array}{l}\text { Experiment } 2 \\
(V C)\end{array}$ & $\begin{array}{c}\text { Experiment } 2 \\
(I V C)\end{array}$ \\
\hline 1.61 & $\begin{array}{l}15.81 \\
25.00\end{array}$ & $\begin{array}{l}.507 \\
.530\end{array}$ & $\begin{array}{l}.597 \\
.504\end{array}$ & $\begin{array}{l}.614 \\
.623\end{array}$ & $\begin{array}{l}.525 \\
.513\end{array}$ & $\begin{array}{l}.520 \\
.515\end{array}$ & $\begin{array}{l}.519 \\
.521\end{array}$ \\
\hline 2.54 & $\begin{array}{l}10.00 \\
15.81 \\
25.00 \\
39.52\end{array}$ & $\begin{array}{l}.699 \\
.628 \\
.605 \\
.528\end{array}$ & $\begin{array}{l}.741 \\
.602 \\
.533 \\
.503\end{array}$ & $\begin{array}{l}.695 \\
.647 \\
.599 \\
.570\end{array}$ & $\begin{array}{l}.555 \\
.532 \\
.520 \\
.513\end{array}$ & $\begin{array}{l}.540 \\
.520 \\
.515 \\
.508\end{array}$ & $\begin{array}{l}.546 \\
.525 \\
.523 \\
.510\end{array}$ \\
\hline 4.01 & $\begin{array}{l}10.00 \\
15.81 \\
25.00 \\
39.52\end{array}$ & $\begin{array}{l}.750 \\
.642 \\
.626 \\
.594\end{array}$ & $\begin{array}{l}.636 \\
.626 \\
.530 \\
.496\end{array}$ & $\begin{array}{l}.672 \\
.553 \\
.607 \\
.576\end{array}$ & $\begin{array}{l}.555 \\
.542 \\
.522 \\
.516\end{array}$ & $\begin{array}{l}.544 \\
.523 \\
.515 \\
.508\end{array}$ & $\begin{array}{l}.531 \\
.529 \\
.525 \\
.520\end{array}$ \\
\hline 6.34 & $\begin{array}{l}15.81 \\
25.00\end{array}$ & $\begin{array}{l}.714 \\
.653\end{array}$ & $\begin{array}{l}.634 \\
.562\end{array}$ & $\begin{array}{l}.620 \\
.578\end{array}$ & $\begin{array}{l}.559 \\
.536\end{array}$ & $\begin{array}{l}.536 \\
.523\end{array}$ & $\begin{array}{l}.520 \\
.527\end{array}$ \\
\hline
\end{tabular}

Note. $V C=$ visible-cursor condition; $I V C=$ invisible-cursor condition; $T_{1}=$ mean primary-submovement duration; $T=$ average total movement time.

et al., 1986) and that such a system would benefit significantly from incorporating at least part of the present model's optimization scheme.

\section{Caveats About the Model}

Of course, the current version of the stochastic optimizedsubmovement model is not the final word on rapid spatially constrained movements. To derive precise quantitative predictions, we have made several simplifying assumptions, which may require additional scrutiny and modification. Our assumptions include the following: (a) Subjects' movements to hit a target region include only one or two voluntary submavements; (b) the spatial endpoints of the primary and secondary submovements have normal distributions whose means are located at the center of the target region; and (c) the average velocity of the primary submovements is programmed to minimize average total movement times as a function of target distance, target width, and associated neuromotor noise, yielding perfect timeoptimal performance. Empirical justification for each assumption has been given on the basis of our experiments and those of past investigators.

Nevertheless, certain aspects of the stochastic optimized-submovement model still remain open to debate. As mentioned already, some investigators have reported observing more than two submovements per trial (e.g., Crossman \& Goodeve, 1963/ 1983; Jagacinski et al., 1980), contrary to the model's two-submovement assumption. Evidence has also been found that submovement endpoints are not always distributed symmetrically about the center of the target region. On occasion, more undershoots than overshoots of the target region have occurred (e.g., Fitts, 1954, Experiment 1, 1-lb stylus condition), suggesting a bias toward systematically short submovements, with which the model has yet to deal. Similarly, submovement durations may not always vary as the model predicts. For example, Annett et al. (1958) observed primary submovements whose mean durations were essentially independent of target distance and width, rather than being directly related to the distance-width ratio.

\section{Suboptimality of Movement Production}

These considerations serve to reiterate questions about the degree to which human motor performance is truly ideal. In particular, are rapid spatially constrained movements produced as optimally as they might be? Although we have presented evidence supporting the stochastic optimized-submovement model, we do not yet wish to answer this question with an unqualified affirmative. Some other facets of our data suggest that subjects do not always produce their movements in an entirely optimal fashion.

Secondary-submovement frequencies. For example, consider the relative frequencies of secondary submovements found in Experiments 1 and 2 . The results there revealed small but statistically significant deviations between the observed values of $p_{2}$ and the predictions of the stochastic optimized-submovement model (Figures 9, 14, and 18). Secondary submovements occurred less frequently than predicted for easy targets and more frequently than predicted for difficult targets.

These deviations may have happened because subjects acted more or less conservatively than the ideal in programming and executing their primary submovements. Perhaps primary submovements for the difficult targets were programmed to be too fast. Such faster-than-ideal primary submovements would suffer from excessively high neuromotor noise (Equation 2), leading to excessively frequent target misses, and so they would require more secondary corrective submovements than necessary. On the other hand, subjects may have programmed primary submovements for the easy targets to be too slow. Such slower-than-ideal primary submovements would experience relatively little effect of the noise, resulting in fewer target misses than allowed, and so they would fail to yield as many secondary submovements as expected.

Primary-submovement durations. The hypothesis that subjects sometimes act too conservatively in producing their primary submovements is supported further by a more detailed analysis of the primary-submovement durations. According to the stochastic optimized-submovement model, the ratio of the 
mean primary-submovement durations $\left(T_{1}\right)$ to the average total movement times $(T)$ should satisfy the following equation:

$$
\frac{T_{1}}{T}=\left[2-(1 / \theta)\left(W_{\mathrm{s}} / D\right)\right]^{-1}
$$

where $W_{\mathrm{s}} / D$ is the ratio of the subjective target width to distance, and $\theta$ is one of the parameters mentioned elsewhere (Footnote 12, Footnote 16, and Appendix). However, when we compared the observed $T_{1} / T$ ratios with the predicted ones, the fit was good only for the relatively difficult target conditions.

Table 7 shows the obtained results. For the easy target conditions, the observed $T_{1} / T$ ratios tended to be considerably larger than expected. In some cases, the primary submovements contributed between $60 \%$ and $75 \%$ of the average total movement time, whereas the stochastic optimized-submovement model predicts that the observed ratios should have been between $50 \%$ and $55 \%$. Subjects spent somewhat more time than ideal on their primary submovements, consistent with the pattern of conservatism discussed earlier. By making faster primary submovements for the easy targets, it might have been possible to perform better than we found here, even though this speedup would have entailed making more secondary submovements.

Sources of suboptimality. Several possible sources could contribute to the apparent suboptimality of performance revealed by Experiments 1 and 2 . The pattern of conservatism already discussed seems analogous to what other investigators have found regarding human inductive inference and statistical decision making. In some situations, people underestimate the true probabilities of likely events and overestimate the true probabilities of unlikely events (Tversky \& Kahneman, 1981). Such behavior does not conform exactly to the prescripts of Bayes' theorem, a normative rule that combines prior probabilities with sampled data to estimate unbiased posterior probabilities (Edwards et al., 1963). Similarly, if subjects tend to underestimate the high probability of hitting an easy target with a relatively fast primary submovement, and if they overestimate the low probability of hitting a difficult target with a relatively slow primary submovement, then this might bias them toward deviations of primary-submovement durations and secondary-submovement frequencies as we observed.

Suboptimality of performance in the time domain might also stem from other factors. Suppose subjects are concerned with minimizing additional independent variables besides just average total movement time. These variables could involve the amounts of effort and stress (e.g., jerk) experienced during movement production (Hogan, 1984; Nelson, 1983). If effort, stress, and so forth are taken into account along with temporal factors as part of an overall optimum movement-control strategy, then the prescriptions made by the stochastic optimizedsubmovement model would have to be modified accordingly, perhaps exhibiting a pattern of conservatism in the time, space, and submovement-frequency domains. Hopefully, we may address these considerations through appropriate extensions of the model without abandoning its essential spirit.

\section{Future Research Directions}

In conclusion, the stochastic optimized-submovement model opens a number of avenues for investigating the details of hu- man motor performance. Following our general discussion, it would be interesting to conduct studies on the effects of explicit movement-training techniques designed to promote the optimality of subjects' performance during spatially constrained movement tasks. The model's predictions (Table 1) could provide a useful benchmark against which to assess the efficacy of alternative instructional formats and practice protocols. By comparing these predictions with data collected under various real-world conditions, one may eventually achieve significant improvements of people's performance in practical situations requiring skilled movement.

\section{References}

Abrams, R. A., Kornblum, S., Meyer, D. E., \& Wright, C. E. (1983, November). Fitts' law: Optimization of initial ballistic impulses for aimed movements. Paper presented at the meeting of the Psychonomic Society, San Diego, CA.

Annett, J., Golby, C. W., \& Kay, H. (1958). The measurement of elements in an assembly task: The information output of the human motor system. Quarterly Journal of Experimental Psychology, 10, 111.

Bahill, A. T., Clark, M. R., \& Stark, L. (1975). Glissades-Eye movements generated by mismatched components of the saccadic motoneuronal control signal. Mathematical Biosciences, 26, 303-318.

Bailey, G. B., \& Presgrave, R. (1958). Basic motion time study. New York: McGraw-Hill.

Barnes, R. (1963). Motion and time study: Design and measurement of work. New York: Wiley.

Benati, M., Gaglio, S., Morasso, P., Tagliasco, V., \& Zaccaria, R. (1980). Anthropomorphic robotics (Pt. II). Analysis of manipulator dynamics and the output motor impedance. Biological Cybernetics, 38 , 141-150.

Bizzi, E., Dev, P., Morasso, P., \& Polit, A. (1978). Effect of load disturbances during centrally initiated movements. Journal of Neurophysiology, 41, 542-556.

Braine, M. D. S. (1978). On the relation between the natural logic of reasoning and standard logic. Psychological Review, 85, 1-21.

Carlton, L. G. (1979). Control processes in the production of discrete aiming responses. Journal of Human Movement Studies, 5. 115-124.

Carlton, L. G. (1980). Movement control characteristics of aiming responses. Ergonomics, 23, 1019-1032.

Carlton, L. G. (1981a). Processing visual feedback information for movement control. Journal of Experimental Psychology: Human Perception and Performance, 7, 1019-1030.

Carlton, L. G. (1981b). Visual information: The control of aiming movements. Quarterly Journal of Experimental Psychology, 33A, 8793.

Carlton, L. G., \& Newell, K. M. (1988). Force variability and movement accuracy in space-time. Journal of Experimental Psychology: Human Perception and Performance, 14, 24-36.

Crossman, E. R. F. W., \& Goodeve, P. J. (1983). Feedback control of hand-movement and Fitts' law. Quarterly Journal of Experimental Psychology, 35A, 251-278. Original work presented at the meeting of the Experimental Psychology Society, Oxford, England, July 1963.

Edwards, W. (1961). Behavioral decision theory. Annual Review of Psychology, 12, 473-498.

Edwards, W., Lindman, H., \& Savage, L. J. (1963). Bayesian statistical inference for psychological research. Psychological Review, 70, 193242.

Fel'dman, A. G. (1966). Functional maintenance of a steady posture: II. Controllable parameters of the muscles. Biophysics, 11, 565-578.

Fitts, P. M. (1954). The information capacity of the human motor system in controlling the amplitude of movement. Journal of Experimental Psychology, 47, 381-391. 
Fitts, P. M., \& Peterson, J. R. (1964). Information capacity of discrete motor responses. Journal of Experimental Psychology, 67, 103-112.

Fitts, P. M., \& Radford, B. K. (1966). Information capacity of discrete motor responses under different cognitive sets. Journal of Experimental Psychology, 71, 475-482.

Flowers, K. A. (1976). Visual "closed loop" and "open loop" characteristics of voluntary movement in patients with Parkinsonism and intention tremor. Brain, 99, 269-310.

Green, D. M., \& Swets, J. A. (1966). Signal detection theory and psychophysics. New York: Wiley.

Hancock, P. A., \& Newell, K. M. (1985). The movement speed-accuracy relationship in space-time. In H. Heuer, U. Kleinbeck, \& K. H. Schmidt (Eds.), Motor behavior: Programming, control, and acquisition (pp. 153-188). New York: Springer-Verlag.

Hogan, N. (1984). An organizing principle for a class of voluntary movements. The Journal of Neuroscience, 4, 2745-2754.

Holland, J. H., Holyoak, K. J., Nisbett, R. E., \& Thagard, P. R. (1986). Induction. Processes of inference, learning, and discovery. Cambridge: MIT Press.

Hollerbach, J. M. (1982). Computers, brains and the control of movement. Trends in Neurosciences, 5, 189-192.

Howarth, C. I., Beggs, W. D. A., \& Bowden, J. M. (1971). The relationship between speed and accuracy of movement aimed at a target. Acta Psychologica, 35, 207-218.

Jagacinski, R. J., Repperger, D. W., Moran, M. S., Ward, S. L., \& Glass, B. (1980). Fitts' law and the microstructure of rapid discrete movements. Journal of Experimental Psychology: Human Perception and Performance, 6, 309-320.

Kahneman, D., \& Tversky, A. (1973). On the psychology of prediction. Psychological Review, 80, 237-251.

Kaiser, J. F., \& Reed, W. A. (1977). Data smoothing using low-pass digital filters. Review of Scientific Instruments, 48, 1447-1456.

Keele, S. W. (1968). Movement control in skilled motor performance. Psychological Bulletin, 70, 387-403.

Keele, S. W. (1981). Behavioral analysis of movement. In V. Brooks (Ed.), Handbook of physiology: The nervous system, Vol. 2. Motor control (pp. 1391-1414). Baltimore, MD: American Physiological Society.

Keele, S. W., \& Posner, M. I. (1968). Processing of visual feedback in rapid movements. Journal of Experimental Psychology. 77, 155-158.

Kerr, B. A., \& Langolf, G. D. (1977). Speed of aiming movements. Quarterly Journal of Experimental Psychology, 29, 475-481.

Kerr, R. (1973). Movement time in an underwater environment. Journal of Motor Behavior, 5, 175-178.

Klapp, S. T. (1975). Feedback versus motor programming in the control of aimed movements. Journal of Experimental Psychology: Human Perception and Performance, 104, 147-153.

Kleinman, D. L., Baron, S., \& Levison, W. H. (1970). An optimal control model of human response: Part I. Theory and validation. Automatica, 6, 357-369.

Kvalseth, T. O. (1980). An alternative to Fitts' law. Bulletin of the Psychonomic Society, 16. 371-373.

Langolf, G. D., Chaffin, D. B., \& Foulke, J. A. (1976). An investigation of Fitts' law using a wide range of movement amplitudes. Journal of Motor Behavior, 8, 113-128.

Legge, D., \& Barber, P. J. (1976). Information and skill. London: Methuen.

Meyer, D. E., Smith, J. E. K., \& Wright, C. E. (1982). Models for the speed and accuracy of aimed movements. Psychological Review, 89 , 449-482.

Miller, G. A. (1953). What is information measurement? American $P_{s y}$ chologist, 8, 3-11.

Nelson, W. (1983). Physical principles for economies of skilled movements. Biological Cybernetics, 46, 135-147.

Nisbett, R., \& Ross, L. (1980). Human inference: Strategies and shortcomings of social judgment. Englewood Cliffs, NJ: Prentice-Hall.
Osherson, D. (1975). Logic and logical models of thinking. In R. Falmagne (Ed.), Reasoning: Representation and process (pp. 81-91). New York: Wiley.

Partridge, L. D. (1967). Signal handling characteristics of load-moving skeletal muscle. American Journal of Physiology, 7, 853-861.

Poulton, E. C. (1974). Tracking skill and manual performance. New York: Academic Press.

Prablanc, C., Echallier, J. F., Komilis, E., \& Jeannerod, M. (1979). Optimal response of eye and hand motor systems in pointing at a visual target: I. Biological Cybernetics, 35, 113-124.

Sakitt, B. (1980). A spring model and equivalent neural network for arm posture control. Biological Cybernetics, 37, 227-234.

Schmidt, R. A., Sherwood, D., Zelaznik, H. N., \& Leikind, B. (1985). Speed-accuracy tradeoffs in motor behavior: Theories of impulse variability. In H. Heuer, U. Kleinbeck, \& K. H. Schmidt (Eds.), Motor behavior: Programming, control, and acquisition (pp. 79-123). New York: Springer-Verlag.

Schmidt, R. A., Zelaznik, H. N., \& Frank, J. S. (1978). Sources of inaccuracy in rapid movement. In G. E. Stelmach (Ed.), Information processing in motor control and learning (pp. 183-203). New York: Academic Press.

Schmidt, R. A., Zelaznik, H., Hawkins, B., Frank, J. S., \& Quinn, J. T., Jr. (1979). Motor-output variability: A theory for the accuracy of rapid motor acts. Psychological Review, 86, 415-451.

Shannon, C., \& Weaver, W. (1949). The mathematical theory of communication. Urbana: University of Illinois Press.

Sheridan, M. R. (1979). A reappraisal of Fitts' law. Journal of Motor Behavior, 11, 179-188.

Simon, H. A. (1957). Models of man. New York: Wiley.

Stein, R. B., \& Lee, R. G. (1981). Tremor and clonus. In V. Brooks (Ed.), Handbook of physiology: The nervous system, Vol. 2. Motor control (pp. 325-344). Baltimore, MD: American Physiological Society.

Tversky, A., \& Kahneman, D. (1974). Judgment under uncertainty: Heuristics and biases. Science, 185, 1124-1131.

Tversky, A., \& Kahneman, D. (1981). The framing of decisions and the psychology of choice. Science, 211, 453-458.

Vince, M. A. (1948). Corrective movements in a pursuit task. Quarterly Journal of Experimental Psychology, 1, 85-103.

von Holst, E. (1954). Relations between the central nervous system and the peripheral organs. Animal Behaviour, 2, 89-94.

Wade, M. G., Newell, K. M., \& Wallace, S. A. (1978). Decision time and movement time as a function of response complexity in retarded persons. American Journal of Mental Deficiency, 83, 135-144.

Wallace, S. A. (1981). An impulse-timing theory for reciprocal control of muscular activity in rapid, discrete movements. Journal of Motor Behavior, 13, 144-160.

Wallace, S. A., \& Newell, K. M. (1983). Visual control of discrete aiming movements. Quarterly Journal of Experimental Psychology, 35A, $311-321$.

Welford, A. T. (1968). Fundamentals of skill. London: Methuen.

Winer, B. J. (1962). Statistical principles in experimental design. New York: McGraw-Hill.

Woodworth, R. S. (1899). The accuracy of voluntary movement. Psychological Review, 3(2, Whole No. 13).

Woodworth, R. S. (1938). Experimental psychology. New York: Holt.

Wright, C. E. (1983a). Spatial and temporal variability of aimed movements with three contrasting goal points. Unpublished doctoral dissertation, University of Michigan, Ann Arbor.

Wright, C. E. (1983b, November). Spatial variability of movements to three contrasting goal points. Paper presented at the meeting of the Psychonomic Society, San Diego, CA.

Wright, C. E., \& Meyer, D. E. (1983). Conditions for a linear speedaccuracy trade-off in aimed movements. Quarterly Journal of Experimental Psychology, 35A, 279-296. 
Zelaznik, H. N., Hawkins, B., \& Kisselburgh, L. (1983). Rapid visual feedback processing in single-aiming movements. Journal of Motor Behavior, 15, 217-236.

Zelaznik, H. N., Mone, S., McCabe, G. P., \& Thaman, C. (in press). Role of temporal and spatial precision in determining the nature of the speed-accuracy trade-off in aimed-hand movements. Journal of Experimental Psychology: Human Perception and Performance.

Zelanik, H. N., \& Schmidt, R. A. (1983, November). Kinematic proper- ties of single-aiming movements. Paper presented at the meeting of the Psychonomic Society, San Diego, CA.

Zelaznik, H. N., Schmidt, R. A., \& Gielen, S. C. A. M. (1986). Kinematic properties of rapid aimed hand movements. Journal of Motor Behavior, 18, 353-372.

Zelaznik, H. N., Shapiro, D. C., \& McColsky, D. (1981). Effects of a secondary task on the accuracy of single aiming movements. Journal of Experimental Psychology: Human Perception and Performance, 7 , 1007-1018.

\section{Appendix}

This Appendix demonstrates that the stochastic optimized-submovement model yields the quantitative predictions outlined in the text (Equations 4 through 9). To facilitate the demonstration, we rely on several assumptions introduced and justified earlier (see Basic Assumptions).

The model's assumption of two submovements lets us represent the average total time $(T)$ by the following equation:

$$
T=T_{1}+T_{2}
$$

where $T_{1}$ and $T_{2}$ denote the mean durations of primary and secondary submovements, respectively. From Equation 2 of the text, it is given that

$$
T_{1}=\frac{K D_{1}}{S_{1}},
$$

where $D_{1}$ is the mean distance traveled by the primary submovements, and $S_{1}$ is the standard deviation of the primary-submovement endpoints. Also, from previous assumptions, $D_{1}=D$, where $D$ is the distance from the home position to the center of the target region. Thus, we may rewrite Equation $\mathrm{A} 1$ as

$$
T=\frac{K D}{S_{1}}+T_{2} .
$$

Next suppose that a primary submovement ends $\Delta$ units from the center of the target region, and suppose that $T_{2 \Delta}$ is the mean duration of secondary submovements conditional on the value of $\Delta$. When $|\Delta|<$ $W / 2$, where $W$ is the target width, no secondary submovements are assumed to occur, and $T_{2 \Delta}=0$. When $|\Delta| \geq W / 2$, secondary submovements are assumed to occur, and $T_{2 \Delta}=K \Delta / S_{2}$ from Equation 3 of the text, where $S_{2}$ is the standard deviation of the secondary-submovement endpoints. Given that the secondary-submovement endpoints are also assumed to have a normal distribution such that a proportion $c_{2}$ of them are inside the target region, $S_{2}=W / 2 z_{\mathrm{c} 2}$, where $z_{\mathrm{c} 2}$ is a unit-normal deviate whose value yields $P\left(-z_{\mathrm{c} 2} \leq z \leq z_{\mathrm{c} 2}\right)=c_{2}$ for a standardized normal random variable $z$. Furthermore, we may integrate $T_{2 \Delta}$ throughout the range of possible $\Delta$ values, taking into account the probability that the primary-submovement endpoints are outside the target region. This yields Equation A4 for the unconditional mean duration of secondary submovements:

$$
\begin{array}{r}
T_{2}=\int_{-\infty}^{-W / 2} \frac{K \Delta}{S_{2}} n\left(\Delta \mid 0, S_{1}\right) d \Delta+\int_{W / 2}^{\infty} \frac{K \Delta}{S_{2}} n\left(\Delta \mid 0, S_{1}\right) d \Delta \\
=\frac{4 K z_{\mathrm{c} 2}}{W} \int_{W / 2}^{\infty} \Delta n\left(\Delta \mid 0, S_{1}\right) d \Delta,
\end{array}
$$

where $n\left(\Delta \mid 0, S_{1}\right)$ is the probability-density function of a normal random variable $\Delta$ with mean zero and standard deviation $S_{1}$ (i.e., $n\left(\Delta \mid 0, S_{1}\right)=$ $\left(S_{1} \sqrt{2 \pi}\right)^{-1} \exp \left(-\Delta^{2} / 2 S_{1}^{2}\right)$.
The last integral in Equation A4 may be evaluated by representing it in the form $\int e^{u} d u$, with $u=-\left(\Delta^{2} / 2 S_{1}{ }^{2}\right)$, and $d u=-\left(\Delta / S_{1}{ }^{2}\right) d \Delta$. This yields Equation $\mathrm{A} 5$ :

$$
T_{2}=\left(4 K S_{1} z_{\mathrm{c} 2} / W \sqrt{2 \pi}\right) \exp \left(-z_{\mathrm{c} 1}^{2} / 2\right),
$$

where $z_{\mathrm{cl}}$ is a unit-normal deviate such that $z_{\mathrm{cl}}=W / 2 S_{1}$ and $P\left(-z_{\mathrm{cl}} \leq\right.$ $\left.z \leq z_{\mathrm{e} 1}\right)=c_{1}$ for a standardized normal random variable $z$. As introduced earlier (see text), $c_{1}$ here denotes the probability that the normally distributed endpoints of the primary submovements fall within the target region of width $W$.

Equations $\mathrm{A} 3$ and $\mathrm{A} 5$ allow us to express $T$ in terms of $K, D, W, z_{\mathrm{cl}}$, and $z_{\mathrm{c} 2}$. Combining these equations with the preceding fact that $S_{1}=$ $W / 2 z_{\mathrm{cl}}$, we have

$$
T=\left(2 K D z_{\mathrm{c} 1} / W\right)+\left(2 K z_{\mathrm{c} 2} / z_{\mathrm{c} 1} \sqrt{2 \pi}\right) \exp \left(-z_{\mathrm{c} 1}^{2} / 2\right) .
$$

After $D$ and $W$ have been specified, the only free variable on the right side of Equation $A 6$ is $z_{\mathrm{cl}}$, assuming that the secondary submovements have a fixed probability $\left(c_{2}\right)$ of ending inside the target region. Thus, under the stochastic optimized-submovement model, minimizing the average total movement time, $T$, is equivalent to adjusting the primary submovements to have a standard deviation $S_{1}$ for which the corresponding $z_{c 1}$ minimizes $T$.

The optimal $S_{1}$ and $z_{\mathrm{cl}}$, together with the minimum $T$, may be calculated from Equation A6 by differentiating $T$ with respect to $z_{\mathrm{c} 1}$, setting the resultant derivative equal to zero, and then solving for $z_{\mathrm{c} 1}$. This sequence of steps yields

$$
\begin{aligned}
S_{1} & =.5 W \sqrt{\theta(D / W)-1} \\
z_{\mathrm{c} 1} & =1 / \sqrt{\theta(D / W)-1}, \\
\theta & =\left[\sqrt{2 \pi} \exp \left(z_{\mathrm{cl}}{ }^{2} / 2\right)\right] / z_{\mathrm{c} 2} .
\end{aligned}
$$

The parameter $\theta$ depends on the variable $z_{\mathrm{c} 1}$ (Equation A9), so Equation A8 may not be used to obtain $z_{\mathrm{c} 1}$ analytically. However, Equations $\mathrm{A} 8$ and $A 9$ may be used iteratively to obtain approximate numerical values for both $z_{\mathrm{c} 1}$ and $\theta$. One can initially set $z_{\mathrm{c} 1}$ equal to $\sqrt{W / D}$, substitute this value into the right side of Equation $\mathrm{A} 9$, and derive a value for $\theta$ on the left side. Next one can take the obtained $\theta$ value, substitute it into the right side of Equation $A 8$, derive a new value of $z_{\mathrm{c} 1}$, and then repeat the overall process iteratively until it converges. The ultimate value of $\theta$ will come very close to $\sqrt{2 \pi} / z_{\mathrm{c} 2}$ when $D / W$ is large, because $z_{\mathrm{c} 1}$ approaches zero and $\exp \left(z_{\mathrm{cl}}{ }^{2} / 2\right)$ approaches one as $D / W$ approaches infinity.

Substituting the right side of Equation A8 into Equation A6 and simplifying terms, we find that the minimum average total movement time is

$$
T=2 K(2 \theta \sqrt{D / W}-\sqrt{W / D) / \theta \sqrt{\theta-(W / D)}} .
$$

Furthermore, using Equations $\mathrm{A} 8$ and $\mathrm{A} 9$, it can be shown that 


$$
\begin{aligned}
& T_{1}=2 K \sqrt{D / W} / \sqrt{\theta-(W / D)}, \\
& T_{2}=2 K \sqrt{D / W} \sqrt{\theta-(W / D)} / \theta .
\end{aligned}
$$

As $D / W$ grows large, the term $W / D$ approaches zero, and the right sides of Equations A 10 through $A 12$ approach pure square-root functions of $D / W$, paralleling the claims made in the text.

The iterative solution for $z_{\mathrm{c} 1}$ in terms of Equations A8 and A9 will converge whenever $D / W z_{\mathrm{c} 2}>\sqrt{2 / \pi e}$. If $D / W z_{\mathrm{c} 2} \leq \sqrt{2 / \pi \mathrm{e}}$, then convergence will not occur. Instead, in the latter casc, a convergent iterative solution for $z_{\mathrm{cl}}$ can be obtained by selecting an initial approximate value of $z_{\mathrm{c} 1}$ and then applying the expression $z_{\mathrm{c} 1}=\left[2 \ln \left(1+z_{\mathrm{c} 1}{ }^{2}\right)-\right.$ $\left.2 \ln \left(D z_{\mathrm{c} 1} 2 \sqrt{2 \pi} / W z_{\mathrm{c}_{\hat{\mathrm{c}}}}\right)\right]^{.5}$ repeatedly. This follows from two related facts: (a) if $f(z)$ is a function such that $z^{*}=f\left(z^{*}\right)$, and if there exists a positive $\epsilon$ such that $\left|f^{\prime \prime}(z)\right|<1$ for all $z$ in the interval $\left(z^{*}-\epsilon, z^{*}+\epsilon\right)$, where $f^{\prime}(z)$ is the first derivative of $f(z)$, then the itcrative sequence $z_{n}=f\left(z_{n-1}\right), n=$ $2,3, \ldots$, will converge to $z^{*}$ whenever $\left|z_{1}-z^{*}\right|<\epsilon$; (b) if $z^{*}=f\left(z^{*}\right)$, but $\left|f^{\prime}(z)\right|>1$ for all $z$ in the interval $\left(z^{*}-\epsilon, z^{*}+\epsilon\right)$, then the iterative sequence $z_{\mathrm{n}}=f^{-1}\left(z_{n}\right), n=2,3, \ldots$, will converge to $z^{*}$, where $f^{-1}(z)$ is the inverse of $f(z)$.

Received September 9, 1985

Revision received September 17, 1987

Accepted September 23, 1987

\section{Members of Underrepresented Groups: Reviewers for Journal Manuscripts Wanted}

If you are interested in reviewing manuscripts for APA journals, the APA Publications and Communications Board would like to invite your participation. Manuscript reviewers are vital to the publication process. As a reviewer, you would gain valuable experience in publishing. The P \& C Board is particularly interested in encouraging members of underrepresented groups to participate more in this process.

If you are interested in reviewing manuscripts, please write to Leslie Cameron at the address below. Please note the following important points:

- To be selected as a reviewer, you must have published articles in peer-reviewed journals. The experience of publication provides a reviewer with the basis for preparing a thorough, objective evaluative review.

- To select the appropriate reviewers for each manuscript, the editor needs detailed information. Please include with your letter your vita. In your letter, please identify which APA journal you are interested in and describe your area of expertise. Be as specific as possible. For example, "social psychology" is not sufficient-you would need to specify "social cognition" or "attitude change" as well.

- Reviewing a manuscript takes time. If you are selected to review a manuscript, be prepared to invest the necessary time to evaluate the manuscript thoroughly.

Write to Leslie Cameron, Journals Office, APA, 1400 N. Uhle Street, Arlington, Virginia 22201. 